\title{
The syntax-semantics interface of 'respective' predication: A unified analysis in Hybrid Type-Logical Categorial Grammar
}

\author{
Yusuke Kubota \\ Robert Levine \\ University of Tsukuba \\ Ohio State University \\ Ohio State University
}

May 19, 2015

\begin{abstract}
This paper proposes a unified analysis of the 'respective' readings of plural and conjoined expressions, the internal readings of symmetrical predicates such as same and different, and the summative readings of expressions such as a total of \$10,000. These expressions pose significant challenges to compositional semantics, and have been studied extensively in the literature. However, almost all previous studies focus exclusively on one of these phenomena, and the close parallels and interactions that they exhibit have been mostly overlooked to date. We point out two key properties common to these phenomena: (i) they target all types of coordination, including nonconstituent coordination such as Right-Node Raising and Dependent Cluster Coordination; (ii) the three phenomena all exhibit multiple dependency, both by themselves and with respect to each other. These two parallels suggest that one and the same mechanism is at the core of their semantics. Building on this intuition, we propose a unified analysis of these phenomena, in which the meanings of expressions involving coordination are formally modelled as multisets, that is, sets that allow for duplicate occurrences of identical elements. The analysis is couched in Hybrid Type-Logical Categorial Grammar. The flexible syntax-semantics interface of this framework enables an analysis of 'respective' readings and related phenomena which, for the first time in the literature, yields a simple and principled solution for both the interactions with nonconstituent coordination and the multiple dependency noted above.
\end{abstract}

Keywords: 'respective' reading, symmetrical predicate, parasitic scope, hypothetical reasoning, coordination, Hybrid Type-Logical Categorial Grammar

\section{Introduction}

The so-called 'respective' readings of plural and conjoined expressions and the internal readings of symmetrical predicates such as same and different in $(1 \mathrm{a}, \mathrm{b})$ have posed difficult challenges to theories of the syntax-semantics interface (see Sect. 2 for the relevant literature). Summative predicates such as a total of $X$ in (1c) present a similar problem.

(1) a. John and Bill sang and danced, respectively. ('respective' reading) $=$ 'John sang and Bill danced'

b. $\{$ The same performer/Different performers $\}$ sang and danced.

(symmetrical predicate) $\approx$ 'The performer who sang and the performer who danced are the same/different' 
c. John and Bill spent a total of $\$ 10,000$ last year. (summative predicate) $=$ 'The amount that John spent last year and the amount that Bill spent last year add up to $\$ 10,000$ '

These phenomena interact with coordination, including nonconstituent coordination (NCC; both Right-Node Raising (RNR) and Dependent Cluster Coordination (DCC)):

(2) a. John read, and Bill reviewed, Barriers and $L G B$, (respectively).

('respective' reading, $\mathrm{RNR}$ )

b. John introduced the same girl to Chris on Thursday and (to) Peter on Friday.

(symmetrical predicate, DCC)

c. John spent, and Bill lost, a total of $\$ 10,000$ last year.

(summative predicates, RNR)

Moreover, these expressions can themselves be iterated and interact with one another to induce multiple dependencies:

(3) a. John and Bill introduced Mary and Sue to Chris and Pat (respectively).

b. John and Bill gave the same book to the same man.

c. John and Mary showed the same book to his brother and her sister (respectively).

d. John and Mary collected a total of $\$ 10,000$ for charity from his family and her clients, respectively.

e. John and Mary gave a total of $\$ 10,000$ to the same man.

Any adequate analysis of these phenomena needs to account for these facts. In particular, the parallel between the three phenomena in the multiple dependency cases in (3), especially, the interdependency between 'respective', symmetrical and summative predicates in (3c-e), raises the possibility that the same (or a similar) mechanism is at the core of the semantics of these phenomena.

The goal of the present paper is to propose precisely such a unified analysis of the three phenomena. While the 'respective' readings and symmetrical predicates have been extensively studied (separately) in the previous literature, there does not currently exist an analysis, at any level of formal explicitness, which offers a systematic explanation for their parallel behaviors observed above (though see Chaves (2012) for an important precursor). By building on several analyses from the previous literature, we develop an analysis that posits a common mechanism of pairwise predication for these phenomena, and show that this analysis straightforwardly captures their parallel behaviors.

\section{The meanings of 'respective', symmetrical and summative predicates}

\subsection{The empirical parallel between 'respective', symmetrical and summa- tive predicates}

'Respective' readings of plural and conjoined expressions (cf., e.g., Kay 1989; McCawley 1998; Gawron and Kehler 2004; Winter 1995; Bekki 2006; Chaves 2012) and the semantics of symmetrical predicates such as same and different (cf., e.g., Carlson 1987; Moltmann 1992; Beck 
2000; Barker 2007; Brasoveanu 2011) have been known to poses major challenges to theories of compositional semantics. Each of these two constructions alone present a set of quite complex problems of its own, and previous authors have thus mostly focused on studying the properties of one or the other. However, as we discuss below, the problems that the two phenomena exhibit are remarkably similar. A less frequently discussed type of sentences, but one which raises essentially the same problem for compositionality, comes from the interpretation of expressions such as a total of $X$ and $X$ in total. We call these expressions 'summative' predicates. Summative predicates have been discussed in the literature mostly in the context of Right-Node Raising (RNR) (Abbott 1976; Jackendoff 1977). Some representative examples of each construction were given in (1)-(3) in Sect. 1 above.

The difficulty that these phenomena pose can be illustrated by the following examples involving 'respective' readings: ${ }^{1}$

(4) a. John and Bill bought the book and the CD, respectively.

(NP coordination)

b. John and Bill ran and danced, respectively.

(VP coordination)

c. John read, and Bill listened to, the book and the CD, respectively.

(RNR)

d. John gave the book and the CD to Sue on Wednesday and to Mary on Thursday, respectively.

(DCC)

These examples exhibit readings that can be paraphrased by the sentences in (5). ${ }^{2,3}$

\footnotetext{
${ }^{1}$ It has been noted by Postal (1998), Kehler (2002) and Chaves (2012) that 'respective' readings interact with extraction, as exemplified by the following data (called 'interwoven dependency' by Postal (1998)):

(i) a. Which pilot ${ }_{i}$ and which sailor ${ }_{j}$ will Joan invite $\__{i}$ and Greta entertain $\__{j}$ respectively?

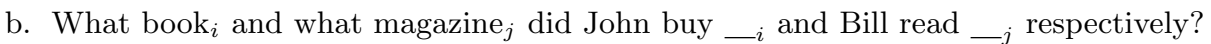

It is possible to construct parallel examples involving summative predicates (symmetrical predicates seem to be uncomfortable in fronted $w h$ or topicalized positions, and we weren't able to construct relevant examples):

(ii) How many frogs in total did Greg capture _ and Lucille train _ ?

The analysis we propose in Sect. 3 is in principle compatible with these data. However, since the analysis of ATB extraction in Hybrid TLCG is still under development (due to the fact that this type of extraction constitutes an (apparent) exception to the linearity of the calculus underlying TLCG in general, including our own version), we will not attempt to formulate an explicit analysis of these interactions in this paper.

${ }^{2}$ One might be inclined to think that the adjective respective in examples like the following should be given a parallel treatment:
}

(i) John and Bill talked to their respective supervisors.

However, as convincingly argued by Okada (1999) and Gawron and Kehler (2002), the properties of the adjective respective is significantly different from those of the respectively sentences in (4). In particular, contrasts such as the following suggest that the adjective respective takes scope strictly within the NP in which it occurs:

(ii) a. Intel and Microsoft combined their respective assets.

b.\#Intel and Microsoft combined their assets respectively.

We thus set aside the adjective respective in the rest of this paper. See Gawron and Kehler (2002) for an analysis of respective that captures its strictly local scope correctly.

${ }^{3}$ Other expressions whose interpretations are similarly sensitive to the order of mention include successively, 
(5) a. John bought the book and Bill bought the CD.

b. John ran and Bill danced.

c. John read the book and Bill listened to the CD.

d. John gave the book to Sue on Wednesday and gave the CD to Mary on Thursday.

The difficulty that the examples in (4) pose essentially lies in the fact that they seem to require having access to the denotations of parts of a phrase. For example, the meaning ran is not retrievable from the boolean conjunction $\lambda x \cdot \operatorname{ran}(x) \wedge \operatorname{danced}(x)$ that is standardly taken to be the meaning of ran and danced. Thus, on the assumption that the boolean conjunction analysis of and is correct, the proper analysis of these sentences would seem to require violating the principle of compositionality at least in its strictest formulation, which dictates that, once the meaning of a phrase is constructed, the grammar should no longer have direct access to the meanings of its parts. Things are especially tricky in examples like (4c) and (4d), where neither the whole coordinate structure nor the conjuncts is even a constituent in the standard sense. So far as we are aware, there is no explicit analysis of these respectively/NCC interactions in the literature. In particular, it is worth noting that the proposals by Gawron and Kehler (2004) and Chaves (2012) that we review below both fail to extend to these NCC cases since they assume phrase structure-based syntax for formulating their analyses (although to be fair, the semantic analysis that Gawron and Kehler (2004) propose does not depend in any crucial way on the syntactic assumptions they make).

One might object to the characterization we have just given (see, e.g., Chaves (2012) and Schwarzschild (1996) - but see Gawron and Kehler (2002) for a critique of Schwarzschild (1996); we address Chaves's (2012) approach in detail in Sect. 4.1.3): at least cases like (4a) can be dealt with by an independently needed mechanism for yielding the so-called cumulative readings of plurals (Scha 1981):

(6) 700 Dutch companies used 10,000 American computers.

In the cumulative reading of (6), a set of 700 Dutch companies is related to a set of 10,000 American computers in the ' $x$-used- $y$ ' relation. The sentence does not specify which particular company used which particular computer, but it only says that the total number of companies involved is 700 and the total number of computers involved is 10,000.

The 'respective' reading in (4a) could then be thought of as a special case of this cumulative reading. Unlike the more general cumulative reading, the 'respective' reading is sensitive to an established order among elements in each of the conjoined or plural terms (that is, (4a) is false in a situation in which John bought the CD and Bill bought the book), but one could maintain that the core compositional mechanism is the same.

However, an attempt to reduce the 'respective' reading to the cumulative reading fails, at least if we adhere to the conventional assumption about the cumulative reading that it is induced by a lexical operator that directly applies to the meanings of verbs. ${ }^{4}$ As should be

progressively and increasingly:

(i) Robin, Terry and Leslie got successively higher grades on the SAT.

\footnotetext{
${ }^{4}$ At the final stage of revising this paper, we became aware of Schmitt (2013), which proposes to generalize
} 
clear from the examples in $(4 \mathrm{~b}-\mathrm{d})$, it is not just co-arguments of a single verb that can enter into the 'respective' relation. Thus, a lexical operator-based approach is not general enough. ${ }^{5}$

But we think there is a grain of truth in this attempt to relate cumulative and 'respective' readings. The 'violation' of compositionality under discussion exhibited by 'respective', symmetrical and summative readings arises only in connection with coordinated or plural expressions. (Examples involving symmetrical and summative predicates are introduced below.) Thus, instead of claiming that these constructions pose serious challenges to the tenet of compositionality (as some authors indeed have; cf. Kay 1989), it seems more reasonable to question the assumption that conjunction in natural language denotes logical, boolean conjunction, and reformulate the semantics of plural and conjoined expressions in such a way that we can capture the relevant readings of these sentences by still adhering to the principles of compositionality fully.

Building on related ideas explored by previous authors, in particular, Gawron and Kehler (2004) and Barker (2007), we argue precisely for such an approach in this paper. In fact, in the case of conjoined NPs, which are standardly taken to denote sums (or, more correctly, joins in a semilattice, as a reviewer reminds us, since sums are constructs in the translation language, not the model - but we'll stick to the informal locution of plurals 'denoting' sums for convenience), the issue of compositionality is already moot since the denotation itself $(\mathbf{j} \oplus \mathbf{b})$ retains the internal structure of the conjunction that can be accessed by other operators such as the distributivity operator commonly assumed in the semantics literature (compare this situation to the generalized conjunction of the lifted versions of the individual terms $\lambda P . P(\mathbf{j}) \wedge$ $P(\mathbf{b})$, for which the individual parts are no longer directly accessible). As shown in detail for 'respective' readings by Gawron and Kehler (2004), by generalizing this approach to nonNP-type meanings, the complex semantics that 'respective' readings and related phenomena exhibit can be uniformly handled by modelling the meanings of expressions involving plurals or conjunction by a structured object - either (generalized) sums (Gawron and Kehler 2004), tuples (Winter 1995; Bekki 2006), or multisets, as we propose in this paper.

The idea, in a nutshell, is to keep track of the meanings of components (e.g. the meanings of each verb ran and danced for the conjoined VP ran and danced) as distinct elements of some complex data structure (which we formally model as multisets) so that they can be separately retrievable from the meaning of the whole conjoined expression. The present paper extends this approach to the other two empirical phenomena (symmetrical and summative predicates), as well as embedding the analysis in a flexible syntax-semantics interface. As will become clear below, our choice of multisets over sums for the underlying data structure departing, in this respect, from Gawron and Kehler (2004) - is primarily motivated by the need to generalize the analysis to the other two phenomena (see Sect. 4 for discussion). We formulate our analysis in a version of Type-Logical Categorial Grammar (TLCG) called Hybrid TLCG (Kubota 2010, 2015; Kubota and Levine 2013a, 2014a). This has the advantage that the interactions with NCC exhibited by data such as (4c) and (4d) (and their counterparts involving symmetrical and summative predicates introduced below) become straightforward. This is especially important since interactions between NCC and each of these phenomena

the mechanism of cumulativity beyond this conventional assumption. Whether this approach generalizes to interactions between 'respective' readings and related phenomena with NCC of the sort discussed in the present paper is an open question.

${ }^{5}$ An event-based analysis (along the lines, e.g., of Lasersohn (1992)) is conceivable for examples like (4b). See Sect. 4.1 for a brief comment on event-based approaches. 
have long been known to pose significant problems for analyses of coordination (see, e.g., Abbott (1976), Jackendoff (1977) and Beavers and Sag (2004) for some discussion), and a fully general solution is still missing in the previous literature.

Having laid out the main goals of the paper, we now turn to the specifics of each of the three phenomena. For the 'respective' reading, note first that if we remove the adverb respectively, the sentences still have the 'respective' reading as one of their possible interpretations.

(7) John and Bill bought the book and the CD.

But in this case, the sentence is multiply ambiguous. For example, in (7), both the subject and object NPs could be construed collectively: the two people bought the two things together. The sentence also allows for readings in which only the subject or the object NP exhibits a distributive reading (e.g. 'John bought the book and the CD and Bill also bought the book and the CD'). The presence of the adverb respectively disambiguates the interpretation to the 'respective' reading.

In the 'respective' readings involving conjunction (rather than plurals) as in the examples in (4), the $n$-th conjunct in one term is matched up with the $n$-th conjunct in the other term. As noted by many (cf., Pullum and Gazdar (1982); Kay (1989); Dalrymple and Kehler (1995); McCawley (1998), among others), if the order of elements is clear from the (non-linguistic) context, not just conjoined NPs but plural NPs can also enter into 'respective' predication, as in the following examples (in particular, Dalrymple and Kehler (1995) contains many naturally occurring examples of this type and discusses several discourse-oriented factors that crucially affect the acceptability of such sentences):

(8) a. The three best students received the three best scores, respectively.

b. A section cut downward from the surface through the various soil horizons forms the soil profile. From the surface downward, the major soil horizons are designated $\mathrm{A}, \mathrm{B}$, and $\mathrm{C}$, respectively.

(Dalrymple and Kehler 1995)

McCawley (1998) also notes that, when there are more than two plural or conjoined terms in the sentence, multiple 'respective' relations can be established among them. Disambiguation with respectively works in the same way as in the simpler examples, with the consequence that (9b) with a single respectively is ambiguity whereas (9a) with two occurrences of respectively is unambiguous:

(9) a. George and Martha sent a bomb and a nasty letter respectively to the president and the governor respectively.

b. George and Martha sent a bomb and a nasty letter to the president and the governor respectively.

As we discuss below, the availability of this multiple 'respective' reading turns out to be particularly important in formulating a compositional semantic analysis of 'respective' readings. Moreover, a parallel multiple dependency is observed with symmetrical predicates, posing a severe problem for the compositional analysis of same and different by Barker (2007, 2012) (see Sect. 4.1.2). So far as we are aware, Gawron and Kehler (2004) was the first to propose a formally explicit solution for this problem in the analysis of 'respective' readings, and our own analysis generalizes it to all of the three phenomena we treat in this paper. 
Turning now to symmetrical predicates, note first that symmetrical predicates such as same, different, similar and identical exhibit an ambiguity between the so-called 'internal' and 'external' readings (Carlson 1987).

(10) a. The same waiter served Robin and poured the wine for Leslie.

b. Different waiters served Robin and poured the wine for Leslie.

When uttered in a context in which some waiter is already salient (for example, when (10a) is preceded by I had a very entertaining waiter when I went to that restaurant last week, and yesterday evening...), the same waiter in (10a) anaphorically refers to that individual already introduced in the discourse. This is called the EXTERNAL READING. But this sentence can be uttered in an 'out of the blue' context too, and in this case, it simply asserts that the individual who acted as Robin's server and the one who poured Leslie's wine were identical, and that that individual was a waiter-the so-called INTERNAL READING. The external reading reflects just an anaphoric use of these expressions and does not pose a particularly challenging problem for compositional semantics. For this reason, we set it aside and focus on the internal reading in the rest of the paper (but see Sect. 3.2.2, where we briefly discuss a possibility in which the internal and external readings may be related in our setup).

The distribution of the internal reading of symmetrical predicates is remarkably similar to that of 'respective' readings. First, like 'respective' readings, the internal reading is available in all types of coordination:

a. John and Bill read \{the same book/different books\}. (NP coordination)

b. $\{$ The same waiter/Different waiters $\}$ served Robin and poured the wine for Leslie.

(VP coordination)

c. John read, and Bill reviewed, \{the same book/different books\}.

d. I gave $\{$ the same book/different books $\}$ to John on Wednesday and to Bill on Thursday.

(DCC)

Examples like (11c) and (11d) are especially problematic since they show that deletion-based analyses of NCC (which derive, for example, the RNR example (11c) from an underlying source John read the same book and Bill reviewed the same book) do not work (Abbott 1976; Jackendoff 1977; Carlson 1987; Kubota 2015; Kubota and Levine 2015).

Second, both plural and conjoined expressions induce the internal reading. Thus, by replacing John and Bill in (11a) by the men, both the external and internal readings are available:

(12) The men read $\{$ the same book/different books .

Third, just like multiple 'respective' readings, multiple internal readings are possible:

a. John and Bill bought the same book at the same store.

b. John and Bill bought the same book at different stores.

c. John and Bill bought the same book at the same store on the same day for the same price.

Note moreover that the 'respective' reading and the internal reading interact with one another: 
(14) a. John and Mary showed the same book to his brother and her sister, respectively.

b. The White House proposed, and the Justice Department formally recommended, different codes of conduct to the Boy Scouts and the CIA Operations Section respectively on the same day.

These similarities, especially the fact that the two phenomena interact with one another systematically as in (14), strongly suggest that one and the same mechanism is at the core of the compositional semantics of these constructions.

The parallel distributional pattern in fact extends to the interpretations of summative predicates such as a total of $N$ and $N$ in total as well. The problem that summative predicates pose for the syntax-semantics interface is best known in the context of RNR, in examples such as the following (Abbott 1976):

(15) John spent, and Bill lost, a total of $\$ 10,000$ last year.

Just like the internal reading for symmetrical predicates (cf. (11c)), (15) has a reading that is not equivalent to its 'paraphrase' with clausal coordination:

(16) John spent a total of $\$ 10,000$ last year and Bill lost a total of $\$ 10,000$ last year.

But the summative reading exhibited by (15) (where $\$ 10,000$ corresponds to the sum of amounts that respectively satisfy the two predications) is not limited to RNR. The same reading is found in the full range of coordination constructions:
a. The two men spent a total of $\$ 10,000$.
(NP coordination)
b. A total of $\$ 10,000$ was spent and lost.
(VP coordination)
c. John donated a total of $\$ 10,000$ to Red Cross on Thursday and to Salvation Army on Friday.
(DCC)

Note also that here too, both plural NPs (as in (17a)) and conjoined expressions (as in $(17 \mathrm{~b}, \mathrm{c}))$ can enter into summative predication.

Moreover, just as with 'respective' readings and internal readings, iterated summative readings are also possible, and these phenomena interact with one another:

a. A total of three boys bought a total of ten books.

b. John collected, and Mary got pledges for, a total of $\$ 10,000$ for charity from his family and her clients, respectively.

c. John gave, and Bill lent, a total of $\$ 10,000$ to $\left\{\begin{array}{l}\text { the same student } \\ \text { different students }\end{array}\right\}$.

We are not aware of any explicit previous analysis that accounts for the interactions of these phenomena with each other exemplified by $(18 \mathrm{~b}, \mathrm{c})$ and $(14)$ above. We take it that these examples provide a particularly strong argument for a unified analysis of these phenomena.

In the next section, we propose a unified analysis of 'respective', symmetrical and summative predicates that accounts for the parallels and interactions among these phenomena straightforwardly. The key idea that we exploit is that conjunction and plural expressions (as well as symmetrical and summative predicates) denote multisets, that is, sets that allow 
for duplicate occurrences of identical elements, and that the same 'respective' predication operator mediates the complex (yet systematic) interactions they exhibit that pose apparent challenges to compositionality. While the semantics of each of these phenomena have been studied extensively in the previous literature by several authors, to our knowledge, a unified and fully detailed compositional analysis - especially one that extends straightforwardly to cases involving interactions with NCC - has not yet been achieved. (But see Chaves (2012) for an insightful recent attempt, some of whose key ideas we inherit in our own analysis; see Sect. 4.1.3 for a comparison.) We believe that the unified analysis we offer below clarifies the compositional mechanism underlying these phenomena, in particular the way it interacts with the general syntax and semantics of coordination including both standard constituent coordination and NCC.

\subsection{Some residual issues}

Before moving on to the analysis, we would like to address several residual issues, some of which might initially appear to threaten our unified treatment of 'respective', symmetrical and summative predicates presented in the next section.

\subsubsection{Apparent non-parallels between 'respective', symmetrical and summative predicates}

As we have discussed above, the analysis presented below builds on the idea that a single common mechanism is at the core of the semantics of the three phenomena reviewed above. One might, however, note that these phenomena do not seem to behave in a completely parallel fashion. While we admit that we do not currently have a complete account of such non-parallels (suggesting, however, some possibilities to explore in what follows), jumping to the conclusion that these non-parallels threaten a unified analysis is too hasty. We believe that in each such case, the superficial difference should ultimately be attributed to independent factors orthogonal to the core semantic mechanism common to the three phenomena.

Interactions with universal quantifiers The first alleged discrepancy among the three phenomena comes from examples involving universal quantifiers every and each. Note first that every and each license internal readings of symmetrical predicates quite readily:

a. Every student read $\{$ the same book/a different book\}.

b. Each student read $\{$ the same book/a different book\}.

Given the parallel between symmetrical predicates on the one hand and 'respective' and summative predicates on the other noted above, one might then expect the latter two to induce the relevant readings with universal quantifiers similarly. This expectation seems initially falsified by data such as the following:

a. $\{$ Each/Every $\}$ student read a total of 20 books.

b.\#\{Each/Every $\}$ student read War and Peace, Anna Karenina, and The Idiot, respectively. 
These sentences lack the relevant 'respective'/summative readings. (20a) can be interpreted only on the strictly distributive reading of each/every (where each of the students read a total of 20 books separately) and (20b) is simply infelicitous since the adverb respectively is incompatible with the distributive reading of each and every.

However, it has been noted in the literature that the relevant readings are available at least in certain examples:

a. Three copy-editors (between them) caught every mistake in the manuscript. (Kratzer 2007)

b. (...) it is essential that an agreement be reached as to the costs that each party will respectively bear.

(Chaves 2012)

There seem to be several factors involved in the contrast between the examples in (21) and those in (20), one of which is arguably pragmatic. For example, in the case of $(21 \mathrm{~b})$, an implicit dependency between the members of the two sets in question - which in this case is supported by the fact that one is an implicit argument of the other (each party is a bearer of the costs) - seems to facilitate the relevant reading. Another relevant factor seems to be realtime sentence processing. Note that, in both of the examples in (21), the plural expression precedes the NP containing each/every. ${ }^{6}$ This seems to facilitate the 'respective'/summative predication somehow. ${ }^{7}$ In general, linear order (or 'evaluation order' in the sense of Barker and Shan (2015)) often seems to crucially affect the possible range of interpretations of scopal expressions. See, for example, Barker and Shan (2015) for an explicit grammar fragment which takes this factor into account for the ordinary scope interpretations of quantifiers. We believe that the acceptability contrast in (20) vs. (21) should ultimately receive explanations along similar lines. Thus, questions remain, but the main point here is that the NP type (plural vs. universal quantifier) cannot be the sole factor determining the availability of 'respective', symmetrical and summative interpretations, given the existence of examples such as $(21)$.

The incompatibility of overt respectively with symmetrical and summative predicates Unlike 'respective' readings, symmetrical and summative predicates are incompatible with the adverb respectively:

(22) a:\#John and Bill read \{the same book/different books\}, respectively.

b.\#John and Bill spent a total of $\$ 10,000$, respectively.

\footnotetext{
${ }^{6}$ Kratzer (2007) attributes the contrast between (21a) and Every copy editor caught 500 mistakes in the manuscript (which does not induce the relevant summative reading) to differences in grammatical relations. However, see Champollion (2010) for a discussion that grammatical relation is not the relevant factor.

${ }^{7}$ But note that linear order cannot be the sole determining factor. The following example induces the 'respective' reading with a universal quantifier, despite the fact that the quantifier linearly precedes the plural (it is also anomalous in involving disjunction rather than conjunction; for disjunction's (apparently surprising) ability to license 'respective' readings, see Gawron and Kehler (2004)):
}

(i) For every document, she had to translate it to Russian or Bielo-Russian respectively. (Chaves 2012)

Here, the PP containing the quantifier is fronted, which arguably affects processing, and so it isn't too surprising that this example differs from simpler examples like those in (20) in its possible interpretation. That said, the exact licensing condition for 'respective' readings for quantifiers is still quite elusive. 
As noted by Chaves (2012) (among others), the function of the adverb respectively is to ensure that the bijection established is in accordance with the contextually provided ordering (where the relevant ordering is either given by the linguistic context (i.e. order of mention) or the pragmatic context). Although the technical analysis we propose below makes use of the same mechanism for establishing a bijective mapping in the three phenomena, the nature of the ordering is crucially different in the case of respectively sentences on the one hand and symmetrical and summative predicates on the other. In the latter two cases, the relevant ordering is introduced purely for the sake of ensuring that a proper bijective mapping is established. Thus, since there is no contextually salient ordering involved in examples like (22), the use of respectively is infelicitous. ${ }^{8}$

\subsubsection{Non-coordinate RNR and dependent clusters}

It is well-known that RNR is not restricted to coordination (Hudson 1976; Phillips 1996):

a. Stone also suggests that Nixon knew of, though he did not attempt to participate in, US attempts to assassinate Fidel Castro.

(Boston Sunday Globe movie section; example provided by an anonymous reviewer)

b. The people who liked, easily outnumbered the people who disliked, the movie we saw yesterday.

Interestingly, as an anonymous reviewer points out, some of these 'non-coordinate' RNR can induce the 'respective' reading and the internal reading of symmetrical predicates: ${ }^{9}$

a. John defeated, \{whereas/although\} Mary lost to, the exact same opponent.

b. John defeated, whereas Mary lost to, Sam and Kim, respectively.

The reviewer takes frontability of whereas and although-clauses to be a test for non-coordinatehood, and remarks that the availability of the relevant readings in (24) would be puzzling for an

\footnotetext{
${ }^{8} \mathrm{~A}$ reviewer notes that this leaves open a possibility that the ordering that a symmetrical predicate refers to happens to be identical to the contextually established one. This would be difficult with same, which involves only a single fixed entity (for which ordering is irrelevant), but seems indeed possible with different in the right kind of context, such as the following one provided by the reviewer. Suppose John and Bill travelled to Africa and East Asia, respectively, in which two particular types of hepatitises (types A and B, respectively) are widespread. In this context, the following sentence, with the definite article the crucially invoking reference to the contextually understood set of hepatitises (together with the contextually understood ordering/linking), seems quite unexceptional:
}

(i) John and Bill were diagnosed with the two different types of hepatitis, respectively.

We want to acknowledge here however that, as it stands, the formal analysis we present below does not take into account the possible role of the definite article the in examples like (i). We believe that our analysis is not incompatible with a refinement that takes into account such further subtleties. This is left for future work.

${ }^{9}$ The reviewer also notes that the following example however does not induce the summative reading:

(i) John spent, whereas Mary lost, a total of $\$ 10,000$.

This seems to be due to the fact that the 'contrast' discourse relation lexically invoked by whereas is inherently incompatible with the pragmatics of summative interpretation (in which the two clauses need to be construed to pertain to some common point, rather than being in contrast with one another). 
analysis (such as the one we present below) that holds that these readings are tied to coordination.

But it should be noted that syntactic coordinatehood is not the relevant factor here. As pointed out by Beavers and Sag (2004), the disjunction or is seriously degraded in the internal reading, and other types of non-coordinate RNR whose (truth-conditional) meanings do not correspond to conjunction similarly fail to induce the relevant readings:

a.\#John hummed, or Mary sang, the same tune.

(Beavers and Sag 2004)

b.\#The people who liked, easily outnumbered the people who disliked, the same movie.

We think that the relevant generalization is whether the construction in question has the meaning of conjunction. ${ }^{10}$ Whereas and although are truth-conditionally equivalent to conjunction, with an extra pragmatic function of indicating a particular discourse relation (some kind of contrast) between the two clauses. Since the analysis we present below is predicated of the conjunctive meaning of and rather than its syntactic coordinatehood, the examples in (24), rather than undermining our analysis, in fact provide further corroboration for it.

More challenging to our approach are cases like the following, in which dependent cluster formation interacts with comparatives. As noted by Moltmann (1992) and Hendriks (1995), comparatives license the internal reading of same (but this does not seem to be possible with other symmetrical predicates such as different and similar), and this works in examples involving nonconstituents in the comparative clause also:

a. I gave the same girl more books on Saturday than CDs on Sunday.

b??I gave $\{$ different/similar\} girls more books on Saturday than CDs on Sunday.

We do not know of any compositional analysis of internal readings of symmetrical predicates that can account for the interaction of same and comparatives in examples like (26a). ${ }^{11}$ It is tempting to speculate that the internal reading here is supported by some core meaning (something like 'parallel predications' involving multiple clauses) common to conjunction and comparatives, and that, once this core meaning is identified, then the existing analyses of internal readings would straightforwardly carry over to cases like (26a). However, given the lack of a concrete proposal, we simply leave this as an open issue for future study.

${ }^{10} \mathrm{~A}$ reviewer notes that the following examples are acceptable:

(i) a. The people who initially opPosed, ended up SUPPORTING, the very same proposal.

b. You're floundering if you say you oppose, then later SUPPORT, the same proposal.

The native speakers we consulted uniformly rejected (ia) on the internal reading for same. For (ib), the judgment was not so clear; most of our informants reported that it was a borderline example. This latter fact makes sense given that the if $A$ then $B$ part in (ib) is not a conditional statement, but rather the then clause is a temporal adjunct clause inside the antecedent of a conditional. Thus, the $A$ then $B$ is a hidden conjunction with an additional temporal precedence information imposed on it (which presumably is a confounding factor in inducing the relevant internal reading).

${ }^{11}$ Note that, though Heim (1985) takes a parallel between comparatives and same and different as a starting point for her analysis of the latter two, she does not provide any explicit analysis of examples like (26a) in which the two phenomena interact with one another (nor is it obvious how her account could be extended to such examples). 


\subsubsection{Non-local 'respective' predication and island constraints}

All of the three phenomena involve interdependency between two (or more) terms in the interpretation. This interdependency has sometimes been analyzed in terms of a non-local movement (or movement-like) operation (as in Barker's (2007) analysis of same via 'parasitic scope'; cf. Sect. 4.1.2) and sometimes via a chain of local operations (as in Gawron and Kehler's (2004) analysis of 'respective' readings; cf. Sect. 4.1.1). One might then wonder whether any empirical argument can be made for one or the other type of analysis.

This is an interesting and important question, but unfortunately, it is currently unclear to us whether any decisive case can be made for either approach (see also the discussion in Sect. 5 below; purely from a formal perspective, the 'local' approach to 'respective' readings $a$ la Gawron and Kehler and the 'non-local' approach a la Barker can be shown to be equivalent under certain assumptions). The main challenge that this task faces is that the empirical question of what exactly is the nature of the relevant locality constraints (if there are any) for 'respective' and symmetrical predicates is heavily underinvestigated, in contrast to other empirical domains such as long-distance dependencies. In fact, so far as we were able to identify, this issue has been explicitly discussed only with respect to symmetrical predicates, and in a fairly cursory manner in the literature. The major disputes were all from the late 80s and early 90s, and since then there does not seem to have been any extensive or systematic investigation: Carlson (1987) claimed that the internal readings of same/different obey the same syntactic island constraints as filler-gap dependencies, but this observation was disputed by Dowty (1985) and Moltmann (1992) (see also Heim (1985) for some discussion). We concur with these latter authors. Note in particular the following cases, all of which seem to induce the internal reading for the symmetrical predicates relatively easily: ${ }^{12}$

a. Robin and Leslie believe that the acquisition of $\left\{\begin{array}{c}\text { the same } \\ \text { different }\end{array}\right\}$ skill sets is crucial to success in the business world. (Subject island violation on the de re reading)

b. [Robin and Leslie usually agree about who's a bad singer at a karaoke party, and they both get immediately mad when such a person starts singing.]

But today, something weird happened: Robin and Leslie got mad when DIFFERENT people started singing.

(Adjunct island violation)

c. The Smiths ${ }_{1}$ and the Jones's ${ }_{2}$ go to the same psychiatrist and to different $_{2}$ psychiatrists respectively. (Coordinate Structure Constraint violation; Dowty $(1985,6))$

Similar facts seem to hold for the 'respective' reading:

\footnotetext{
${ }^{12}$ Though the nature of 'covert movement' is still considerably unclear and controversial (see, for example, Ruys and Winter (2010) for a recent review), this at least means that a movement-based analysis of these expressions (via QR) cannot be motivated in terms of island-sensitivity. On the other hand, if one takes island effects to be by-products of functional factors such as constraints on real-time processing and felicity conditions on discourse (Deane 1991; Kluender 1992, 1998; Kehler 2002; Hofmeister and Sag 2010), what the above data suggests is that the processing and discourse factors which come into play in filler-gap dependencies are not the same ones which govern the interpretation of 'respective' and symmetrical predicates. In the former case, island violation does result in reduced acceptability, but it can be ameliorated by manipulating various nonstructural factors; in the latter case, island violation simply does not seem to arise to begin with, as evidenced by the near-perfect acceptability of the examples in (27) and (28).
} 
(28) a. Robin and Leslie thought that studying category theory and intuitionistic logic respectively would be all that was needed for success. (Subject island violation)

b. Robin and Leslie got home before the train and the bus stopped running respectively.

(Adjunct island violation)

c. Robin and Leslie named someone who was innocent and guilty respectively.

(Complex NP constraint violation)

Our own analysis, presented in the next section, is formulated in terms of a movementlike operation, but we do not ourselves commit to the idea that this entails that 'respective' readings, etc., are sensitive to islands, since we do not take island constraints to reflect combinatoric properties of the grammar. In any event, a more thorough empirical investigation is needed to settle the issue in one way or another, especially from an experimental point of view.

\section{The compositional semantics of 'respective' predication}

In this section, we present a unified analysis of 'respective', symmetrical and summative predicates in a variant of categorial grammar (CG) called Hybrid Type-Logical Categorial Grammar (Hybrid TLCG; Kubota 2010, 2014, 2015; Kubota and Levine 2013a, 2014a). Key to our proposal is the idea that the same underlying mechanism of pairwise predication is involved in the semantics of these phenomena. This analytic idea itself is theory-neutral, but we show that formulating the analysis in Hybrid TLCG enables us to capture the complex yet systematic properties of these phenomena that are relevant for the general architecture of the syntax-semantics interface of natural language particularly transparently. More specifically, the order-insensitive mode of implication involving the 'vertical slash' $(\uparrow)$, the key novel feature of Hybrid TLCG (explained below), enables a unified analysis of the two essential properties of these phenomena identified in Sect. 2: (i) interactions with NCC and (ii) multiple dependency that these predicates exhibit including the interactions of the three phenomena with one another.

\subsection{The syntax-semantics interface of Hybrid TLCG}

In this section, we introduce the framework of Hybrid TLCG. We start with a gentle introduction to the Lambek calculus, illustrating its linguistic application with the analyses of RNR and DCC, and then extend the fragment to Hybrid TLCG. The key point of this extension is that, by adding just one new mechanism to the Lambek calculus, the notion of 'movement' from derivational approaches can be straightforwardly modelled in CG. From the CG perspective, the new ingredient is really just another kind of implication (or slash) - called the VERTICAL SLASH $(\uparrow)$ - which, unlike the forward and backward slashes in the Lambek calculus, does not encode linear order in the syntactic category. After introducing the relevant rules, we illustrate how this new mechanism enables a straightforward analysis of quantifier scope (covert movement) and wh-movement (overt movement). Due to space limitations, the presentation of Hybrid TLCG here is somewhat compact. The reader is referred to Kubota (2015), and especially Kubota and Levine (2014b), for a more leisurely exposition. Readers who are interested in the formal properties of Hybrid TLCG should consult Kubota and Levine (2014c) and Moot (2014). The latter work is especially important in that it proves 
some important properties of Hybrid TLCG, such as those pertaining to decidability (see also Sect. 4.3 below for some discussion on empty operators and decidability).

\subsubsection{The Lambek calculus in labelled deduction}

Following Morrill (1994), we start by recasting the Lambek calculus in the 'labelled deduction' format (see also Oehrle (1994)), writing linguistic expressions as tuples $\langle\phi, \sigma, \kappa\rangle$ of phonological form $\phi$, semantic translation $\sigma$, and syntactic type $\kappa$ (or category-following the convention in TLCG, we use the terms 'syntactic type' and 'syntactic category' interchangeably), as in the following sample lexicon:
a. john; $\mathbf{j} ; \mathrm{NP}$
c. walks; walk; $\mathrm{NP} \backslash \mathrm{S}$
b. mary; m; NP
d. loves; love; $(\mathrm{NP} \backslash \mathrm{S}) / \mathrm{NP}$

Complex categories are built from atomic categories (including S, NP and N) recursively with the connectives forward slash / and backward slash \(to which the vertical slash \will be later added). We adopt the Lambek-style notation of slashes, where what appears under the slash $(A$ in $A \backslash B)$ is always the argument. Parentheses for a sequence of the same type of slash is omitted, as in $\mathrm{S} / \mathrm{NP} / \mathrm{NP}, \mathrm{NP} \backslash \mathrm{NP} \backslash \mathrm{S}$ and $\mathrm{S}\lceil\mathrm{NP} \backslash \mathrm{NP}$, which are abbreviations of $(\mathrm{S} / \mathrm{NP}) / \mathrm{NP}, \mathrm{NP} \backslash(\mathrm{NP} \backslash \mathrm{S})$ and $(\mathrm{S} \backslash \mathrm{NP}) \uparrow \mathrm{NP}$, respectively.

The following proof in (30) illustrates how larger linguistic expressions are built from smaller ones using the rules of grammar, of which we first introduce the Elimination rules for / and \in (31) (these rules should be thought of as directional variants of modus ponens $B \rightarrow A, B \vdash A)$. Here, a transitive verb, of category $(\mathrm{NP} \backslash \mathrm{S}) / \mathrm{NP}$, is combined with its two arguments on the right (object) and left (subject).

a. Forward Slash Elimination

$$
\frac{a ; \mathcal{F} ; A / B \quad b ; \mathcal{G} ; B}{a \circ b ; \mathcal{F}(\mathcal{G}) ; A} / \mathrm{E}
$$

b. BackWard Slash Elimination

$$
\frac{b ; \mathcal{G} ; B \quad a ; \mathcal{F} ; B \backslash A}{b \circ a ; \mathcal{F}(\mathcal{G}) ; A} \backslash \mathrm{E}
$$

The Elimination rules can roughly be thought of as subcategorization cancellation rules. Note that, by applying the rules in (31), the right surface word order is obtained in (30), paired with the right meaning. The prosodic effect of these rules is string concatenation. / $(\backslash)$ combines the argument to the right (left) of the functor. The semantic effect is function application in both cases.

TLCG takes the analogy between language and logic quite literally. Thus, in addition to the Elimination rules there are also Introduction rules for the two connectives / and $\backslash$. These rules should be thought of as directional variants of implication introduction (or hypothetical reasoning; drawing the conclusion $A \rightarrow B$ given a proof of $B$ by hypothetically assuming $A$ ). 
a. Forward Slash Introduction

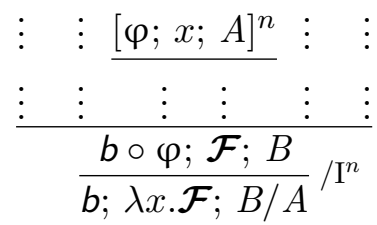

b. Backward Slash Introduction

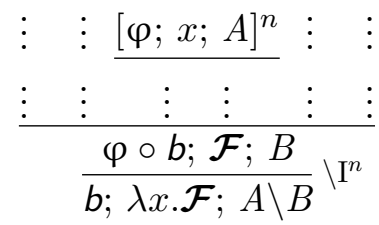

We first illustrate the workings of these rules with an analysis of RNR and DCC, and then come back to the relevant formal details. The significance of the /I and $\backslash \mathrm{I}$ rules is that they permit the reanalysis of any substring of a sentence as a (derived) constituent, which gives (TL)CG a distinct advantage over other syntactic theories in analyzing phenomena such as coordination. This is illustrated in (34), which analyzes the string John loves in (33) as a full-fledged constituent of type S/NP.

John loves, but Bill hates, Mary.

$$
\text { (1) } \rightarrow \frac{\text { john } ; \mathbf{j} ; \mathrm{NP} \frac{[\varphi ; x ; \mathrm{NP}]^{1} \quad \text { loves; love } ;(\mathrm{NP} \backslash \mathrm{S}) / \mathrm{NP}}{\text { loves } \circ \varphi ; \text { love }(x) ; \mathrm{NP} \backslash \mathrm{S}}}{(2) \rightarrow \frac{\text { john } \circ \text { loves } \circ \varphi ; \text { love }(x)(\mathbf{j}) ; \mathrm{S}}{\text { john } \circ \text { loves } ; \lambda x . \operatorname{love}(x)(\mathbf{j}) ; \mathrm{S} / \mathrm{NP}} / \mathrm{I}^{1}} / \mathrm{E}
$$

By hypothesizing a direct object NP, we first prove an S (1)). (The brackets around premises identify hypotheses, and the index is for keeping track of where the hypothesis is withdrawn in the proof.) At this point, since the phonology of the hypothesized NP $\varphi$ appears on the right periphery, we can apply /I (2) to withdraw the hypothesis. Intuitively, what is going on here can be paraphrased as follows: since we've proven that there is a complete $\mathrm{S}$ by assuming that there is an NP on the right periphery (1)), we know that, without this hypothetical NP, what we have is something that becomes an S if there is an NP to its right (2)). Note that the lambda abstraction on the corresponding variable in semantics assigns the right meaning (of type $e \rightarrow t$ ) to the derived $\mathrm{S} / \mathrm{NP}$.

In the CG analysis of RNR (see, e.g., Morrill (1994); the original analytic insight goes back to Steedman (1985)) such nonconstituents are directly coordinated as constituents and then combined with the RNR'ed expression as in (35) (we use calligraphic letters $(\mathcal{U}, \mathcal{V}, \mathcal{W}, \ldots)$ for polymorphic variables; copperplate letters $(\mathscr{P}, \mathscr{Q}, \ldots)$ are reserved for higher-order variables (with fixed types)):

$$
\begin{aligned}
& \begin{array}{lr}
\text { and; } & \vdots \\
\lambda \mathcal{W} \lambda \mathcal{V}, \mathcal{V} \sqcap \mathcal{W} ; & \text { bill } \circ \text { hates; }
\end{array}
\end{aligned}
$$

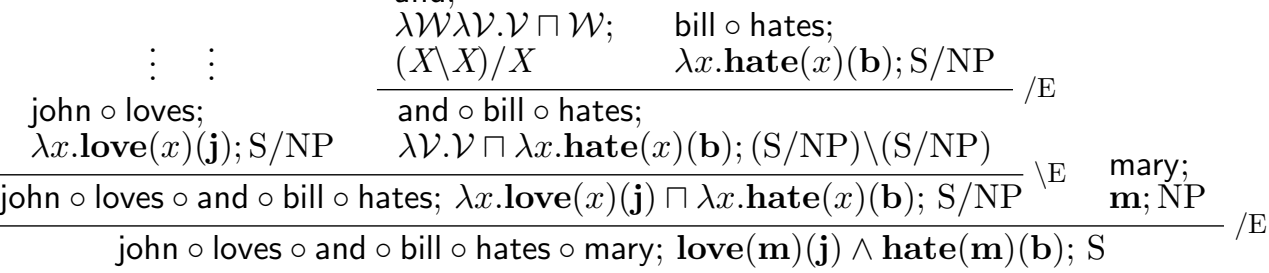

Note in particular that this analysis assigns the right meaning to the whole sentence compositionally (we assume here, for the sake of exposition, that the meaning of and is boolean generalized conjunction (Partee and Rooth 1983); this assumption will be revised in Sect. 3.2 
below). This analysis (and the parallel analysis of DCC discussed below) receives independent motivation from the scopal properties of several semantic operators including generalized quantifiers (Kubota and Levine 2015) and focus-sensitive particles (for the relevant data involving focus-sensitive particles, see Mouret (2006)). The present paper demonstrates that the 'respective' readings, symmetrical predicate, and summative predicates constitute yet another class of phenomena whose semantics argues for this analysis of NCC.

This analysis of RNR immediately extends to DCC. (36) shows the 'reanalysis' of the string Bill the book as a derived constituent. This involves hypothesizing a ditransitive verb and withdrawing that hypothesis after a whole VP is formed:

$$
\frac{[\varphi ; f ; \mathrm{VP} / \mathrm{NP} / \mathrm{NP}]^{1} \text { bill; } \mathbf{b} ; \mathrm{NP}}{\frac{\varphi \circ \text { bill; } f(\mathbf{b}) ; \mathrm{VP} / \mathrm{NP}}{\varphi} / \mathrm{E} \text { the o book; the-bk; NP }} / \mathrm{E}
$$

Then, after like-category coordination, the missing verb and the subject NP are supplied to yield a complete sentence (the last step is omitted):

\begin{tabular}{|c|c|c|c|}
\hline & & $\begin{array}{l}\text { and; } \\
\lambda \mathcal{W} \lambda \mathcal{V} \cdot \mathcal{V} \sqcap \mathcal{W} \\
(X \backslash X) / X\end{array}$ & $\begin{array}{l}\text { john o the o record; } \\
\lambda f . f(\mathbf{j})(\text { the-rc }) \\
(\mathrm{VP} / \mathrm{NP} / \mathrm{NP}) \backslash \mathrm{VP}\end{array}$ \\
\hline & $\begin{array}{l}\text { bill o the o book; } \\
\lambda f . f(\mathbf{b})(\text { the-bk }) \\
(\mathrm{VP} / \mathrm{NP} / \mathrm{NP}) \backslash \mathrm{VP}\end{array}$ & \multicolumn{2}{|c|}{$\begin{array}{l}\text { and } \circ \text { john } \circ \text { the o record; } \\
\lambda \mathcal{V} . \mathcal{V} \sqcap \lambda f . f(\mathbf{j})(\text { the-rc }) ; \\
((\mathrm{VP} / \mathrm{NP} / \mathrm{NP}) \backslash \mathrm{VP}) \backslash((\mathrm{VP} / \mathrm{NP} / \mathrm{NP}) \backslash \mathrm{VP})\end{array}$} \\
\hline /NP/NP & \multicolumn{3}{|c|}{$\begin{array}{l}\text { bill } \circ \text { the } \circ \text { book } \circ \text { and } \circ \text { john } \circ \text { the } \circ \text { record; } \\
\lambda f . f(\mathbf{b})(\text { the-bk }) \sqcap \lambda f . f(\mathbf{j})(\text { the-rc }) ;(\mathrm{VP} / \mathrm{NP} / \mathrm{NP}) \backslash \mathrm{VP}\end{array}$} \\
\hline
\end{tabular}

We now return to some formal details. Since $\circ$ is string concatenation, the fragment up to this point is equivalent to the (associative) Lambek calculus $\mathbf{L}$ (Lambek 1958). This means that a hypothesis can be withdrawn as long as its phonology appears either on the left or right periphery of the phonological representation of the input expression (for a more elaborate setup incorporating the notion of 'multi-modality', see, e.g., Moortgat and Oehrle 1994; Dowty 1996; Muskens 2007; Kubota 2014). Note also that in this formulation, the phonological term labelling, rather than the left-to-right order of the premises in the proof tree (as is more commonly done in the literature of mathematical linguistics), is relevant for the applicability conditions of the /I and $\backslash$ I rules (so far as we are aware, Morrill (1994) was the first to recast the Lambek calculus in this format). This point should be clear from the proof in (34), where we have deliberately placed the hypothetical object NP to the left of the verb in the proof tree. This also means that the order of the two premises in the Elimination rules does not play any role. In practice, we often write premises in an order reflecting the actual word order, for the sake of readability of derivations.

\subsubsection{Extending the Lambek calculus with the 'vertical slash'}

Though the Lambek calculus is notable for its smooth handling of phenomena exhibiting flexibility of surface constituency (such as nonconstituent coordination just illustrated), it 
runs into problems in dealing with phenomena that exhibit 'overt' and 'covert' movement. To model these phenomena, following Oehrle (1994), we introduce a new, order-insensitive mode of implication $\uparrow$ called VERTICAL SLASH, the Introduction and Elimination rules for which are formulated as follows:

a. Vertical Slash Introduction

$$
\begin{array}{ccccc}
\vdots & \vdots & {[\varphi ; x ; A]^{n}} & \vdots & \vdots \\
\vdots & \vdots & \vdots & \vdots & \vdots \\
\frac{b ; \mathcal{F} ; B}{\lambda \varphi . b ; \lambda x . \mathcal{F} ; B\lceil A} & \left\lceil I^{n}\right.
\end{array}
$$

b. Vertical Slash Elimination

$$
\frac{a ; \mathcal{F} ; A \uparrow B \quad b ; \mathcal{G} ; B}{a(b) ; \mathcal{F}(\mathcal{G}) ; A}\lceil\mathrm{E}
$$

Unlike the /I and $\backslash I$ rules, in the $\backslash I$ rule, the missing position of $A$ within $B\lceil A$ is explicitly kept track of via $\lambda$-binding in phonology (note that, as with /, we write the argument to the right; the harpoon is there as a visual aide indicating that the right category $A$ is the argument). This means that we admit functional expressions in the phonological component. Such functional phonologies are applied to their arguments via the $\ E$ rule, whose phonological effect is function application. Note the close parallel between the semantic and phonological operations in these rules. Like / and $\backslash, \uparrow$ is a connective for LINEAR implication. This means that it can bind only one occurrence of a hypothesis at a time. This has an important empirical consequence, as we note below.

As was first noted by Oehrle (1994), hypothetical reasoning with $\lceil$ enables a formal modelling of Montague's (1973) quantifying-in, or what (roughly) corresponds to covert movement in derivational frameworks. This is illustrated in (39) for the $\forall>\exists$ reading for the sentence Someone talked to everyone yesterday:

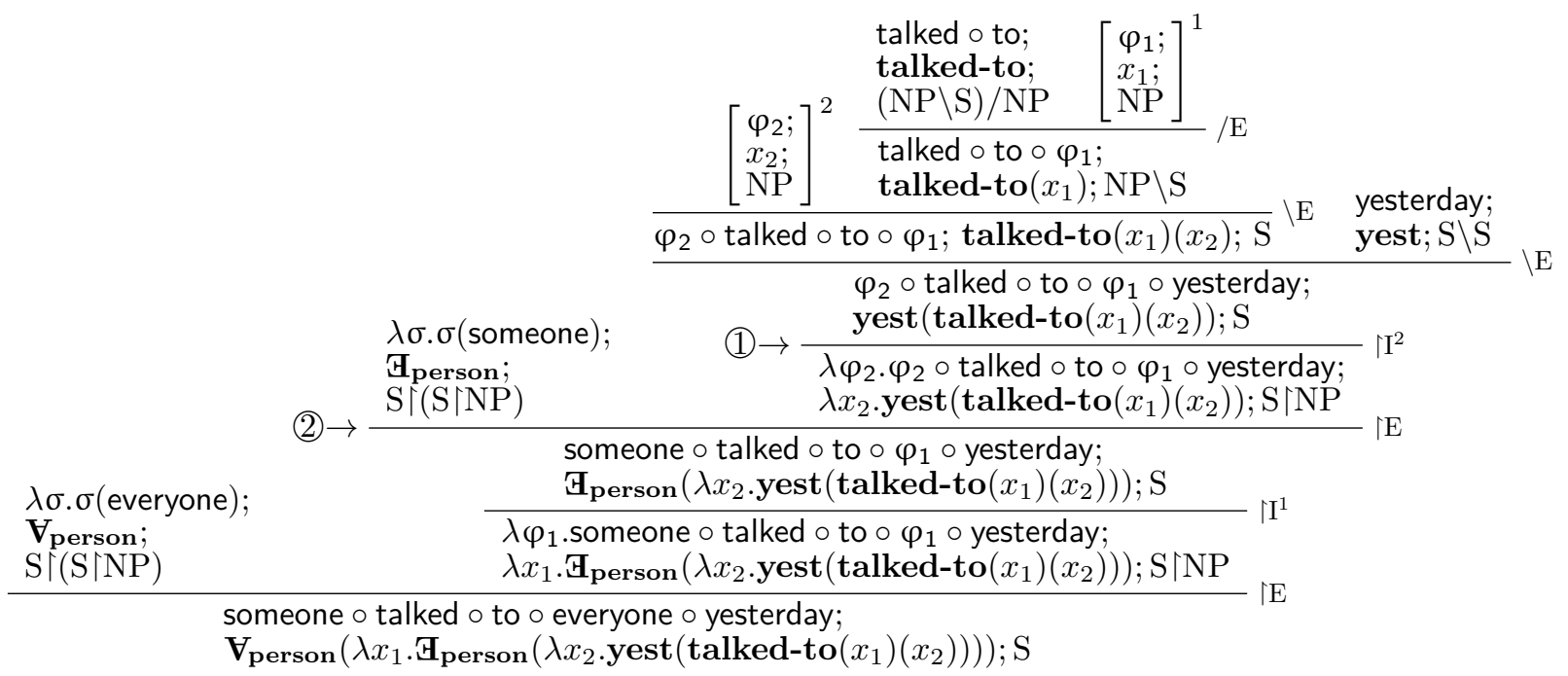

Here, G Gerson abbreviates the term $\lambda P . \exists x[\operatorname{person}(x) \wedge P(x)]$ (similarly for the universal quantifier). Thus, a quantifier has the ordinary GQ meaning, but its phonology is a function of type $(\mathbf{s t} \rightarrow \mathbf{s t}) \rightarrow \mathbf{s t}$ (with st the type of strings). In (39), by abstracting over the position in which the quantifier 'lowers into' in an S, we first form an expression of type S「NP (1), 
a sentence missing an NP inside (phonologically of type st $\rightarrow \mathbf{s t}$ ) where the position of the missing NP is explicitly kept track of via $\lambda$-binding in the prosodic component. By giving this expression as an argument to the quantifier (2), the subject quantifier someone semantically scopes over the sentence and lowers its string phonology to the 'gap' position. The scopal relation between multiple quantifiers depends on the order of application of this hypothetical reasoning. We get the inverse scope reading $(\forall>\exists)$ in this derivation since the subject quantifier is combined with the sentence first. ${ }^{13}$

In the present approach, the difference between overt movement and covert movement comes down to a lexical difference in the 'prosodic action' of the operator that triggers the 'movement' operation. As shown above, covert movement is modelled by an operator which embeds some (non-empty) string in the gap position. Overt movement, by contrast, is modelled by an operator which embeds the empty string in the gap position. Thus, as shown by Muskens (2003), extraction can be analyzed quite elegantly in this type of approach with hypothetical reasoning for $\uparrow$, as in the derivation in (41) for the topicalization example (40).

$$
\text { Bagels }_{i} \text {, Kim gave } t_{i} \text { to Chris. }
$$

$$
\begin{aligned}
& \begin{array}{l}
\text { gave; } \\
\text { gave; }
\end{array} \quad\left[\begin{array}{l}
\varphi ; \\
x ; \\
\mathrm{NP} / \mathrm{PP} / \mathrm{NP}
\end{array}\right]^{1}
\end{aligned}
$$

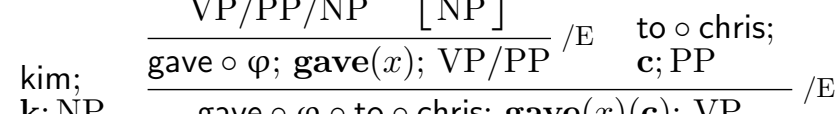

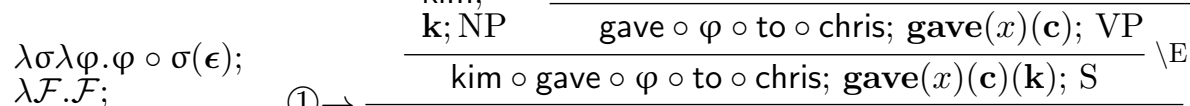

$$
\begin{aligned}
& \text { bagels; } \quad \frac{(\mathrm{S}\lceil X) \uparrow(\mathrm{S}\lceil X)}{\lambda \varphi . \varphi \circ \text { kim } \circ \text { gave } \circ \text { to } \circ \text { chris; } \lambda x \text { gave }(x)(\mathbf{c})(\mathbf{k}) ; \mathrm{S}\lceil\mathrm{NP}} \overline{\lambda(\mathbf{k}) ; \mathrm{S}\lceil\mathrm{NP}}\lceil\mathrm{I}
\end{aligned}
$$

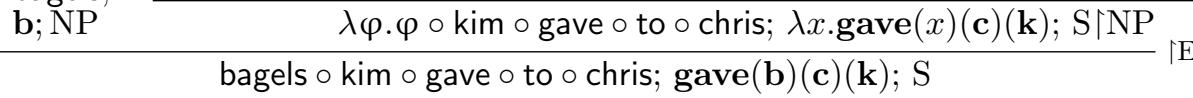

${ }^{13}$ One might worry about potential overgeneration of the following kind. Suppose we hypothesize the same variable in the two conjuncts of a conjoined sentence and bind them at once after the conjoined sentence is formed:

(i) $\lambda \varphi_{1} . \varphi_{1} \circ$ is $\circ$ male $\circ$ or $\circ \varphi_{1} \circ$ is $\circ$ female; $\lambda x . \operatorname{male}(x) \vee$ female $(x) ; \operatorname{S} \uparrow \mathrm{NP}$

Then, by combining this sign with the quantifier everyone, we seem to obtain the following:

(ii) everyone $\circ$ is $\circ$ male $\circ$ or $\circ$ everyone $\circ$ is $\circ$ female; $\mathbf{V}_{\text {person }}(\lambda x \cdot \operatorname{male}(x) \vee$ female $(x)) ; \mathrm{S}$

In other words, we (apparently) incorrectly predict that Everyone is male or everyone is female has the reading 'everyone is either male or female'.

Such a derivation actually doesn't go through. Recall from above that $\uparrow$ is linear, meaning that it can bind only one hypothesis at a time. This means that (i) can't be derived as a well-formed sign in Hybrid TLCG. For this reason, the above problematic derivation is correctly ruled out.

This of course raises the question of how to treat ATB extraction (see our analysis of 'overt movement' below):

(iii) John met a man who Mary likes _ but Sue hates _.

This is an important open question for the family of approaches including ours (as well as Muskens (2003) and Mihaliček and Pollard (2012)) in which the mode of implication ( $\uparrow$ in our calculus) used for overt movement is linear. Unfortunately, addressing this issue is beyond the scope of this paper, but see Morrill (2010) for some discussion about how multiple gaps (such as parasitic gaps) may be treated in a TLCG setup. 
In (41), a gapped sentence is derived just in the same way as in the quantifier example above via hypothetical reasoning for $\uparrow(1)$. The difference from the quantifier example is that the topicalization operator embeds an empty string $\epsilon$ to the gap position, thereby closing off the gap, and then concatenates the topicalized NP to the left of that string.

The analysis of covert movement (or quantifying-in) due to Oehrle (1994) illustrated above is not only theoretically illuminating, capturing the tight correlation between the semantic and phonological effects of quantification transparently, but it also has an empirical advantage over quantifying-in (and its analogs) as well. Specifically, this approach extends straightforwardly to more complex types of scope-taking, such as PARASITIC SCOPE in symmetrical predicates (Barker 2007; Pollard and Smith 2012) and SPLIT SCOPE of negative quantifiers (Kubota and Levine 2014a) and number determiners (Pollard 2014). Our own analysis of 'respective', symmetrical and summative predicates can be thought of as a further refinement of the parasitic scope analysis of symmetrical predicates by Barker (2007), and it exploits the flexible syntax-semantics interface enabled by the use of prosodic variable binding in the present approach. ${ }^{14}$ Further empirical motivation for the non-directional mode of implication comes from the analysis of Gapping (Kubota and Levine 2014a). Gapping is especially interesting in this connection in that it exhibits the properties of both 'overt' and 'covert' movement simultaneously, thus constituting a case that goes beyond the analytic possibilities available in the standard derivational architecture.

Finally, we would like to briefly comment on the relationship between Hybrid TLCG and related approaches in contemporary categorial grammar, namely, other variants of TLCG and Combinatory Categorial Grammar (CCG; Ades and Steedman 1982; Steedman 1996, 2000).

TLCG extends the Lambek calculus in various ways to cope with its empirical limitations (such as the medial extraction/quantification problem noted above). In the variants developed by Moortgat (1997), Bernardi (2002) and Vermaat (2005), this is achieved by employing a technique from substructural logic for recognizing different kinds of logic (each tied to distinct sets of 'structural' operations) simultaneously within a single calculus. The other line of work in TLCG, explored by Glyn Morrill and his colleagues (Morrill and Solias 1993, Morrill 1994 and Morrill et al. 2011, among others) recognizes a purpose-specific calculus for the prosodic component for treating discontinuity (in a broader sense encompassing extraction and quantification). While sharing the same general goals, we depart from these two traditions in an important way: our approach employs the standard $\lambda$-calculus for the prosodic component to deal with the discontinuity problem, thereby simplifying both the conceptual and technical foundations of TLCG considerably. Empirically, our calculus is most similar

\footnotetext{
${ }^{14}$ As noted by a reviewer, another possible extension of our analysis of 'respective' readings is binominal each, exemplified by the following sentence:

(i) The boys lifted three tables each.

The key problem that binominal each poses for compositional semantics is that even though each syntactically appears as a constituent attached to the NP designating the distributed share (three tables), it forces a distributive reading of the syntactically distant sorting key (the boys). By assuming that each takes a relation and two arguments corresponding to the sorting key and the distributive share as its syntactic arguments (this can be done by the same mechanism of double $\lambda$-abstraction in the syntax via the vertical slash that plays a key role in our analysis of 'respective' predication presented below), an analysis implementing Blaheta's (2003) strategy described in Dotlačil (2012) is in fact straightforward.
} 
to Barker's (2007) and Barker and Shan's (2015) $\mathrm{NL}_{\lambda} . \mathrm{NL}_{\lambda}$ is a version of the first type of TLCG described above utilizing structural postulates. The main differences between our calculus and $\mathrm{NL}_{\lambda}$ are that (i) $\mathrm{NL}_{\lambda}$ recognizes a novel structural postulate called the ' $\lambda$ ' postulate, whose conceptual and formal underpinnings are somewhat unclear (see Kubota (2010, 153, footnote 108) for some discussion on this point; but see also Barker and Shan (2015, Ch. 17) for a proof that $\mathrm{NL}_{\lambda}$ is equivalent to a slightly different calculus in which the ' $\lambda$ ' postulate is replaced by three distinct, 'perfectly kosher' structural rules in standard TLCG), whereas we achieve (more or less) the same result with a completely standard $\lambda$-calculus (but our approach is more powerful in recognizing higher-order variables in the prosodic component; cf. Kubota and Levine (2013b)); and (ii) $\mathrm{NL}_{\lambda}$, as its name suggests, is based on a non-associative variant of the Lambek calculus, whereas our system is associative (though this difference is perhaps not so critical, since, as Barker and Shan (2015) note, introducing associativity in their system is straightforward; likewise, restricting associativity in our calculus is also straightforward - see Kubota (2014) for a concrete proposal along these lines).

Unlike TLCG, CCG is notable for its strong thesis that the semantic interpretation of the sentence is directly obtained on the basis of the surface constituent structure (in the CCG sense, where 'nonconstituent' strings such as John met (in RNR) and Bill the book (in DCC) are taken to form constituents). There is thus no analog of the vertical slash in CCG. ${ }^{15}$ The treatment of scopal phenomena poses an obvious challenge for such an approach, but a recent proposal by Steedman (2012) attempts to meet this challenge by developing an analysis of quantification in English. However, there is currently no explicit analysis of 'respective' readings and related phenomena in CCG, and these phenomena are likely to present an even more difficult problem for it. The semantics of symmetrical predicates is known to be unexpressible in terms of generalized quantifiers (Keenan 1992). The coverage in Steedman (2012) is mostly limited to generalized quantifiers, and it is unclear whether the tightly constrained, strictly surface-oriented syntax-semantics interface of CCG is expressive enough to capture the properties of 'respective', symmetrical, and summative predicates in a fully general manner.

\subsection{The semantics of 'respective', symmetrical and summative predicates}

\subsubsection{Hypothetical reasoning and 'respective' predication}

We are now ready to analyze the three phenomena from Sect. 2. We start with the analysis of 'respective' readings since our analysis of the other two phenomena builds on the core semantic operator that we introduce for this construction. The underlying intuition of most formal analyses of 'respective' readings (cf. Gawron and Kehler 2004; Winter 1995; Bekki 2006) (which we also adopt) is that sentences like (42) involve pairwise predication between two (or more) sets of entities where the 'corresponding' elements of the two sets are related by some predicate in the sentence.

(42) Mary and Sue married John and Bill (respectively).

In the case of (42), this 'predicate' is simply the lexical meaning of the verb, but in more complex cases that we discuss below, the predicate that relates the elements of the two sets

\footnotetext{
${ }^{15}$ Another difference is that CCG directly posits rules like type raising and function composition (which are theorems in the Lambek calculus and Hybrid TLCG) as axioms.
} 
(as well as the elements in the two sets themselves) can be of a more elaborate type.

Among the previous approaches, Gawron and Kehler $(2004)(\mathrm{G} \& \mathrm{~K})$ work out the relevant compositional mechanism in most detail (see Sect. 4.1.1 for more on their approach). G\&K model the meanings of expressions to be related in a 'respective' manner in terms of the notion of sums. While this works well for cases of 'respective' readings, G\&K's approach faces a technical difficulty if one attempts to extend it directly to the case of symmetrical predicates. We refer the reader to Sect. 4.1.1 for details, but the issue essentially has to do with the fact that the sum of an identical atomic object $a \oplus a$ collapses to the same object $a$ in G\&K's setup. Since our goal is to provide a unified analysis of the three phenomena, we reformulate their analysis to fix this technical problem. The revision we introduce involves taking the denotations of plural and conjoined expressions as multisets rather than sets (as in G\&K's original formulation). The rest of the analysis, including the crucial use of the sequencing function (explained below) to retrieve the order of elements of the multiset in reference to (either linguistically or pragmatically determined) contextual information remains the same as in G\&K's original analysis.

Multisets are different from sets in that multiple occurrences of the same item makes a difference. Thus, by taking $A=\{a, a, b\}$ and $B=\{a, b\}, A$ and $B$ are the same set but different multisets. Since we do not use (non-multi-)sets for any purpose in our formal analysis, from now on we use the curly brace notation only for writing multisets. Thus, the reader should note that in the rest of the present paper, it is taken for granted that $\{a, a, b\} \neq\{a, b\}$, etc. We define the cardinality of multisets as the number of elements that the multiset contains, counting the multiple occurrences of the same element distinctly. Thus, the cardinality of $A=\{a, a, b\}$ is $|A|=3$ and this is distinct from the cardinality of $B=\{a, b\},|B|=2$.

We now recast G\&K's analysis of 'respective' readings in this multiset-based setup. First, we assume that, for any semantic type $\tau$, the conjunction word and denotes the following multiset-forming operator that forms a multiset of objects of type $\tau$ containing the denotations of each conjunct as distinct elements of the multiset formed (we omit the details, but generalizing this entry to cases involving more than two conjuncts is straightforward):

$$
\text { and; } \lambda \mathcal{W} \lambda \mathcal{V} \cdot\{\mathcal{V}, \mathcal{W}\} ;(X \backslash X) / X
$$

This enables us to assign multisets of individuals like $\{$ mary, sue $\}$ and $\{$ mary, sue, ann $\}$ as the meanings of expressions like Mary and Sue and Mary, Sue and Ann. Then, to assign the right meaning to (42), the two multisets $\{$ mary, sue $\}$ and $\{$ john, bill $\}$, each denoted by the subject and object NPs, need to be related to each other in a 'respective' manner via the relation married: Mary married John and Sue married Bill.

Establishing this 'respective' relation is mediated by the resp operator (defined in (44) below), which is a prosodically empty operator that takes a relation and two multiset-denoting terms as arguments, and returns a multiset consisting of propositions obtained by relating each member of the two multisets in a pairwise manner with respect to the relation in question. As in G\&K's analysis, in establishing the 'respective' relation between the two multisets, the resp operator crucially makes reference to the sequencing function $f_{\text {seq }}$, which is a function that takes an integer $i$ and a multiset $X_{\mu}$ as arguments and returns the $i$ th member of $X_{\mu}$ based on some contextually established ordering of elements of $X_{\mu}$. In most cases involving overt conjunction, the relevant ordering is linguistically given: it simply corresponds to the linear 
order of conjuncts in the conjunction. On this assumption, for the multiset $A=\{\mathbf{j o h n}, \mathbf{b i l l}\}$ denoted by the conjunction John and Bill, $f_{\text {seq }}(1)(A)=$ john and $f_{\text {seq }}(2)(A)=$ bill.

The resp operator is defined as follows:

$$
\operatorname{resp}=\lambda \mathcal{R} \lambda \mathcal{T}_{\mu_{n}} \lambda \mathcal{U}_{\mu_{n}} \cdot\left\{V \mid \exists i .1 \leq i \leq n \wedge V=\mathcal{R}\left(f_{\text {seq }}(i)\left(\mathcal{T}_{\mu_{n}}\right)\right)\left(f_{\text {seq }}(i)\left(\mathcal{U}_{\mu_{n}}\right)\right)\right\}
$$

$\mathcal{T}_{\mu_{n}}$ and $\mathcal{U}_{\mu_{n}}$ are (polymorphic) variables over multisets with cardinality $n$ (we omit the subscript $n$ if the cardinality is obvious from the context). Thus, in (44), the cardinality of the two input multisets are required to match.

For each $i$ such that $1 \leq i \leq n$ (where $n$ is the cardinality of the input multisets), the resp operator relates the $i$ th members of the multisets denoted by its second and third arguments via the relation that it takes as its first argument to form a proposition $\mathcal{R}\left(f_{\text {seq }}(i)\left(\mathcal{T}_{\mu_{n}}\right)\right)\left(f_{\text {seq }}(i)\left(\mathcal{U}_{\mu_{n}}\right)\right)$. The output of the resp operator is simply a multiset consisting of all such propositions. Since $i$ ranges over the cardinality of the input multisets in (44), the cardinality of the output multiset matches that of the input multisets.

By giving the relation denoted by the verb and the two multisets denoted by the subject and object NPs as arguments to the resp operator, we obtain the translation in (45), which can be simplified as in (46) on the assumption that $f_{\text {seq }}$ corresponds to the ordering reflecting the surface order of conjuncts (we take $f_{\text {say }}$ to be a specific instance of the ordering function $f_{\text {seq }}$ that is sensitive to surface word order; thus another way to put it is that the simplification in (46) goes through iff $f_{\text {seq }}=f_{\text {say }}$ ). We write the first argument of $f_{\text {seq }}$ as a superscript, to make the notation easier to read.

$$
\begin{aligned}
& \operatorname{resp}(\operatorname{married})(\{\mathbf{j}, \mathbf{b}\})(\{\mathbf{m}, \mathbf{s}\}) \\
& =\left\{\operatorname{married}\left(f_{\text {seq }}^{1}(\{\mathbf{j}, \mathbf{b}\})\right)\left(f_{\text {seq }}^{1}(\{\mathbf{m}, \mathbf{s}\})\right), \operatorname{married}\left(f_{\text {seq }}^{2}(\{\mathbf{j}, \mathbf{b}\})\right)\left(f_{\text {seq }}^{2}(\{\mathbf{m}, \mathbf{s}\})\right)\right\} \\
& =\{\operatorname{married}(\mathbf{j})(\mathbf{m}), \operatorname{married}(\mathbf{b})(\mathbf{s})\}
\end{aligned}
$$

This multiset consisting of two propositions is then mapped to a boolean conjunction via a phonologically empty operator with the following meaning:

$$
\lambda p_{\mu} \cdot \bigwedge_{\mu} p_{\mu}
$$

Here, $\bigwedge_{\mu}$ is the boolean conjunction operator for multisets, which returns the boolean conjunction of all the elements of the multiset that it takes as an argument. For example, $\bigwedge_{\mu}\{a, a, b\}=a \wedge b$. By applying (47) to (46), we obtain the proposition $\operatorname{married}(\mathbf{j})(\mathbf{m}) \wedge$ $\operatorname{married}(\mathbf{b})(\mathbf{s})$. As will become clear below, keeping the two components separate in the form of a multiset after the application of the resp operator is crucial for dealing with multiple 'respective' (or symmetrical/summative) readings in examples like those in (9), (13) and (18).

The next question is how to get this semantic analysis hooked up with a compositional analysis of the sentence. Things may seem simple and straightforward in examples like (42), where the two terms to be related to each other in a pairwise manner are co-arguments of the same predicate. However, as noted already, this is not always the case. For treating more complex cases, G\&K propose to treat 'respective' predication in terms of a combination of recursive applications of both the 'respective' operator and the distributive operator, but their approach quickly becomes unwieldy. Since we need to deal with complex examples involving 
interactions with nonconstituent coordination, we simply note here that the compositional mechanism assumed by G\&K is not fully general and turn to an alternative approach (see Sect. 4.1.1 for a more complete critique of their approach; see also Sect. 5 for some more general remarks about the relationship between the present proposal and G\&K's approach).

It turns out that a more general (and simpler) approach which serves our purpose here is straightforwardly available in Hybrid TLCG, by (two instances of) hypothetical reasoning via the vertical slash, as we show momentarily. Crucially, the interdependence between the two multisets is mediated by double abstraction via $\lceil$ in the syntax, whose output (together with the two multisets themselves) is immediately given to the resp operator, which then relates them in a pairwise manner with respect to some relation $\mathcal{R}$. This is essentially an implementation of Barker's (2007) 'parasitic scope' strategy. (For a comparison between the present proposal and Barker's analysis of same, see Sect. 4.1.2.)

In Hybrid TLCG, we can abstract over any arbitrary positions in a sentence to create a relation that obtains between objects belonging to the semantic types of the variables that are abstracted over. This is illustrated in the following partial derivation for (42). By abstracting over the subject and object positions of the sentence, we obtain an expression of type $\mathrm{S} \backslash \mathrm{NP} \backslash \mathrm{NP}$, where the 'gaps' in the subject and object positions are kept track of via explicit $\lambda$-binding in the phonology, just in the same way as in the analysis of quantifier scope above (here and elsewhere, VP abbreviates NP $\backslash \mathrm{S}$ ).

$$
\begin{aligned}
& \underline{\left[\varphi_{2} ; y ; \mathrm{NP}\right]^{2}} \frac{\text { married; married; VP/NP }\left[\varphi_{1} ; x ; \mathrm{NP}\right]^{1}}{\operatorname{married} \circ \varphi_{1} ; \operatorname{married}(x) ; \mathrm{VP}} / \mathrm{E} \\
& \varphi_{2} \circ \text { married } \circ \varphi_{1} ; \operatorname{married}(x)(y) ; \mathrm{S}
\end{aligned}
$$

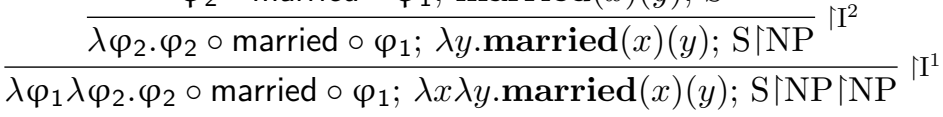

The 'respective' operator, defined as in (49), then takes such a doubly-abstracted proposition as an argument to produce another type $\mathrm{S}\rceil \mathrm{NP}\lceil\mathrm{NP}$ expression. Phonologically, it is just an identify function, and its semantic contribution is precisely the resp operator defined above.

$$
\lambda \sigma \lambda \varphi_{1} \lambda \varphi_{2} . \sigma\left(\varphi_{1}\right)\left(\varphi_{2}\right) ; \operatorname{resp} ;(Z|X| Y) \uparrow(Z|X| Y)
$$

The derivation completes by giving the two multisets denoted by John and Bill and Mary and Sue to this 'respectivized' type $\mathrm{S} \backslash \mathrm{NP} \backslash \mathrm{NP}$ predicate, and converting the pair of propositions to a boolean conjunction by the boolean reduction operator in (47).

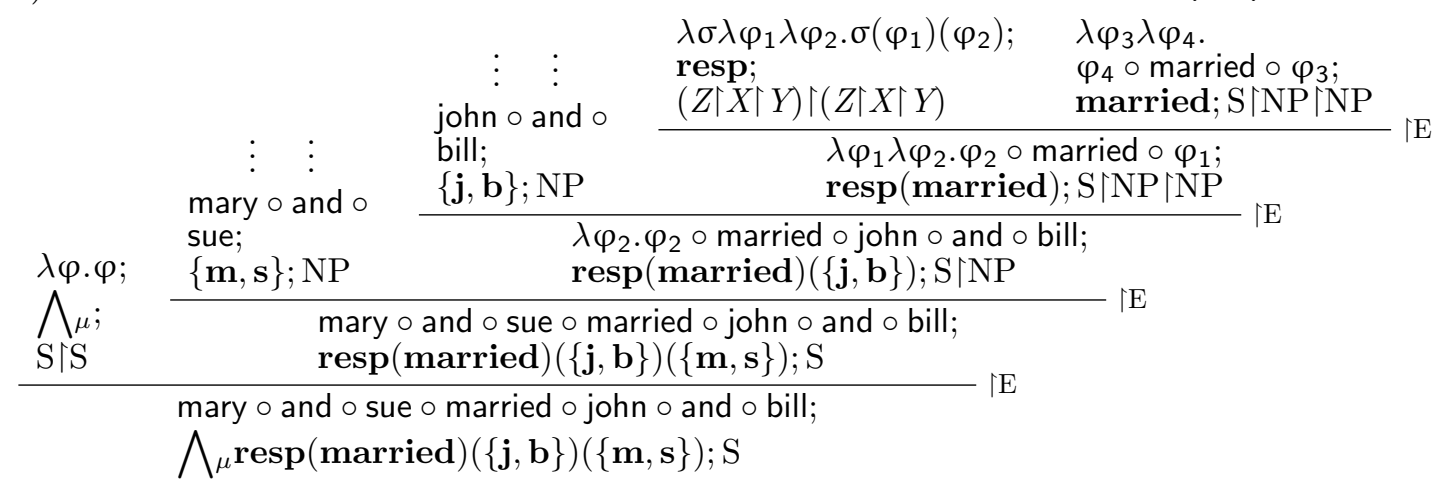


As noted above, by assuming $f_{\text {seq }}=f_{\text {say }}$, the final translation reduces to the boolean conjunction $\operatorname{married}(\mathbf{j})(\mathbf{m}) \wedge \operatorname{married}(\mathbf{b})(\mathbf{s})$. In what follows, we implicitly make this assumption in unpacking and simplifying translations, since except for special cases (discussed in Sect. 4.2), it is safe to assume that $f_{\text {seq }}=f_{\text {say }}$ does indeed hold.

Note here that in (50), prosodic $\lambda$-binding with $\lceil$ enables 'lowering' the phonologies of the two multiset-denoting expressions in their respective positions in the sentence, thus mediating the syntax-semantics mismatch between their surface positions and semantic scope (of the resp operator that they are arguments of) in essentially the same way as with quantifiers.

We would like to briefly comment on the treatment of the adverb respectively at this point. We assume that respectively simply denotes a version of the resp operator in which $f_{\text {seq }}$ is fixed to $f_{\text {say }}$, which picks up elements of a multiset simply based on the order of mention: ${ }^{16}$

$$
\begin{aligned}
& \lambda \sigma \lambda \varphi_{1} \lambda \varphi_{2} . \sigma\left(\varphi_{1}\right)\left(\varphi_{2}\right) \circ \text { respectively; } \\
& \lambda \mathcal{R} \lambda \mathcal{T}_{\mu_{n}} \lambda \mathcal{U}_{\mu_{n}} .\left\{V \mid \exists i .1 \leq i \leq n \wedge V=\mathcal{R}\left(f_{\text {say }}(i)\left(\mathcal{T}_{\mu_{n}}\right)\right)\left(f_{\text {say }}(i)\left(\mathcal{U}_{\mu_{n}}\right)\right)\right\} ;(Z|X| Y)\lceil(Z|X| Y)
\end{aligned}
$$

One point to note about respectively is that it is an adverb, and just like other adverbs, its surface word order is relatively flexible.
a. John and Mary will meet Peter and Sue, respectively.
b. John and Mary respectively will meet Peter and Sue.
c. John and Mary will respectively meet Peter and Sue.

With the lexical entry in (51), our analysis attaches respectively at the end of the whole string (corresponding to the word order in (52a)). We assume that surface reordering principles (which can be implemented readily by adopting a multi-modal version of TLCG such as Moortgat and Oehrle (1994), Muskens (2007) and Kubota (2014)) are responsible for generating the other orders such as $(52 \mathrm{~b}, \mathrm{c})$.

The present analysis extends straightforwardly to cases in which one of the multisetdenoting terms appears in a sentence-internal position, such as the following:

(53) John and Bill sent the bomb and the letter to the president yesterday, respectively.

For this sentence, we first obtain the following doubly abstracted proposition in the same way as in the simpler example above:

(54) $\lambda \varphi_{1} \lambda \varphi_{2} \cdot \varphi_{1} \circ$ sent $\circ \varphi_{2} \circ$ to $\circ$ the $\circ$ president $\circ$ yesterday; $\lambda x \lambda y \cdot \operatorname{yest}(\operatorname{sent}(y)($ the-pres $))(x) ;$ $\mathrm{S}\lceil\mathrm{NP}\lceil\mathrm{NP}$

The resp operator then takes this and the two multiset-denoting terms as arguments to produce a sign with the surface string in (53) paired with the following semantic interpretation (after the application of boolean reduction):

\footnotetext{
${ }^{16}$ This analysis predicts that the contribution of respectively is truth-conditional. This seems to be the correct result. Note that the relevant content can be directly negated (similarly, examples can be readily constructed to show that the meaning contribution of respectively scopes under standard presupposition/CI holes such as conditionals and modals).
}

(i) A: Did John and Bill meet Mary and Sue respectively?

B: No - even though John met Sue and Bill met Mary, John didn't meet Mary or Bill Sue. 


$$
\operatorname{yest}(\operatorname{sent}(\text { the-bomb })(\text { the-pres }))(\mathbf{j}) \wedge \operatorname{yest}(\operatorname{sent}(\text { the-letter })(\text { the-pres }))(b)
$$

We now turn to an interaction with NCC, taking the following example as an illustration:

(56) John and Bill met Robin on Thursday and Chris on Friday, respectively.

The analysis is in fact straightforward. As discussed in Sect. 3.1.1, in TLCG, dependent cluster coordination is analyzed by treating the apparent nonconstituents that are coordinated in examples like (56) to be (higher-order) derived constituents, via hypothetical reasoning (with the directional slashes / and $\backslash$ ).

Specifically, via hypothetical reasoning, the string Robin on Thursday can be analyzed as a constituent of type $(\mathrm{VP} / \mathrm{NP}) \backslash \mathrm{VP}$, an expression that combines with a transitive verb and an NP (in that order) to its left to become an S (see (116) in Appendix A for a complete proof):

$$
\text { robin } \circ \text { on } \circ \text { thursday; } \lambda R \text {.on } \mathbf{T h}(R(\mathbf{r})) ;(\mathrm{VP} / \mathrm{NP}) \backslash \mathrm{VP}
$$

We then derive a sentence containing gap positions corresponding to this derived constituent and the subject NP ((117) in Appendix A):

$$
\lambda \varphi_{1} \lambda \varphi_{2} . \varphi_{1} \circ \text { met } \circ \varphi_{2} ; \lambda x \lambda \mathscr{P} . \mathscr{P}(\text { met })(x) ; \mathrm{S} \uparrow((\mathrm{VP} / \mathrm{NP}) \backslash \mathrm{VP})\lceil\mathrm{NP}
$$

The rest of the derivation involves giving this relation and the two multiset-denoting arguments of types $\mathrm{NP}$ and $(\mathrm{VP} / \mathrm{NP}) \backslash \mathrm{VP}$ as arguments to the resp operator, which yields the following translation for the whole sentence:

$$
\operatorname{onTh}(\operatorname{met}(\mathbf{r}))(\mathbf{j}) \wedge \operatorname{onFr}(\operatorname{met}(\mathbf{c}))(\mathbf{b})
$$

Finally, multiple 'respective' readings, exemplified by (60), is straightforward.

(60) Tolstoy and Dostoevsky sent Anna Karenina and The Idiot to Dickens and Thackeray (respectively).

As in G\&K's analysis, the right meaning can be compositionally assigned to the sentence via recursive application of the resp operator, without any additional mechanism. The key point of the derivation is that we first derive a three-place predicate of type $\mathrm{S}\lceil\mathrm{NP}\lceil\mathrm{NP}\lceil\mathrm{NP}$, instead of a two-place predicate of type $\mathrm{S}\lceil\mathrm{NP}\lceil\mathrm{NP}$ (as in the simpler case in (50) above), to be given as an argument to the first resp operator:

\section{(61) $\lambda \varphi_{1} \lambda \varphi_{2} \lambda \varphi_{3} . \varphi_{3} \circ$ sent $\circ \varphi_{1} \circ$ to $\circ \varphi_{2} ;$ sent; $\mathrm{S}\lceil\mathrm{NP}\lceil\mathrm{NP}\lceil\mathrm{NP}$}

After two of the multiset-denoting terms are related to each other with respect to the predicate denoted by the verb, the resultant $\mathrm{S} \backslash \mathrm{NP}$ expression denotes a multiset consisting of two properties (note that the semantic translation in (62) can be simplified as $\{\mathbf{s e n t}(\mathbf{a k})(\mathbf{d i}), \mathbf{s e n t}(\mathbf{i d})(\mathbf{t h})\}$ assuming $f_{\text {seq }}=f_{\text {say }}$; also, see (118) in Appendix A for a complete proof):

$$
\lambda \varphi_{3} . \varphi_{3} \circ \text { sent } \circ \mathrm{AK} \circ \text { and } \circ \mathrm{Id} \circ \text { to } \circ \mathrm{Di} \circ \text { and } \circ \mathrm{Th} ; \boldsymbol{r e s p}(\operatorname{sent})(\{\mathbf{a k}, \mathbf{i d}\})(\{\mathbf{d i}, \mathbf{t h}\}) ; \mathrm{S} \backslash \mathrm{NP}
$$

And the remaining conjoined term $\{\mathbf{t o}, \mathbf{d o}\}$ is related to this property multiset by a derived two-place 'respective' operator in the following way: 


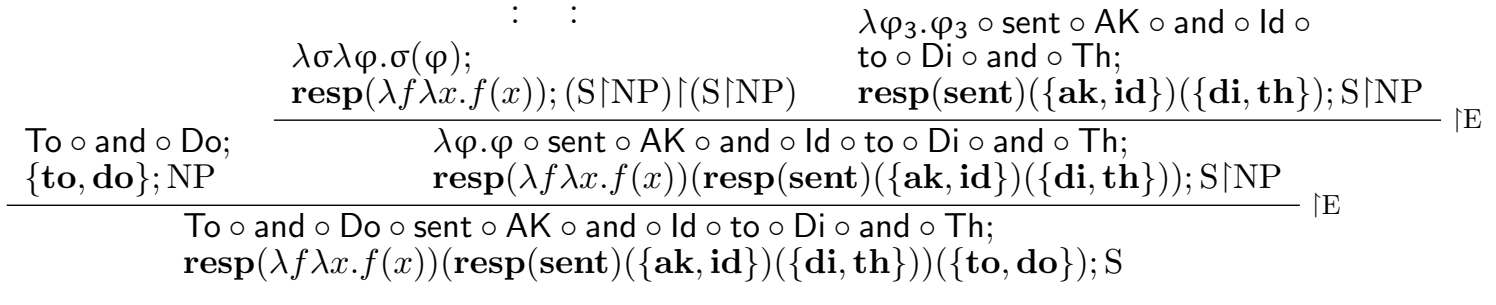

The final translation obtained in (63) can be unpacked and simplified as follows (again, assuming $\left.f_{\text {seq }}=f_{\text {say }}\right)$ :

$$
\begin{aligned}
\operatorname{resp} & (\lambda f \lambda x \cdot f(x))(\operatorname{resp}(\mathbf{s e n t})(\{\mathbf{a k}, \mathbf{i d}\})(\{\mathbf{d i}, \mathbf{t h}\}))(\{\mathbf{t o}, \mathbf{d o}\}) \\
\quad= & \operatorname{resp}(\lambda f \lambda x \cdot f(x))(\{\operatorname{sent}(\mathbf{a k})(\mathbf{d i}), \operatorname{sent}(\mathbf{i d})(\mathbf{t h})\})(\{\mathbf{t o}, \mathbf{d o}\}) \\
\quad= & \{\operatorname{sent}(\mathbf{a k})(\mathbf{d i})(\mathbf{t o}), \operatorname{sent}(\mathbf{i d})(\mathbf{t h})(\mathbf{d o})\}
\end{aligned}
$$

The two-place resp operator, which directly relates the property multiset (of type $\mathrm{S}\lceil\mathrm{NP}$ ) with the NP multiset occupying the subject position via pairwise function application of the corresponding elements, can be derived as a theorem from the lexically specified three-place resp operator via hypothetical reasoning. The proof is given in (119) in Appendix A.

Before moving on, we would like to address one important issue regarding the meaning of the conjunction word and. We have assumed above that and uniformly denotes the multisetforming operator in (43) for any semantic type. The reader might wonder at this point whether this is the only meaning of conjunction that we need or we additionally need to assume the boolean conjunction meaning (and generalize it also to non-propositional types along the lines of Partee and Rooth (1983)). ${ }^{17}$ As discussed in Kubota and Levine (2014d) (building on Bekki (2006)), the two rules in (115) in Sect. 5 become derivable in our setup. We assume that sentences like (47a) and the distributive readings of sentences like (47b) are obtained with these rules, and that boolean conjunction does not need to be separately posited.
a. John walked and talked.
b. John and Bill walked.

For example, (65a) can be derived by taking the VP walked and talked to denote the multiset $\{$ walk, talk $\}$ and by combining the VP and the subject NP via rule (115b) (more precisely, a variant of rule (115b) in which the functor category is $B \backslash A$ instead of $A / B$, which is also derivable as a theorem).

\subsubsection{Extending the analysis to symmetrical and summative predicates}

We exploit the resp operator introduced above in the analysis of symmetrical and summative predicates as well. The intuition behind this approach is that NPs containing same, different, etc. (we call such NPs 'symmetrical terms' below) in examples like (66) denote multisets (linked to the other multiset denoted by the plural John and Bill in the same way as in the 'respective' readings above) but that they impose special conditions on each element of the multiset.

\footnotetext{
${ }^{17}$ See also Krifka (1990) for some discussion about the relationship between boolean and non-boolean and.
} 
John and Bill read the same book.

In (66), John and Bill need to be each paired with an identical book, and in the case of different, they need to be paired with distinct books. To capture this additional constraint on the multisets denoted by symmetrical terms, we assign to them GQ-type meanings of type $\mathrm{S} \uparrow(\mathrm{S}\lceil\mathrm{NP})$, where the abstracted NP in their arguments are multiset-denoting expressions semantically. More specifically, we posit the following lexical entries for the same and different: ${ }^{18}$

$$
\begin{array}{ll}
\text { a. } & \lambda \varphi \lambda \sigma \cdot \sigma(\text { the } \circ \text { same } \circ \varphi) ; \\
& \lambda P \lambda Q . \exists X_{\mu} \forall i \in X_{\mu} . P(i) \wedge \forall i, j \in X_{\mu}[i=j] \wedge Q\left(X_{\mu}\right) ; \mathrm{S}\lceil(\mathrm{S}\lceil\mathrm{NP}) \uparrow \mathrm{N} \\
\text { b. } \quad \lambda \varphi \lambda \sigma . \sigma(\text { different } \circ \varphi) ; \\
& \lambda P \lambda Q . \exists X_{\mu} \forall i \in X_{\mu} . P(i) \wedge \forall i, j \in X_{\mu}\left[f_{\text {seq }}^{i} \neq f_{\text {seq }}^{j} \rightarrow i \neq j\right] \wedge Q\left(X_{\mu}\right) ; \mathrm{S}\lceil(\mathrm{S}\lceil\mathrm{NP})\lceil\mathrm{N}
\end{array}
$$

In both cases, the relevant multiset (which enters into the 'respective' relation with another multiset via the resp operator) consists of objects that satisfy the description provided by the noun. The difference is that in the case of same, the elements of the multiset are all constrained to be identical, whereas in the case of different, they are constrained to differ from one another.

We now outline the analysis for (66) (the full derivation is given in (120) in Appendix A). The key point is that we first posit a variable that semantically denotes a multiset and relate it with the other multiset-denoting expression (John and Bill in this case) via the resp operator. This part of the analysis follows proof steps completely parallel to the analysis of 'respective' readings shown in the previous section. Specifically, by hypothetically assuming an NP with phonology $\varphi$ and semantics $Z_{\mu}$, we can derive the expression in (68).

$$
\text { john } \circ \text { and } \circ \text { bill } \circ \text { read } \circ \varphi ; \bigwedge \mu \operatorname{resp}(\operatorname{read})\left(Z_{\mu}\right)(\{\mathbf{j}, \mathbf{b}\}) ; \mathrm{S}
$$

At this point (where boolean reduction has already taken place), we withdraw the hypothesis to obtain an expression of type $\mathrm{S}\lceil\mathrm{NP}$. This is then given as an argument to the symmetrical term the same book, which, as noted above, has the GQ type category $\mathrm{S} \uparrow(\mathrm{S} \uparrow \mathrm{NP})$. The symmetrical term lowers its phonology to the gap and semantically imposes the identity condition on the members of the relevant multiset. These last steps are illustrated in (69).

\footnotetext{
${ }^{18}$ There is a close connection between the lexical entries for the internal readings posited in (67) and those for the external readings. The lexical entries in (67) essentially establish (non-)identity among each element of a multiset, and in this sense, it can be thought of as involving a reflexive anaphoric reference. By replacing this reflexive anaphoric reference with an anaphoric reference to some external object and stating the (non-)identity conditions to pertain to the object identified by the symmetrical term and the anaphorically invoked external object, we obtain a suitable lexical meaning for the external readings for same and different. Thus, while it may not be possible to unify the lexical entries for the two readings completely, we believe that our approach provides a basis for understanding the close relationship between the two readings. In fact, whether a unified analysis of internal and external readings is desirable seems still controversial. See Brasoveanu (2011) and Bumford and Barker (2013) for some recent discussion.
} 


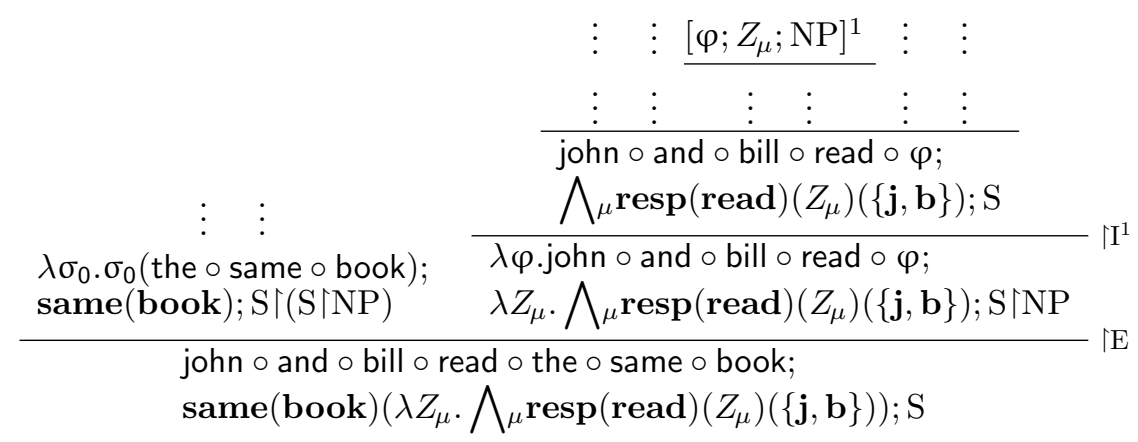

The reason that boolean reduction precedes the abstraction over the multiset-denoting NP in $(69) /(120)$ is that the meaning of the symmetrical term is a GQ over multiset-denoting NPs. That is, the type S「NP property given to it as an argument is a property of multiset-denoting NPs that returns a truth value, rather than a multiset, by taking its multiset-denoting NP argument. One way to see what's going on in the derivation in (69) is that the hypothetical reasoning with the multiset-denoting variable $Z_{\mu}$ and 'respective' predication involving it is needed since a symmetrical term denotes a quantifier over multiset-denoting expressions and hence cannot directly fill in an argument slot of a lexical verb (which is looking for a non-multiset-denoting expressions as its arguments).

The final translation is unpacked in (70) (again, assuming $f_{\text {seq }}=f_{\text {say }}$ for the subject NP John and Bill):

$$
\begin{aligned}
& \text { same }(\mathbf{b o o k})\left(\lambda X_{\mu} \cdot \bigwedge{ }_{\mu} \operatorname{resp}(\operatorname{read})\left(X_{\mu}\right)(\{\mathbf{j}, \mathbf{b}\})\right) \\
& =\exists X_{\mu} \forall i \in X_{\mu} \cdot \operatorname{book}(i) \wedge \forall i, j \in X_{\mu}[i=j] \wedge \bigwedge_{\mu} \operatorname{resp}(\operatorname{read})\left(X_{\mu}\right)(\{\mathbf{j}, \mathbf{b}\}) \\
& =\exists X_{\mu} \forall i \in X_{\mu} \cdot \operatorname{book}(i) \wedge \forall i, j \in X_{\mu}[i=j] \wedge \operatorname{read}\left(f_{\text {seq }}^{1}\left(X_{\mu}\right)\right)(\mathbf{j}) \wedge \operatorname{read}\left(f_{\text {seq }}^{2}\left(X_{\mu}\right)\right)(\mathbf{b})
\end{aligned}
$$

Since $f_{\text {seq }}^{1}\left(X_{\mu}\right)=f_{\text {seq }}^{2}\left(X_{\mu}\right)$, this correctly ensures that the book that John read and the one that Bill read are identical.

Before moving on to more complex cases, a comment is in order on the semantic analysis of same and different in (67). So far as we can tell, the lexical meanings given in (67) capture the truth conditions for the internal readings of same and different correctly. One might think that $(67 \mathrm{a})$ is too weak as the meaning of same since according to this definition, (66) can be true in a situation in which the sets of books that John and Bill read are different, as long as one can identify some common book read by both individuals. Thus, one might think that some kind of maximality condition should be imposed on the set of books identified by same. We believe that this maximality condition, which does indeed seem to be present in most ordinary contexts in which sentences like (66) are uttered, is an implicature associated with the sentence rather than part of its truth conditions. Note that (66) is true and felicitous as long as one can identify (at least) one book commonly read by John and Bill. They may have read other books in addition, but that doesn't make (66) false or infelicitous.

(71) John and Bill read the same book, although they both read several different books in addition.

Similarly, (67b), as it stands, does not exclude a possibility in which there is some set of books commonly read by John and Bill. We again take this to be the correct result. The 
following example shows that the implication excluding the existence of common books read by the two (which indeed seems to be typically present) is not part of the entailment of the sentence: ${ }^{19}$

(72) John and Bill read different books, although they read the same books too.

We now move on to multiple dependency cases. In fact, the present analysis already assigns the right meanings to these sentences. Specifically, since the same resp operator is at the core of the analysis as in the case of 'respective' readings, we immediately predict that symmetrical predicates can enter into multiple dependencies both among themselves and with respect to 'respective' predication, as exemplified by examples like the following:

a. John and Bill gave the same book to Mary and Sue (respectively).

b. John and Bill gave the same book to the same man.

Since the relevant derivations can be reconstructed by taking the derivation for multiple 'respective' readings presented in the previous section as a model, we omit the details and reproduce here only the derived meanings for (73a) and (73b) in (74) and (75), respectively (see (121) in Appendix A for a complete derivation for (73b)).

$$
\begin{aligned}
& \text { same }(\mathbf{b o o k})\left(\lambda X_{\mu} \cdot \bigwedge{ }_{\mu} \operatorname{resp}(\lambda f \lambda x \cdot f(x))\left(\operatorname{resp}(\lambda x \lambda y \lambda w \cdot \operatorname{gave}(x)(w)(y))\left(X_{\mu}\right)(\{\mathbf{j}, \mathbf{b}\})\right)(\{\mathbf{m}, \mathbf{s}\})\right) \\
& =\operatorname{same}(\mathbf{b o o k})\left(\lambda X_{\mu} \cdot \operatorname{gave}(\mathbf{m})\left(f_{\text {seq }}^{1}\left(X_{\mu}\right)\right)(\mathbf{j}) \wedge \operatorname{gave}(\mathbf{s})\left(f_{\text {seq }}^{2}\left(X_{\mu}\right)\right)(\mathbf{b})\right) \\
& =\exists X_{\mu} \forall i \in X_{\mu} \cdot \mathbf{b o o k}(i) \wedge \forall i, j \in X_{\mu}[i=j] \wedge \operatorname{gave}(\mathbf{m})\left(f_{\text {seq }}^{1}\left(X_{\mu}\right)\right)(\mathbf{j}) \wedge \operatorname{gave}(\mathbf{s})\left(f_{\text {seq }}^{2}\left(X_{\mu}\right)\right)(\mathbf{b}) \\
& \text { same }(\mathbf{b o o k})\left(\lambda X_{\mu} \cdot \operatorname{same}(\mathbf{m a n})\right. \\
& \left.\quad\left(\lambda Y_{\mu} \cdot \bigwedge{ }_{\mu} \operatorname{resp}(\lambda f \lambda x \cdot f(x))\left(\operatorname{resp}(\lambda x \lambda y \lambda w \cdot \operatorname{gave}(x)(w)(y))\left(X_{\mu}\right)(\{\mathbf{j}, \mathbf{b}\})\right)\left(Y_{\mu}\right)\right)\right) \\
& =\operatorname{same}(\mathbf{b o o k})\left(\lambda X_{\mu} \cdot \operatorname{same}(\mathbf{m a n})\left(\lambda Y_{\mu} \cdot \operatorname{gave}\left(f_{\text {seq }}^{1}\left(Y_{\mu}\right)\right)\left(f_{\text {seq }}^{1}\left(X_{\mu}\right)\right)(\mathbf{j}) \wedge \operatorname{gave}\left(f_{\text {seq }}^{2}\left(Y_{\mu}\right)\right)\left(f_{\text {seq }}^{2}\left(X_{\mu}\right)\right)(\mathbf{b})\right)\right) \\
& =\exists X_{\mu} \forall i \in X_{\mu} \cdot \mathbf{b o o k}(i) \wedge \forall i, j \in X_{\mu}[i=j] \wedge \exists Y_{\mu} \forall i \in Y_{\mu} \cdot \mathbf{m a n}(i) \wedge \forall i, j \in Y_{\mu}[i=j] \wedge \\
& \quad \quad \operatorname{gave}\left(f_{\text {seq }}^{1}\left(Y_{\mu}\right)\right)\left(f_{\text {seq }}^{1}\left(X_{\mu}\right)\right)(\mathbf{j}) \wedge \operatorname{gave}\left(f_{\text {seq }}^{2}\left(Y_{\mu}\right)\right)\left(f_{\text {seq }}^{2}\left(X_{\mu}\right)\right)(\mathbf{b})
\end{aligned}
$$

The derivation for the multiple same sentence (73b) involves first positing two multisetdenoting variables $X_{\mu}$ and $Y_{\mu}$, which are linked to the plural term John and Bill via the recursive application of the resp operator and then bound by the two GQs over multisetdenoting terms the same man and the same book.

In the present analysis, the interaction between multiple 'respective' predication with NCC, exemplified by sentences like the following, is similarly straightforward:

(76) Terry gave the same gift to Bill and Sue as a Christmas present on Thursday and as a New Year's gift on Saturday (respectively).

The full derivation, which combines the proof steps for the NCC/'respective' interaction and multiple 'respective' readings already outlined, is given in (122) in Appendix A. We reproduce here the final translation and unpack it:

\footnotetext{
${ }^{19}$ There is a certain awkwardness to $(72)$. But again, we believe that this arises from a Gricean implicature. Had the speaker known that (72) were the case, s/he could have instead more cooperatively said John and Bill read some of the same books, but some different ones too, or something equivalent. Thus, we take (72) to be only awkward, but crucially, not contradictory.
} 


$$
\begin{aligned}
& \operatorname{same}(\operatorname{gift})\left(\lambda X_{\mu} \cdot \bigwedge{ }_{\mu} \operatorname{resp}(\lambda f \lambda x . f(x))\left(\operatorname{resp}(\lambda x \lambda y \lambda \mathscr{F} . \mathscr{F}(\operatorname{gave}(x)(y))(\mathbf{t}))\left(X_{\mu}\right)(\{\mathbf{b}, \mathbf{s}\})\right)\right. \\
& \quad(\{\lambda P . \operatorname{onTh}(\operatorname{asChP}(P)), \lambda P . \operatorname{onSat}(\operatorname{asNYG}(P))\})) \\
& =\operatorname{same}(\operatorname{gift})\left(\lambda X_{\mu} \cdot \mathbf{o n T h}\left(\operatorname{asChP}\left(\operatorname{gave}\left(f_{\text {seq }}^{1}\left(X_{\mu}\right)(\mathbf{b})\right)\right)\right)(\mathbf{t}) \wedge \operatorname{onSat}\left(\operatorname{asNYG}\left(\operatorname{gave}\left(f_{\text {seq }}^{2}\left(X_{\mu}\right)(\mathbf{s})\right)\right)\right)(\mathbf{t})\right) \\
& =\exists X_{\mu} \cdot \forall i \in X_{\mu} \cdot \operatorname{gift}(i) \wedge \forall i, j \in X_{\mu}[i=j] \wedge \\
& \quad \operatorname{onTh}\left(\operatorname{asChP}\left(\operatorname{gave}\left(f_{\text {seq }}^{1}\left(X_{\mu}\right)\right)(\mathbf{b})\right)\right)(\mathbf{t}) \wedge \operatorname{onSat}\left(\operatorname{asNYG}\left(\operatorname{gave}\left(f_{\text {seq }}^{2}\left(X_{\mu}\right)\right)(\mathbf{s})\right)\right)(\mathbf{t})
\end{aligned}
$$

We also obtain intuitively correct truth conditions for sentences such as the following, where two symmetrical terms exhibit interdependency with each other without being mediated by a separate plural term (unlike (73b) above):

a. Different students bought different books.

b. The same student bought different books.

The derivation proceeds by abstracting over the subject and object positions and 'respectivizing' the relation thus obtained, and then 'quantifying-in' the symmetrical terms in the subject and object positions one by one. This yields the following translation for (78a):

$$
\begin{aligned}
& \exists X_{\mu} . \forall i \in X_{\mu} \cdot \operatorname{student}(i) \wedge \forall i, j \in X_{\mu}\left[f_{\text {seq }}^{i} \neq f_{\text {seq }}^{j} \rightarrow i \neq j\right] \wedge \\
& \quad \exists Y_{\mu} . \forall i \in Y_{\mu} \cdot \operatorname{book}(i) \wedge \forall i, j \in Y_{\mu}\left[f_{\text {seq }}^{i} \neq f_{\text {seq }}^{j} \rightarrow i \neq j\right] \wedge \operatorname{resp}(\text { bought })\left(X_{\mu}\right)\left(Y_{\mu}\right)
\end{aligned}
$$

This asserts of an existence of a set of students and a set of books such that the buying relation is a bijection between these two sets. Thus, no two students bought the same book and no two books were bought by the same students. This corresponds to one of the intuitively available readings of the sentence. In Sect. 4.2 (p. 45), we discuss a more complex type of reading for the same sentence according to which the sets of books that each student bought are different from one another, where, for any given pair of students $s_{1}$ and $s_{2}$, there could be a partial (but not total) overlap between the sets of books that $s_{1}$ and $s_{2}$ respectively bought.

Examples like the following (80) in which the universal quantifiers every and each interact with symmetrical predicates can be analyzed by treating NPs containing universal quantifiers like every/each $N$ as maximal pluralities satisfying the description $N$ that are obligatorily associated with a distributive or 'respective' operator (see also Barker (2007) for a similar idea, but one that is technically implemented in a somewhat different way). ${ }^{20}$

$$
\{\text { Every/Each\} student read }\{\text { the same book/a different book\}. }
$$

The assumption that universal quantifiers in English (effectively) denote 'sums' (or pluralities) is advocated by several authors, including Szabolcsi (1997), Landman (2000), Matthewson (2001) and Champollion (2010). ${ }^{21}$ This assumption also accounts for the interactions between

\footnotetext{
${ }^{20}$ As noted at the end of Sect. 3.2.1, we take distributive readings to be derived by the resp operator we posit in our system, following a proposal by Bekki (2006). Our assumption here is in the same spirit as G\&K's suggestion of unifying the distributive and 'respective' operators in their system.

${ }^{21}$ Dotlačil (2010) questions this assumption by noting the infelicity of the following (the judgments are his):

(i) a. *Each boy each read a book.

b. *Each boy read a book each.

c. *Each boy talked to each other.
} 
universal quantifiers and 'respective' and summative predicates as exemplified by (21) from Sect. 2. In the present setup, the obligatory 'distributive' nature of every and each can be captured by specifying in their lexical entries that they take as arguments sentences missing multiset-denoting NPs, just like symmetrical terms. This can be done by syntactically encoding the semantic distinction between multiset-denoting and non-multiset-denoting NPs via some feature. ${ }^{22}$

The present analysis straightforwardly extends to scope interactions between symmetrical predicates and negation and quantifiers in examples like the following:

a. John and Bill didn't read the same book.

b. John and Bill didn't read different books.

c. Every boy gave every girl a different poem.

(81a) has a reading (perhaps the most prominent one) which seems to intuitively mean the same thing as 'John and Bill read different books'. Similarly, (81b), again on its perhaps most prominent reading, seems to mean essentially the same thing as 'John and Bill read the same book(s)'. By having the negation scope over the symmetrical predicate (which is straightforward by adopting the analysis of auxiliaries proposed in Kubota and Levine (2014a)), we obtain the following translations for (81a) and (81b):

$$
\begin{array}{ll}
\text { a. } & \neg\left[\exists X_{\mu} \forall i \in X_{\mu} \cdot \operatorname{book}(i) \wedge \forall i, j \in X_{\mu}[i=j] \wedge \operatorname{read}\left(f_{\text {seq }}^{1}\left(X_{\mu}\right)\right)(\mathbf{j}) \wedge \operatorname{read}\left(f_{\text {seq }}^{2}\left(X_{\mu}\right)\right)(\mathbf{b})\right] \\
\text { b. } & \neg\left[\exists X_{\mu} \cdot \forall i \in X_{\mu} \cdot \operatorname{book}(i) \wedge \forall i, j \in X_{\mu}\left[f_{\text {seq }}^{i} \neq f_{\text {seq }}^{j} \rightarrow i \neq j\right] \wedge \operatorname{read}\left(f_{\text {seq }}^{1}\left(X_{\mu}\right)\right)(\mathbf{j}) \wedge\right. \\
& \left.\operatorname{read}\left(f_{\text {seq }}^{2}\left(X_{\mu}\right)\right)(\mathbf{b})\right]
\end{array}
$$

It may appear that these truth conditions do not quite match the intuitive meanings of the sentences. Both (82a) and (82b) are true in a situation in which neither John nor Bill read any book. But intuitively, both (81a) and (81b) seem to mean that both John and Bill read at least one book. We do not attempt here to characterize the nature of this implication, but given that it is available in the non-negative counterparts of (81a) and (81b), and moreover seems to survive other presupposition holes (such as conditionals), it is most likely a presupposition of same/different. By taking this implication into account, the intuitively observed meanings of $(81 \mathrm{a})$ and $(81 \mathrm{~b})$ do in fact follow from $(82 \mathrm{a})$ and $(82 \mathrm{~b}) .^{23}$

As noted by Bumford and Barker (2013), (81c) is ambiguous between two readings: if the subject quantifier scopes over the object quantifier, it means that no boy gave the same

But the awkwardness of these examples seems to be due to the redundant marking of distributivity by the same word (albeit perhaps in distinct uses of it; compare, for example, the sentences in (i) with ?? All boys all went swimming, which is degraded for precisely the same reason of redundancy). Thus, we do not take these data to provide a convincing counterevidence to the family of 'universal as sum' type approach.

${ }^{22}$ One issue that remains is why the NP containing different is singular if the licensor is a distributive quantifier rather than a plural NP. In order to get our compositional mechanism yield the right result, we need to assume that a different book in (80) denotes a multiset just like different books. We suspect that the singular marking here is a matter of morphological agreement with the licensor, but leave a detailed investigation of this matter for a future study.

${ }^{23}$ Note, however, that this by no means means that (81a) and (81b) are truth conditionally equivalent to the non-negative versions of each other. Rather, the relation between them is that the truth conditions of these sentences, when augmented with typical (but cancellable) implicatures they are associated with (that is, the maximality implicature that the books in question are the only ones that John and Bill respectively read), effectively amount to the same as the truth conditions of the non-negative versions of each other plus their implicatures. 
poem to multiple girls, whereas if the object quantifier scopes over the subject quantifier, the sentence means that no girl received the same poem from multiple boys. Since our approach has a fully general mechanism for scope-taking for quantifiers and symmetrical predicates (via hypothetical reasoning involving $\uparrow$ ), this scope ambiguity is straightforwardly predicted.

We now turn to an analysis of summative predicates such as a total of $\$ 10,000$. The approach to symmetrical predicates above uses the resp operator to create pairings between 'corresponding' elements of two multisets, and then imposes a further condition on one of the two multisets involved. The (in)equality relation incorporated in this analysis is only one possible condition that could be so imposed, however; theoretically there are an unlimited number of other possible conditions, and we could expect a certain variety in the way natural language grammars exploit such possibilities. It turns out that we indeed see evidence of exactly this type of variety (for example, an average of $X$ is yet another such expression, as discussed by Kennedy and Stanley (2008)). In particular, in examples like (83) involving summative predicates, the multiset elements are required to (together) satisfy a quantity condition: taking $X_{\mu}$ and $Y_{\mu}$ to be the multisets consisting respectively of books that John bought and Bill received, then, roughly speaking, (83) asserts that the number of books contained in the union of $X_{\mu}$ and $Y_{\mu}$ is $100 .{ }^{24}$

John bought and Bill received a total of 100 books last year.

In other words, the condition 'adds up to 100' is imposed on the members of the multiset, instead of the (in)equality or similarity relations.

To capture this idea, we once again treat the relevant expressions as GQs over multisetdenoting terms of type $\mathrm{S} \uparrow(\mathrm{S} \uparrow \mathrm{NP})$, assigning to a total of the following meaning:

$$
\begin{aligned}
& \lambda \varphi_{1} \lambda \varphi_{2} \lambda \sigma . \sigma\left(\text { a o total } \circ \text { of } \circ \varphi_{1} \circ \varphi_{2}\right) ; \\
& \lambda n \lambda P \lambda Q . \exists X_{\mu} \cdot \#\left(X_{\mu}\right)=n \wedge P\left(X_{\mu}\right) \wedge Q\left(X_{\mu}\right) ; \mathrm{S} \uparrow(\mathrm{S}\lceil\mathrm{NP})\lceil\mathrm{N}\lceil\mathrm{Num}
\end{aligned}
$$

This operator takes some number $n$, a property denoted by a common noun $P$, and a predicate of multisets $Q$ and asserts the existence of a multiset $X_{\mu}$ that contains $n$ entities that satisfy the property $P$ (we assume that the counting function \# counts the number of atomic entities contained in the multiset, which does not necessarily coincide with the cardinality of the multiset $\left.\left|X_{\mu}\right|\right)$ and where $X_{\mu}$ itself satisfies the property $Q$. Since $Q$ is a predicate of multisetdenoting terms, this effectively means that $X_{\mu}$ enters into a 'respective' relation with some other multiset-denoting term in the sentence. The multiset $X_{\mu}$ can be thought of as a possible partitioning of some plural entity into subportions that can respectively be related to the other multiset(s), which, in the case of (83), is contributed by the other plural NP.

(83) is then analyzed in a way parallel to the symmetrical predicate example above. We first derive a sentence in which a hypothetically assumed multiset-denoting expression $\left(\varphi_{1}\right.$; $X_{\mu} ; \mathrm{NP}$ ) enters into a 'respective' predication with an overt conjoined term, which, in this case, is a 'nonconstituent' John bought and Bill received (note that the translation we give in (85) is simplified taking into consideration $f_{\text {seq }}=f_{\text {say }}$; the full derivation is given in (123)):

$$
\text { john } \circ \text { bought } \circ \text { and } \circ \text { bill } \circ \text { received } \circ \varphi_{1} ; \operatorname{bought}\left(\mathbf{j}, f_{\text {seq }}^{1}\left(X_{\mu}\right)\right) \wedge \operatorname{received}\left(\mathbf{b}, f_{\text {seq }}^{2}\left(X_{\mu}\right)\right) ; \mathrm{S}
$$

\footnotetext{
${ }^{24}$ For the sake of exposition, we give here an analysis of an example in which the argument of a total of is countable rather than mass. Extending the analysis to mass terms is straightforward (it merely involves replacing the counting function \# in (84) with some suitable measure function), once we introduce sums as the domain for the denotations of uncountable objects.
} 
By abstracting over $X_{\mu}$, we obtain an expression of type $\mathrm{S} \backslash \mathrm{NP}$, which is given as an argument to a total of 100 books which denotes a GQ over multisets, syntactically of type $\mathrm{S} \uparrow(\mathrm{S}\lceil\mathrm{NP})$. This yields the final translation in (86), which captures the intuitively correct meaning of the sentence:

$$
\begin{aligned}
& \operatorname{total}(\mathbf{1 0 0})(\text { book })\left(\lambda X_{\mu} \cdot \operatorname{bought}\left(\mathbf{j}, f_{\text {seq }}^{1}\left(X_{\mu}\right)\right) \wedge \operatorname{received}\left(\mathbf{b}, f_{\text {seq }}^{2}\left(X_{\mu}\right)\right)\right) \\
& =\exists X_{\mu} . \#\left(X_{\mu}\right)=\mathbf{1 0 0} \wedge \operatorname{book}\left(X_{\mu}\right) \wedge \operatorname{bought}\left(\mathbf{j}, f_{\text {seq }}^{1}\left(X_{\mu_{2}}\right)\right) \wedge \operatorname{received}\left(\mathbf{b}, f_{\text {seq }}^{2}\left(X_{\mu_{2}}\right)\right)
\end{aligned}
$$

\section{Comparisons and larger issues}

There are some issues worth commenting on at this point, in relation to closely related approaches from the literature that we draw on (Sect. 4.1), the larger literature on plurality (Sect. 4.2), and (even more generally) certain computational concerns regarding the use of empty operators (Sect. 4.3). We attend to these issues in this section.

\subsection{Comparisons with related approaches}

In the previous section, we have proposed a unified analysis of 'respective', symmetrical and summative predicates. The key components of our analysis are the treatment of expressions involving conjunction as denoting multisets and the flexible syntax-semantics interface of Hybrid TLCG. As demonstrated above, in our analysis, hypothetical reasoning for relating the relevant multisets in the 'respective' manner that underlies the semantics of these expressions interacts fully systematically with hypothetical reasoning for forming syntactic constituents that enter into that 'respective' predication. While our proposal builds on several key insights from previous proposals on these phenomena, we are unaware of any other proposal, at any level of formal explicitness, which accounts for the same range of data for which we have provided an explicit account. In this section, we discuss three previous approaches, namely, Gawron and Kehler (2004), Barker (2007, 2012) and Chaves (2012), that are closely related to our own, and discuss their key insights as well as limitations.

We focus on these three approaches here since, as we discuss below, we build on and combine key ideas that are most explicitly embodied in these proposals. But before moving on, we would like to briefly comment on the relations to other previous proposals. As for symmetrical predicates in particular, there are various accounts that contain important insights but which are not explicitly formalized, such as Dowty (1985), Carlson (1987) and Oehrle (1996). See Barker (2007) for a useful summary of these previous proposals. Barker's proposal and our own refinement of it can be thought of as an attempt to explicitly formalize the analytic intuitions embodied in these earlier works.

A more formally developed analysis of symmetrical predicates has recently been offered by Brasoveanu (2011). Though technically implemented in a different way, Brasoveanu's (2011) analysis of different embodies essentially the same analytic intuition as ours (see also Dotlačil (2010) and Bumford and Barker (2013)). Brasoveanu accounts for the pairwise matching between the sets of objects designated by the NP containing different and the correlate NP (i.e. a quantifier or a plural) via a device in an extended DRT called 'plural information states', which are formally sets of assignment functions and which can be thought of as stack-like objects. There is an obvious relation between multisets (with sequence functions) 
and stacks in that they are both formal constructs that keep track of the internal structures of complex objects with some ordering imposed on its elements. ${ }^{25}$

Moltmann (1992) perhaps deserves a special comment as well. In this work, Moltmann develops an approach to coordination which is essentially a version of multidominance analysis. This allows her to analyze interactions between RNR and symmetrical predicates such as the following via the notion of 'implicit coordination', where John and Mary, 'parallel' elements within conjuncts in RNR, are effectively treated as if they were coordinated for the purpose of interpretation of the shared element the same book.

John read, and Mary reviewed, the same book.

This approach raises many questions about compositionality and the architecture of the syntax-semantics interface (in particular, it is unclear what exactly is the status of the 'implicitly coordinated' John and Mary within the overall interpretation of the sentence). Moreover, since Moltmann's (1992) approach and ours start from totally different sets of assumptions, comparing the two directly is perhaps not very useful. However, there are two points which seem to be worth noting. First, although the key underlying intuitions are similar, Moltmann takes the syntactic coordinate structure to provide the basis for 'respective' readings (and related phenomena). Following authors such as Pullum and Gazdar (1982) and Gawron and Kehler (2004), we take this assumption to be implausible, since this account does not extend in any straightforward way to 'respective' readings of non-conjoined plural NPs. The other point which seems worth noting is that, unlike RNR, DCC seems less straightforward to analyze in a multidominance-type approach. The difficulty essentially lies in the fact that, unlike RNR, the shared string in DCC involves two separate constituents. It is unclear how the semantics of the sentence can be properly computed in such a complex multidominance structure (especially in examples involving interactions with 'respective', symmetrical and summative predicates). Moltmann does not discuss how her approach may be extended to DCC, and, so far as we are aware, this issue has not been addressed in any of the more recent variants of multidominance analyses of coordination such as Bachrach and Katzir (2007, 2008).

Finally, we would like to briefly comment on event-based approaches such as Lasersohn (1992). One might think that in such approaches, by introducing the notion of 'event sums', many of the examples discussed above can be treated without modifying the semantics of conjunction radically. In fact, this is precisely the point that Lasersohn (1992) argues for in his analysis of the adverb alternately (in examples such as The water was alternately hot and cold), which presents a similar problem as 'respective' readings and related phenomena we have examined above. However, Lasersohn's analysis deals only with the very basic cases of conjunction at the S (or VP) level-or, more precisely, cases in which the summing and teasing apart of events will matter only at the S level-and even for such examples, the extension to event-based semantics that he introduces (such as the notions of 'simple' and 'uniform' events) is nontrivially complex (moreover it is unclear whether there is any independent motivation for these elaborate abstract notions). Given this, though we do not

\footnotetext{
${ }^{25}$ Since Brasoveanu's approach and our own primarily focus on different sets of issues pertaining to the semantics of symmetrical predicates, a direct comparison does not seem to be very meaningful, but we would nevertheless like to note that it is unclear whether Brasoveanu's DRT-based system extends in any straightforward way to the interactions of symmetrical predicates (and related phenomena) and NCC.
} 
have a concrete proof of failure (and we do not attempt to construct one here), we feel justified to say that, at the very least, an attempt to extend an event-based approach to 'respective' predication involving various types of conjunction at a subsentential level, especially those involving NCC, does not seem to be straightforward. ${ }^{26}$

\subsubsection{Gawron and Kehler (2004)}

As noted in the previous section, our own analysis builds directly on Gawron and Kehler's (2004) (G\&K's) proposal in the core semantic analysis of 'respective' readings. The key difference between the two is that G\&K use sums to model the meaning of conjunction whereas we use multisets for the same purpose. As we discuss below, this multiset/sum difference in the two approaches has some important implications when extending the analysis to symmetrical predicates. Another, perhaps less essential (but nonetheless important) difference between the two approaches is that G\&K's proposal is formulated in a strictly phrase structure-based syntax-semantics interface. For this reason, their analysis, at least in its original form, does not extend straightforwardly to interactions with NCC. ${ }^{27}$

G\&K's approach presupposes that the argument of the sequencing function is a sum and not an atom. This assumption is necessary in their analysis for ensuring that the right matching of elements is established between the two sums in the case of 'respective' readings, but it causes a problem for at least a subset of symmetrical predicates. Recall from the previous section that the symmetrical predicate the same imposes an equality relation among each member of the multiset. If we recast this analysis in a sum-based analysis a la $\mathrm{G} \& \mathrm{~K}$, then the multiset (with some cardinality $n$ ) denoted by the same $N$ that is to be related to another multiset (with the same cardinality) in a pairwise manner collapses to a single

\footnotetext{
${ }^{26}$ Another potential issue with event-based approaches is that 'respective' predication and alternately sentences are found with non-eventive predicates as well (see also Barker $(2007,418$ ) for essentially the same point in connection to a similar example with same):

(i) a. The numbers 9 and 6 are odd and even, respectively.

b. The coefficients in this expansion of the function are alternately positive and negative integers.
}

It is of course conceivable to extend the notion of event to non-dynamic ones (and it is indeed tempting to think that in an example like (ib), a metaphorical extension of the notion of 'path' from a temporal/spatial domain to an atemporal/aspatial domain is involved), but even with such an extension, it is still considerably unclear how the proper 'events' can be individuated and distinguished from each other to derive the correct truth conditions for sentences such as those in (i).

${ }^{27}$ There is an additional technical problem in G\&K's proposal. Their analysis, in which 'respective' readings are analyzed via a series of successive applications of the distributive and 'respective' operators, turns out to be rather unwieldy in cases such as the following ((ib) is the same example as (53) above):

(i) a. John and Bill read and reviewed the book, respectively.

b. John and Bill sent the bomb and the letter to the president yesterday, respectively.

The problem essentially is that the distributive operator that G\&K posit (which is identical in all relevant respects to the distributive operator standardly assumed in the formal semantics literature) can only distribute a functor over the components of a sum of argument objects, not vice versa. But the analysis of (ia) requires distributing the object argument to the conjoined functor read and reviewed. See Kubota and Levine (2014d) for a more detailed discussion of this problem and possible solutions for it (the most straightforward of which is to extend the phrase structure-based setup of G\&K to an architecture like our own which recognizes hypothetical reasoning fully generally). 
object, since a sum of multiple 'tokens' of the same object collapses to that object itself (i.e. $a \oplus a=a$ by definition). But then, it would be predicted that John and Bill read the same book is infelicitous since in G\&K's analysis, the sequencing function is defined only for sums that have proper subparts (lifting this condition in their approach would incorrectly admit examples like \#Sue and Bob like Fred, respectively in their analysis). ${ }^{28}$

One might think that this problem could be circumvented by treating same in a different way (along the lines that, in the case of same, the mapping is not between two sums but rather between a sum and a single fixed object), but an essentially analogous problem arises with similar in a way that precludes any easy reformulation of the analysis. To see this, note first that the similarity condition that similar imposes on its multiset elements does not exclude a possibility that the elements are completely identical. Suppose, for example, Alice suggests to her collaborator Betty:

(88) Ok, Betty, let's work on these problems separately first, and then if we run into similar problems, let's get together and discuss.

They later confer by email and discover that they are stuck in exactly the same problem. Alice refuses to get together and discuss and insists that they keep working separately, since they've run into exactly the same problem, not similar problems. We think that Alice would be a perverse person in such a situation. Thus, the implication of 'similar but not the same' is arguably a Gricean implicature. But then, this means that if the multiset elements happen to be identical, a G\&K-based analysis predicts sentences containing similar to be infelicitous. In other words, (the if clause of) (88) is predicted to be infelicitous just in case Alice and Betty run into exactly the same problems. This does not seem to be a correct prediction.

It then seems fair to conclude that G\&K's sum-based approach does not extend straightforwardly to the analysis of symmetrical predicates. As we have discussed in Sect. 2, we take it that the parallel between 'respective' readings on the one hand and symmetrical and summative predicates on the other to be robust. Thus, in the absence of an explicit and fully general analysis of symmetrical predicates in a sum-based approach (in this connection, see also the discussion of the empirical problems of Barker's $(2007,2012)$ approach in the next section), we take our multiset-based analysis to be an improvement over G\&K's original sum-based analysis of 'respective' readings.

\subsubsection{Barker $(2007,2012)$}

Barker (2007) proposes an analysis of symmetrical predicates via the notion of 'parasitic scope', which captures the syntax-semantics interface underlying the compositional semantics of these predicates quite elegantly. In his analysis, same receives the following translation:

\footnotetext{
${ }^{28}$ Gawron and Kehler $(2004,174)$ claim that this assumption explains the ill-formedness of the following:
}

(i) ??Sue and Bob like Fred and Fred, respectively.

Our analysis does not rule out (i) via the combinatoric mechanism for licensing 'respective' readings alone. But note that the awkwardness of (i) (whose truth conditional content could be expressed much more perspicuously by Sue and Bob like Fred) derives from the fact that the same expression with identical reference is conjoined, without any good reason. This is independently bad regardless of whether the 'respective' reading is involved (cf., e.g., ?? Fred and Fred smiled). 


$$
\lambda \mathscr{F} \lambda X \exists \mathfrak{f} \forall x<{ }_{a} X . \mathscr{F}(\mathfrak{f})(x)
$$

Here, $\mathscr{F}$ is of type $($ et $\rightarrow$ et $) \rightarrow$ et. That is, $\mathscr{F}$ denotes a relation between adjectives (i.e. modifiers of common nouns) on the one hand and individuals on the other, with $X$ a variable over sums of individuals and $\mathfrak{f}$ a variable over CHOICE FUNCTIONS (a choice function is a function that takes a set as argument and returns as output a singleton set containing a member of that set). Roughly speaking, same converts a relation between functors on some property on the one hand and an individual on the other into a relation between inhabitants of that property on the one hand and sums of individuals on the other, guaranteeing a unique inhabitant of that property to which each individual in the sum is mapped. Thus, for example, in the case of (90), an abstraction first on a variable over individual types and then over adjective types yields the relation $\lambda f \lambda y \cdot \operatorname{read}(\iota(f(\mathbf{b o o k}))(y))$ (where $\iota$ is the iota operator denoted by the definite article the).

(90) John and Bill read the same book,

The same operator in (89) then maps this relation to a relation between the sum of individuals $\mathbf{j} \oplus \mathbf{b}$ on the one hand and a single element of the set book such that each member of the sum is in the read relation to that element.

It should be clear from the above that our own analysis takes Barker's work as its basis. In particular, we take Barker's double abstraction treatment of same as the core of our own compositional analysis, though the specific semantic analyses differ in important ways. We aim at a unitary analysis of symmetrical, 'respective' and summative predicates; hence, the key semantic commonality we have identified in these three cases - the mapping relationship between elements of two (or more) different composite data structures - correspond to a single source, the resp operator, which cross-cuts the specific semantic (and pragmatic) properties of the three. By contrast, in Barker's analysis, the operation corresponding to our resp is directly encoded in the lexical meaning of symmetrical predicates.

But quite apart from this difference, the lack of a recursive mechanism that keeps track of the structure of a sum/multiset-type object entails severe empirical difficulties when multiple instances of symmetrical predicates are present. We illustrate this problem with (91), for which Barker's analysis yields a particularly strange semantics. ${ }^{29}$

(91) John and Bill gave different things to different people.

Barker (2007) gives the semantics of different as in (92):

$$
\lambda \mathscr{F} \lambda X \forall \mathfrak{g} \forall z, v<_{a} X .[\mathscr{F}(\mathfrak{g})(z) \wedge \mathscr{F}(\mathfrak{g})(v)] \rightarrow[z=v]
$$

with which the translation in (93b) is assigned to (93a) $(\epsilon$ here is the meaning of the indefinite article $a$; since the choice function returns a singleton set, the choice of the article (between the for same and a for different) is immaterial in Barker's analysis).

a. John and Bill read a different book.

\footnotetext{
${ }^{29}$ Barker (2012) partially addresses the multiple symmetrical predicate issue by revising the translation for same in Barker (2007) slightly, removing the distributive operator from the meaning of same (and instead assuming that it is implicit in the lexical meaning of the verb). However, this approach does not seem to work for the case of different (within the set of assumptions that Barker (2007, 2012) makes), and Barker (2012) remains silent about cases like (91).
} 


\section{b. $\forall \mathfrak{f} \forall z, v<_{a} \mathbf{j} \oplus \mathbf{b}[\operatorname{read}(\iota(\mathfrak{f}($ book $)))(z) \wedge \operatorname{read}(\epsilon(\mathfrak{f}($ book $)))(v)] \rightarrow[z=v]$}

To paraphrase, (93b) says that whatever choice function one chooses, the only way in which two (potentially distinct) people out of the set $\{\mathbf{j}, \mathbf{b}\}$ read the book that the choice function returns is when the two people are the same ones. In other words, there is no single common book that John and Bill both read.

Assuming Barker's semantics for different, and following the procedure for multiple same discussed in Barker (2012), we wind up with the translation for the VP for (91) in (94).

$$
\begin{aligned}
\lambda U \forall w, y<{ }_{a} U \forall \mathfrak{f} \forall \mathfrak{g} \lambda W\left[\forall z, v<<_{a} W[\operatorname{gave}(\iota(\mathfrak{f}(\text { thing })))(\epsilon(\mathfrak{g}(\text { person })))(z) \wedge\right. \\
\text { gave }(\iota(\mathfrak{f}(\text { thing })))(\epsilon(\mathfrak{g}(\text { person })))(v)] \rightarrow[z=v]](w) \\
\wedge \lambda W\left[\forall z, v<<_{a} W[\text { gave }(\iota(\mathfrak{f}(\text { thing })))(\epsilon(\mathfrak{g}(\text { person })))(z) \wedge\right. \\
\quad \text { gave }(\iota(\mathfrak{f}(\text { thing })))(\epsilon(\mathfrak{g}(\text { person })))(v)] \rightarrow[z=v]](y) \rightarrow[y=w]
\end{aligned}
$$

We see here a subtyping mismatch problem whose resolution leads to a severe mischaracterization of the truth conditions of the sentence. The problem in a nutshell is that each token of different introduces an abstraction on a sum type, each of whose atoms are to be related to a member of some set of entities which is identical to no other member of that set. But only the wider-scoping token of different will get an actual sum (in this case, john $\oplus$ bill) supplied as its argument; the narrower-scoping token will be able to apply only to the universally bound atomic elements introduced by the wider-scoping instance of different. The only way we can see to resolve this apparent incoherence in the semantics of such examples is to treat the 'part-whole' relation $<_{a}$ as simple equality in the case where the second relatum is an atom, as indeed intimated in Barker (2012) in his treatment of the multiple same examples. But the assumption that $x<_{a} u$ entails $u=x$ has, as a corollary, the consequence that $x, y<{ }_{a} u$ entails that $u=x=y$. The result is that (94) reduces to (95):

$$
\begin{aligned}
\lambda U \forall w, y<{ }_{a} U \forall \mathfrak{f} \forall \mathfrak{g}[[\operatorname{gave}(\iota(\mathfrak{f}(\text { thing })))(\epsilon(\mathfrak{g}(\text { person })))(w)] & \rightarrow[w=w] \wedge \\
{[\operatorname{gave}(\iota(\mathfrak{f}(\text { thing })))(\epsilon(\mathfrak{g}(\text { person })))(y)] } & \rightarrow[y=y]] \rightarrow[y=w]
\end{aligned}
$$

But this makes no sense. What we have in (95) is an implication, whose antecedent is a tautology (itself composed of a conjunction of tautologies of the same general form $\psi \rightarrow(\alpha=$ $\alpha)$ ), which means that the whole conditional statement is equivalent to its consequent $y=w$. The variables $w$ and $y$ range over the atoms of the sum that (95) takes as its argument. Thus, it is predicted that (91) means that John and Bill are the same person. ${ }^{30}$

In summary, Barker's analysis of symmetrical predicates loses generality in two directions, and these problems, we believe, essentially derive from the same limitation in his analysis. On the one hand, unlike G\&K's and our proposal, Barker's analysis lacks a mechanism for making the internal structure of a sum simultaneously visible to multiple tokens of same/different. For this reason, it does not extend to multiple same/different sentences fully generally. On the other hand, the fundamentally parallel semantic action of 'respective' interpretations and

\footnotetext{
${ }^{30}$ Similar difficulties arise in an only slightly less striking fashion in John and Bill put the same object in different boxes, where Barker's analysis predicts that on the inverse scoping of the two symmetrical predicates it is impossible for John to have put any object in a box that Bill put some other, distinct object in. While the surface scope (same $>$ different) does yield the correct interpretation for this example, the picture changes when the subject is singular rather than plural: in the case of John put the same object in different boxes, the surface scoping yields a tautology predicting that the sentence is true in all conceivable circumstances.
} 
summative predicates cannot be captured in Barker's implementation because the crucial sum-to-individual mapping is directly encoded in the lexical meaning of the symmetrical predicate operators in his proposal. To remove these obstacles, a different strategy seems to be needed, in which a mapping between composite objects is mediated by a separate general operator that allows for recursive application, as in G\&K's and our own proposal. ${ }^{31}$

\subsubsection{Chaves (2012)}

Chaves' (2012) account identifies 'respective', symmetrical and summative interpretations as unitary phenomena (see especially pp. 319-321) - a view we inherit in our own analysis. The mechanism Chaves proposes for the compositional analysis of these predicates is however fundamentally different from our approach; Chaves takes 'respective' readings in cases such as Bill and Tom invited Sue and Anne to the party (respectively) to be nothing more than particular instances of the so-called cumulative reading (Scha 1981), along the lines discussed in Sect. 2 above (p. 4).

But as noted by Gawron and Kehler (2004) in their critique of Schwarzschild's (1996) analysis of 'respective' readings, such an approach does not extend to examples like (96) (= (1)), in which one of the sums related in the 'respective' manner is a sum of predicates rather than a sum of entities:

John and Bill sang and danced, respectively.

$$
\text { (= 'John sang and Bill danced') }
$$

Chaves therefore proposes the translation in (97) for the conjunction word and, which, according to him, has the effect of providing two possible interpretations for (96) and similar examples (we have modified Chaves's (2012) original translation slightly to accommodate it with the description of the and operator he gives in the text - note that the event variable $e$ isn't existentially bound in our reformulation; nothing crucially hinges on this modification).

$$
\begin{aligned}
& \lambda P \lambda Q \lambda z_{0} \ldots \lambda z_{n} \lambda e \cdot\left[e=\left(e_{1} \oplus e_{2}\right) \wedge Q\left(x_{0}\right) \ldots\left(x_{n}\right)\left(e_{1}\right) \wedge P\left(y_{0}\right) \ldots\left(y_{n}\right)\left(e_{2}\right) \wedge z_{0}=\left(x_{0} \oplus\right.\right. \\
& \left.\left.y_{0}\right) \wedge \ldots \wedge z_{n}=\left(x_{n} \oplus y_{n}\right)\right]
\end{aligned}
$$

This operator conjoins two relations, and either identifies (i.e., if $x_{n}=y_{n}$ ) or distributes (i.e., if $x_{n} \neq y_{n}$ ) their conjoined shared dependents. With (97), (96) receives the following interpretation (after the event variable $e$ is existentially closed):

$$
\exists e^{\prime \prime} . e^{\prime \prime}=\left(e_{1} \oplus e_{2}\right) \wedge \operatorname{sang}\left(x_{0}\right)\left(e_{1}\right) \wedge \operatorname{danced}\left(y_{0}\right)\left(e_{2}\right) \wedge(\mathbf{j} \oplus \mathbf{b})=\left(x_{0} \oplus y_{0}\right)
$$

The idea is that if $x_{n}=y_{n}$, then both John and Bill sang and both danced, whereas if $x_{n} \neq y_{n}$, then either John danced and Bill sang or Bill danced and John sang. The adverb respectively imposes a further constraint on the interpretation obtained above, to force the pairings $\mathbf{j}=x_{0}, \mathbf{b}=y_{0}$ (which effectively restricts the interpretation to the $x_{n} \neq y_{n}$ case).

Note that, in this analysis, except for complicating the meaning of and, no special mechanism is needed in the grammar to license 'respective' readings (and related phenomena),

\footnotetext{
${ }^{31}$ Given the parallel semantic action of symmetrical predicates on the one hand and 'respective' readings on the other, it is worth observing that Kubota's (2015) extension of Barker's (2007) analysis of same to 'respective' readings fails to generalize to multiple 'respective' readings. The problem in these cases is the same type mismatch dilemma blocking recursive application of operators: after the first application of the 'respective' operator, the result is a boolean conjunction whose parts are no longer accessible as sum components, making further application of the same operator in principle impossible.
} 
giving us an overall very simple analysis of a complex class of phenomena. But the success of this approach is only apparent; once we extend the data pool beyond the most simple class of examples (such as (96)), Chaves' analysis quickly becomes problematic. NCC/'respectively' interaction examples such as (99) illustrate the point clearly:

(99) I bet $\$ 50$ and $\$ 100$ with John on the football game and (with) Mary on the basketball game (respectively).

Since (99), by virtue of its nonconstituent conjuncts, cannot be directly interpreted, the syntactic source of this sentence must be presumed to arise under the prosodic ellipsis analysis Chaves explicitly assumes (as per Beavers and Sag (2004); Chaves (2008); Hofmeister (2010)), with (100) the necessary input to the semantic interpretation: ${ }^{32}$

(100) I bet $\$ 50$ and $\$ 100$ with John on the football game and (I) bet $\$ 50$ and $\$ 100$ (with) Mary on the basketball game (respectively).

No cumulative interpretations are available for the conjoined clauses or verb phrases in (100). Thus, the source of the 'respective' reading in (99) must arise from the action of the and operator in (97). But the necessary conditions on and are not satisfied in (100). In order for Chaves' setup to work as required, it is crucial that the arguments corresponding to $x_{k}, y_{k}$ be actual conjuncts, but this critical condition is not fulfilled in (100), where the terms that need to enter into the 'respective' relation belong to completely different clauses/VPs, appearing (on the surface string) to be parts of conjoined expressions only via the strictly prosodic deletion operation which yield the surface string (99). The operator in (97) therefore provides no account of how the 'respective' readings arise in examples of this type.

In a sense, Chaves' proposal can be thought of as an attempt to lexicalize (in the meanings of conjoined predicates) the effects of the 'respective' operator of the sort posited in G\&K's and our approach. While a strictly lexical approach may be attractive if it can handle all the relevant data uniformly, the discussion above suggests that such an approach is not general enough.

\subsection{A note on the treatment of plurality}

One might wonder how the multiset-based analysis of 'respective' readings and related phenomena presented above extends (or does not extend) to other interpretations of plural and conjoined expressions, such as collective and cumulative readings. These phenomena are

\footnotetext{
${ }^{32}$ Chaves (2012) extensively relies on the ellipsis strategy even in cases like the following that do not involve nonconstituent coordination:

(i) Different newspapers are running conflicting reports. The Guardian and the Telegraph reported that Michael Phelps won the silver medal and the gold medal respectively.

(Chaves 2012, 316)

According to Chaves (2012), the 'respective' reading of (i) is obtained from the underlying structure in (ii):

(ii) The Guardian and the Telegraph reported [that Michael Phelps won the silver medal] and [that Miehrel Phelps won the gold medal] respectively.
} 
themselves quite complex, and each of them deserves a treatment on its own. Thus, addressing them fully is clearly beyond the scope of the present paper. But given that the phenomena treated above are clearly related to the more general empirical domain of plurality, some comments seem to be necessary, and we will try to explicate our position here.

In a comparison of our multiset-based approach with a sum-based alternative (which is more standard at least for the treatment of plurality), it is useful to distinguish three properties of different types of formal objects that are (or can be) used for modelling entities having complex internal structures, so as to avoid confusion about points of (non-)controversy. Multisets have more complex structures than sums or sets in that they allow for identical elements to appear twice (a property that we call iterability), and they can be nested (nestability). Multisets are also different from tuples (which have also been used by some previous authors for the analysis of 'respective' readings and related phenomena-see Bekki (2006); Winter (1995); Kubota and Levine (2014d)) in that they do not keep track of ordering of elements in the data structure itself. A comparison of different data structures with respect to these properties is as follows:

\begin{tabular}{lccc} 
& ordering & iterability & nestability \\
\hline tuple & yes & yes & yes \\
multiset & no & yes & yes \\
set & no & no & yes \\
sum & no & no & no \\
\hline
\end{tabular}

To state the conclusion first, the only property we crucially need for our unified analysis of the three phenomena is iterability, hence our choice of multisets. Note from the discussion in Sect. 4.1.1 that G\&K's analysis in terms of sums does not extend straightforwardly to symmetrical predicates since sums lack this property. We do not make use of the nestability property in any crucial way in the analysis we have presented above (but note the brief discussion of extending the present approach to the treatment of cumulativity below; this is one possible place where the nestability of multisets may perhaps be exploited profitably). In fact, as one of the reviewers has pointed out, nestability even potentially complicates the analysis of plurals in a non-trivial manner. Here, we have to say that this is an unfortunate consequence. Ideally, we would want some formal object with iterability but not nestability. But as the above table makes clear, there is no known formal object that has the right property, at least none that we are currently aware of. Thus, it seems that we have to live with the artificial complication introduced by the nestability property of multisets, that is, the so-called 'overrepresentation' problem in the plurality literature (discussed below). But it should be noted that eliminating the unnecessary distinctions can always be done uniquely and unambiguously. Given this, whether overrepresentation is merely a technical complication or it has empirical consequences as well seems to be a debatable issue. We do not aim to take a stance on this debate here, and simply acknowledge that the present proposal suffers from this complication, in the same way that group-based analyses of plurality does.

Regarding the overrepresentation issue, we find Schwarzschild's (1996) argument against groups (another type of formal object that has the nestability property) convincing. The argument runs roughly as follows. An example like (102) at first sight unambiguously means that the separation was done by kind, apparently motivating a group-based analysis of plurals. 
But things are not so clear-cut once we take into consideration more complex examples like (103).

(102) The cows and the pigs were separated.

a. The cows and the pigs were separated by age.

b. The animals were separated by age. That is, the cows and the pigs were separated.

In a world in which there are only cows and pigs, the second sentence of (103b) means the same thing as (103a). Schwarzschild (1996) concludes from this that the subgrouping of a sum in sentences like (102) is provided by a contextual parameter (which he calls 'covers'), rather than explicitly represented at the level of semantics (via groups, or whatever formal objects that have the property of nestability). It indeed seems that, if one limits one's attention to the treatment of plurality (in the nominal domain), the overrepresentation problem of the group-based approach (which our multiset-based approach inherits) speaks in favor of Schwarzschild's neo-Linkean sum-based approach.

There are, however, other points of consideration that come into play once we extend our empirical domain to a wider range of linguistic phenomena (outside of the distributive readings of nominal pluralities). First, as noted by G\&K, though Schwarzschild (1996) sketches an extension of his cover-based analysis to the NP-NP cases of 'respective' readings (e.g. (104a)), his analysis does not generalize fully to other types of 'respective' readings (e.g. (104b); see the discussion of Chaves' (2012) proposal above for a related point).

$$
\begin{aligned}
& \text { a. John and Bill married Mary and Sue, respectively. } \\
& \text { b. John and Bill walked and talked, respectively. }
\end{aligned}
$$

It is unclear whether an extension of Schwarzschild's (1996) cover-based analysis is motivated for examples like (104b), and more generally, for a still larger (and more complex) dataset encompassing examples involving interactions with NCC such as (4c,d).

Another, perhaps more important, point is that, as we have discussed in Sect. 4.1.1, a multiset-based analysis enables a straightforward extension to the analysis of symmetrical predicates whereas a sum-based analysis doesn't. To be fair, Schwarzschild's (1996) proposal was not intended to cover symmetrical predicates, but to the extent that one finds the parallel between 'respective' readings and symmetrical predicates (of the sort noted in the previous section) intriguing, a unified analysis seems more preferable.

For these reasons, we tentatively conclude that overrepresentation in the domain of nominal plurality is a price that has to be paid in view of the overall generality of the analysis in a wider empirical domain. We recognize that this is a potentially controversial claim, and would like to invite responses from those who subscribe to the neo-Linkean, sum-based treatment of plurality.

Regarding the 'ordering' issue (i.e. multisets vs. tuples), there are some reasons that have ultimately convinced us that a multiset-based analysis presented above is better than a tuplebased one (of the sort proposed by Bekki (2006); Winter (1995); Kubota and Levine (2014d)) that we entertained in an earlier version of the present paper. Note first that, as noted by Lasersohn (1988, 87-88), representing the meanings of conjoined NPs in the form of tuples complicates the semantics of collective readings in examples like the following: 
(105) Lennon and McCartney wrote Across the Universe and Sexy Sadie. (='They both co-authored')

The ordering of elements doesn't make any truth-conditional difference for collective readings, and to capture this fact on a tuple-based approach, one would need to introduce some extra assumptions.

Even more problematic for the tuple-based approach are examples like (106), where an order-insensitive bijective relation is established between two conjoined expressions:

a. The front and the back of the ship are called the bow and the stern, but which is which?

(Chaves 2012)

b. We know houses four and five are the Swede and the German, but which is which?

(Chaves 2012)

To account for these examples, a tuple-based analysis would have to assume an operator that reorders the elements of a tuple and underspecifies the ordering.

Finally, we would like to discuss briefly how one might go about extending the present multiset-based analysis of 'respective' readings to cumulative predication. With a slight extension, the present approach opens up a simple way of characterizing cumulative readings. Specifically, all we need to do is to assume that multisets corresponding to plural terms can contain elements that are themselves multisets of individuals rather than just atomic individuals. ${ }^{33}$ We sketch here a basic analysis of (107) and then discuss some implications.

(107) A total of 4 students read a total of 5 books.

Assuming that we have four students s1, s2, s3, s4 and five books b1, b2, b3, b4, b5 and that the reading relation that holds between the two sets is given by the following:

$$
\{\langle\mathbf{s 1}, \mathbf{b 1}\rangle,\langle\mathbf{s 1}, \mathbf{b 2}\rangle,\langle\mathbf{s 2}, \mathbf{b 1}\rangle,\langle\mathbf{s 2}, \mathbf{b 2}\rangle,\langle\mathbf{s 2}, \mathbf{b 3}\rangle,\langle\mathbf{s 3}, \mathbf{b 2}\rangle,\langle\mathbf{s 3}, \mathbf{b 4}\rangle,\langle\mathbf{s 4}, \mathbf{b 5}\rangle\}
$$

then, the situation can be modelled by a 'respective' predication between two multisets:

$$
\begin{aligned}
& X_{\mu}=\{\mathbf{s} 1, \mathbf{s} 2, \mathbf{s} 3, \mathbf{s} 4\} \\
& Y_{\mu}=\{\{\mathbf{b} 1, \mathbf{b} 2\},\{\mathbf{b} 1, \mathbf{b} 2, \mathbf{b} 3\},\{\mathbf{b} 2, \mathbf{b} 4\}, \mathbf{b} 5\}
\end{aligned}
$$

with the sequencing function $f_{\text {seq }}$ picking up elements from the two multisets in the order in which we have listed the elements in (109). Thus, the following translation that is assigned to the sentence compositionally by the present analysis suffices to capture the cumulative reading of (109):

$$
\exists X_{\mu} \cdot \#\left(X_{\mu}\right)=4 \wedge \operatorname{student}\left(X_{\mu}\right) \wedge \exists Y_{\mu} \cdot \#\left(Y_{\mu}\right)=5 \wedge \operatorname{book}\left(Y_{\mu}\right) \wedge \operatorname{resp}(\operatorname{read})\left(X_{\mu}\right)\left(Y_{\mu}\right)
$$

\footnotetext{
${ }^{33}$ Note that we model plural individuals in terms of multisets alone, eliminating sums altogether from the ontology of plurality. This still leaves room for sums as the possible denotations of mass objects. Since there is a functional mapping from multisets modelling plural entities in the present approach to the corresponding Linkean sums (it just involves eliminating nesting and duplication of identical elements), representing plurals by multisets does not mean that we lose the robust empirical generalization between nominal and verbal domains noted extensively in the mereological literature (Bach (1986); Krifka (1989, 1992), among many others).
} 
This extension offers a promising approach to characterizing the more complex reading for sentences involving two occurrences of different such as the following (cf. Sect. 3.2.2).

(111) Different students read different books.

The relevant reading links students to sets of books that (s)he read and asserts that for no pair of two students, the sets of books that they respectively read are completely identical. Thus, (111) is true on this reading in a situation (call it situation 1) described above in (108) but false in a situation where $\mathbf{s} \mathbf{1}$ read b3 in addition (call it situation 2), since in situation 2 , the sets of books that $\mathbf{s} \mathbf{1}$ and $\mathbf{s} \mathbf{2}$ read are exactly identical. By assuming that the multiset of students consists of atomic students but that the multiset of books can consist of sums of books, we can model this reading with our resp operator and the semantics for different already introduced above. The sentence comes out true in situation 1 but false in situation 2 , since, according to the semantics of different, each element of the book multiset needs to be distinct from each other, a condition satisfied in situation 1 but not in situation 2 .

The analysis of cumulativity sketched above, although preliminary, is promising in that it already extends straightforwardly to quite complex examples such as the following, a type of sentence originally discussed by Schein (1993), where cumulativity and distributivity interact:

(112) A total of three ATMs gave a total of 1000 customers two new passwords.

There is a reading of this sentence in which three ATMs is related to 1000 customers in the cumulative manner, and two new passwords distributes over each ATM-customer pair (thus involving 2000 distinct passwords issued).

To derive this reading, all we need to assume is that the plural term two new passwords denotes an ordinary cardinal quantifier that scopes below the 'respective' operator that establishes the cumulative relation between the other two plural terms (by alternating scope, we can account for other scope readings for the sentence too). The full derivation is given in (124) in Appendix A. The translation obtained is as follows:

$$
\begin{aligned}
& \operatorname{total}(\mathbf{3})(\operatorname{atm})\left(\lambda X _ { \mu } \cdot \operatorname { t o t a l } ( 1 \mathbf { k } ) ( \mathbf { c u s } ) \left(\lambda W_{\mu} .\right.\right. \\
& \left.\left.\bigwedge_{\mu} \operatorname{resp}(\lambda x \cdot \operatorname{dist}(\lambda y \cdot \operatorname{two}-\mathbf{p w}(\lambda z \cdot \operatorname{gave}(y)(z)(x))))\left(X_{\mu}\right)\left(W_{\mu}\right)\right)\right)
\end{aligned}
$$

Assuming that the three ATMs in question are atm1, atm2, and atm3, and that $f_{\text {seq }}$ picks up these ATMs in this order, the final translation reduces to the following:

$$
\begin{gathered}
\exists W_{\mu} \cdot\left|W_{\mu}\right|=3 \wedge \#\left(W_{\mu}\right)=1000 \wedge \text { customer }\left(W_{\mu}\right) \wedge \\
\operatorname{dist}(\lambda y \cdot \mathbf{t w o}-\mathbf{p w}(\lambda z \cdot \operatorname{gave}(y)(z)(\operatorname{atm} 1)))\left(f_{\text {seq }}^{1}\left(W_{\mu_{3}}\right)\right) \wedge \\
\operatorname{dist}(\lambda y \cdot \mathbf{t w o}-\mathbf{p w}(\lambda z \cdot \operatorname{gave}(y)(z)(\operatorname{atm} 2)))\left(f_{\text {seq }}^{2}\left(W_{\mu_{3}}\right)\right) \wedge \\
\operatorname{dist}(\lambda y \cdot \operatorname{two}-\mathbf{p w}(\lambda z \cdot \operatorname{gave}(y)(z)(\operatorname{atm} 3)))\left(f_{\text {seq }}^{3}\left(W_{\mu_{3}}\right)\right)
\end{gathered}
$$

This means that the total of 1000 customers can be partitioned into three groups such that for each of these groups, one of the three ATMs gave two distinct passwords to each individual in that group. This corresponds to the relevant reading of the sentence. 


\subsection{A note on empty operators}

One issue that the present proposal raises is the extensive use of empty operators. It is worth noting in this connection that a certain restricted form of Hybrid TLCG - specifically, one which eliminates empty operators and polymorphic specifications of lexical entries - has been shown to be decidable (Moot 2014), just like related approaches in TLCG such as Displacement Calculus and $\mathrm{NL}_{\lambda}$. Decidability is an important property when considering the formal computational properties of a grammatical framework, and this naturally raises the question of what to make of the empty operators and polymorphic specifications that we have extensively relied on in our analysis of 'respective' readings and related phenomena above.

Whether these operators can be eliminated without sacrificing the generality of the analysis proposed above too much is currently an open question. There are two points worth commenting on in this connection. First, empty operators introduced for the analysis of certain other syntactic phenomena (such as the ellipsis operators in Barker (2013) and Kubota and Levine (2014c)) are relatively 'harmless' in that they can be lexicalized in the meanings of words that have actual prosodic content (such as the wh remnant in Barker's (2013) analysis of sluicing and the auxiliary in Kubota and Levine's (2014c) analysis of pseudogapping and VP ellipsis). But it is not obvious whether the empty operators we have posited in the analysis of 'respective' readings and related phenomena above can be lexicalized similarly readily. Second, and relatedly, despite this concern, empty operators are already extensively employed in the plurality literature, for phenomena that are (at least in certain respects) less complex than those dealt with in the present paper. In fact, we do not know of any proposal that attempts to lexicalize the more 'basic', and much more widely discussed, standard distributive operator in the plurality literature. But given that human speakers have no trouble understanding sentences containing plurals, respectively, and symmetrical and summative predicates (at least simple ones), something more clearly needs to be said on this issue. Unfortunately, addressing this important and quite intriguing question properly is beyond the scope of the present paper, and we have to leave this task for future study.

\section{Conclusion}

Our analysis of 'respective' readings and related phenomena incorporates ideas from both G\&K's analysis of 'respective' readings and Barker's analysis of symmetrical predicates, and unifies these based on the key analytic idea due to Chaves (2012) that a single common mechanism is involved in the compositional semantic mechanism underlying these phenomena. While the strictly local approach in G\&K's original formulation and the nonlocal approach by Barker via 'parasitic scope' may initially look quite different, the effects of the two types of operations (or series of operations) that they respectively invoke are rather similar: they both establish some correspondence between the internal structures of two terms that do not necessarily appear adjacent to each other in the surface form of the sentence. The main difference is how this correspondence is established: G\&K opt for a series of local composition operations (somewhat reminiscent of the way long-distance dependencies are handled in lexicalist frameworks such as CCG and G/HPSG), whereas Barker does it by a single step of nonlocal mechanism (in a way analogous to a movement-based analysis of longdistance dependencies). Chaves's approach can be seen as an attempt to strictly lexicalize 
these effects, but as we have noted above, this approach is insufficiently general.

A question that arises at this point is whether we have gained any deeper understanding of the relationship between the respective solutions proposed by G\&K and Barker, by recasting them within the general syntax-semantics interface of Hybrid TLCG. We do think that we have. Note first that, by recasting these proposals in our setup, we have a clearer picture of the core mechanism underlying these phenomena. This in turn enabled us to overcome the major empirical limitations of both G\&K's analysis (with respect to NCC) and Barker's analysis (with respect to iterated symmetrical predicates).

But we can go even further. As discussed in detail in Kubota and Levine (2014d), the local and nonlocal modelling of 'respective' predication from the two previous works can be shown to have a very close relationship formally, since in Hybrid TLCG, the local composition rules for 'percolating up' the multiset structure from coordination that are crucially involved in G\&K's setup can be derived as theorems in a grammar that essentially implements Barker's approach of nonlocal 'respective' predication via hypothetical reasoning. More specifically, by introducing some auxiliary assumptions, the following two rules (where Rule 1 corresponds to G\&K's Dist operator and Rule 2 corresponds to the Dist' operator needed but apparently missing from G\&K's setup) are both derivable as theorems in the system we presented in Sect. 2 (we owe the key idea to Bekki (2006); for proofs, see Kubota and Levine (2014d)):

a. RULE 1

$$
\frac{a ; \mathcal{F} ; A / B \quad b ;\left\{\boldsymbol{a}_{\mathbf{1}} \ldots \boldsymbol{a}_{\boldsymbol{l}}\right\} ; B}{a \circ b ;\left\{\mathcal{F}\left(\boldsymbol{a}_{\mathbf{1}}\right) \ldots \mathcal{F}\left(\boldsymbol{a}_{\boldsymbol{l}}\right)\right\} ; A}
$$

b. RULE 2

$$
\frac{a ;\left\{\mathcal{F}_{\mathbf{1}} \ldots \mathcal{F}_{\boldsymbol{n}}\right\} ; A / B \quad b ; \boldsymbol{a} ; B}{a \circ b ;\left\{\mathcal{F}_{\mathbf{1}}(\boldsymbol{a}) \ldots \mathcal{F}_{\boldsymbol{n}}(\boldsymbol{a})\right\} ; A}
$$

It can moreover be formally proven that the local and nonlocal modellings of 'respective' predication make exactly the same predictions as to the range of available 'respective' readings and internal readings for symmetrical predicates: in both approaches, it is possible to relate two (or more) terms embedded arbitrarily deeply in different parts of the sentence in the 'respective' manner. This is an interesting result, since one might a priori be inclined to think that the local modelling would be inherently less powerful than the nonlocal modelling. It is of course conceivable to entertain a constrained version of local modelling in which percolation of a multiset structure is blocked in certain syntactic environments (such as islands and complements of certain types of predicates). But similar effects can probably be achieved in the nonlocal modelling as well, by constraining the steps of hypothetical reasoning involved in 'respective' predication in some way or other (in relation to this, see Pogodalla and Pompigne (2012) for an implementation of scope islands in Abstract Categorial Grammar, a framework of CG closely related to Hybrid TLCG).

As noted briefly in relation to the discussion of island constraints in Sect. 2.2.3, choosing between these two alternative approaches on empirical grounds seems to be a complex matter to decide. However, whatever turns out to be the nature of locality constraints on 'respective', symmetrical and summative predicates, we believe that the kind of general setup we have offered in this paper is useful for comparing different hypotheses about them, as it enables one to formulate both the local and nonlocal modelling of 'respective' predication within a single platform. At the very least, the unifying perspective we have offered on these two approaches is interesting in that it relativizes the debate between 'derivational' and 'nonderivational' theories: so far as the semantics of 'respective' predicates is concerned, our analysis shows 
that the extra machinery one needs to introduce in the grammar in each setup is more or less the same, and that the difference in the two types of strategies representative in the two theories is more superficial than real.

\section{A Ancillary derivations}

$$
\begin{aligned}
& \begin{array}{l}
\frac{\left[\varphi_{3} ; R ; \mathrm{VP} / \mathrm{NP}\right]^{3} \quad \text { robin; } \mathbf{r} ; \mathrm{NP}}{\varphi_{3} \circ \text { robin } ; R(\mathbf{r}) ; \mathrm{VP}} / \mathrm{E} \quad \begin{array}{l}
\text { on } \circ \text { thursday; } \\
\text { onTh } ; \mathrm{VP} \backslash \mathrm{VP}
\end{array} \\
\frac{\varphi_{3} \circ \text { robin } \circ \text { on } \circ \text { thursday; } \text { onTh }(R(\mathbf{r})) ; \mathrm{VP}}{\text { robin } \circ \text { on } \circ \text { thursday; } \lambda R . \mathrm{onTh}(R(\mathbf{r})) ;(\mathrm{VP} / \mathrm{NP}) \backslash \mathrm{VP}} \backslash I^{3}
\end{array} \\
& \underline{\left[\varphi_{1} ; x ; \mathrm{NP}\right]^{1}} \frac{\text { met; met; VP } / \mathrm{NP}\left[\varphi_{2} ; \mathscr{P} ;(\mathrm{VP} / \mathrm{NP}) \backslash \mathrm{VP}\right]^{2}}{\operatorname{met} \circ \varphi_{2} ; \mathscr{P}(\text { met }) ; \mathrm{VP}} \backslash \mathrm{E} \\
& \varphi_{1} \circ \text { met } \circ \varphi_{2} ; \mathscr{P}(\text { met })(x) ; \mathrm{S} \\
& \overline{\lambda \varphi_{2} \cdot \varphi_{1} \circ \text { met } \circ \varphi_{2} ; \lambda \mathscr{P} . \mathscr{P}(\text { met })(x) ; \mathrm{S}\lceil((\mathrm{VP} / \mathrm{NP}) \backslash \mathrm{VP})}\left\lceil\mathrm{I}^{2}\right. \\
& \overline{\lambda \varphi_{1} \lambda \varphi_{2} . \varphi_{1} \circ \operatorname{met} \circ \varphi_{2} ; \lambda x \lambda \mathscr{P} . \mathscr{P}(\text { met })(x) ; \mathrm{S} \uparrow((\mathrm{VP} / \mathrm{NP}) \backslash \mathrm{VP})\lceil\mathrm{NP}} \mid \mathrm{I}^{1}
\end{aligned}
$$

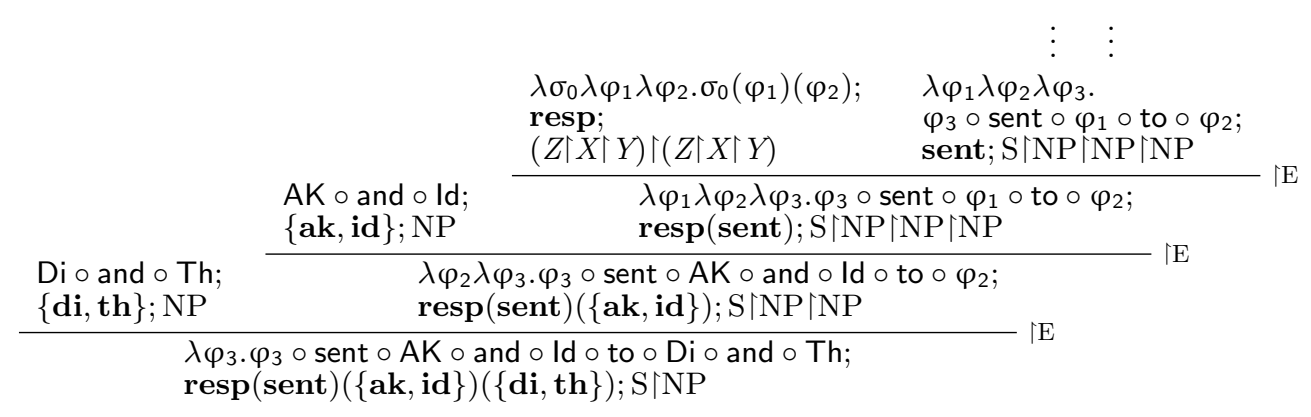

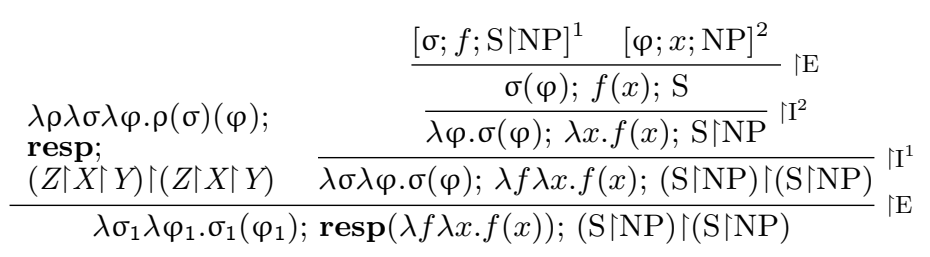

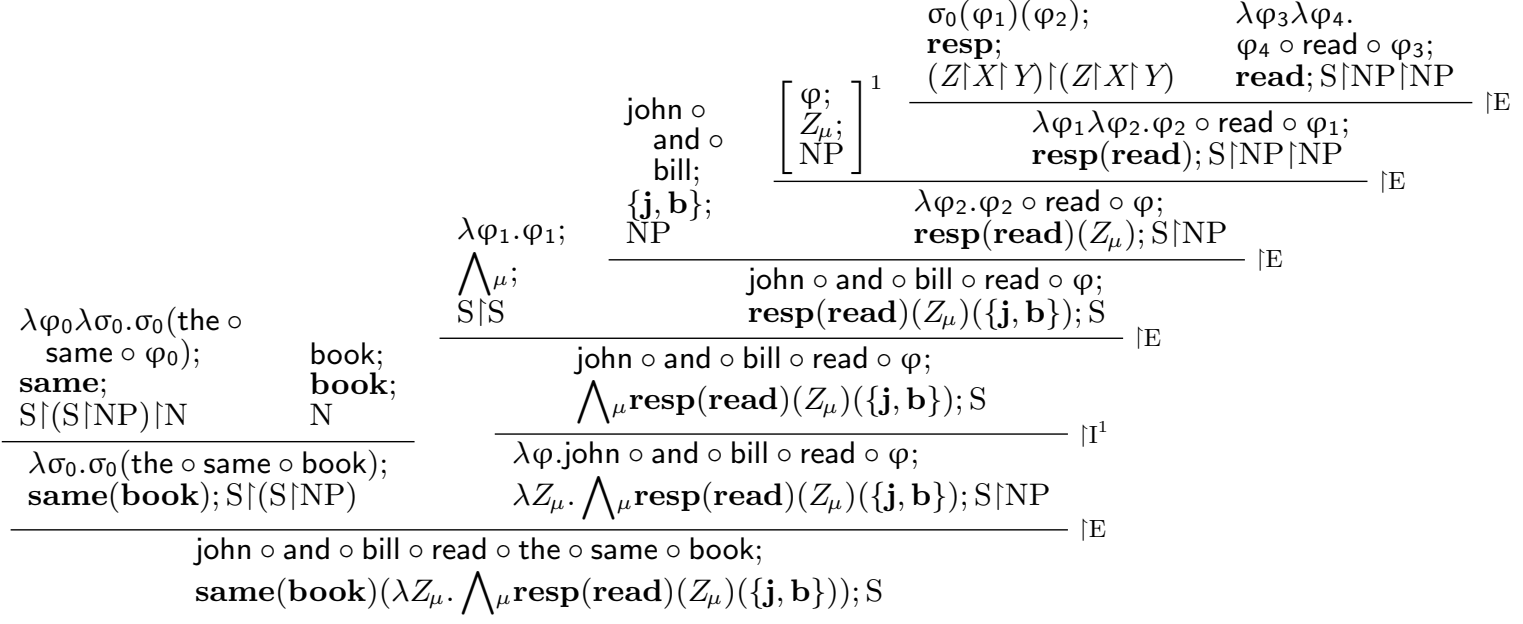

$\lambda \varphi_{0} \lambda \sigma_{0} \cdot \sigma_{0}($ the $\circ$ same $\left.\circ \varphi_{0}\right)$; same; $\mathrm{S}\lceil(\mathrm{S}\lceil\mathrm{NP})\lceil\mathrm{N}$ 
(121)

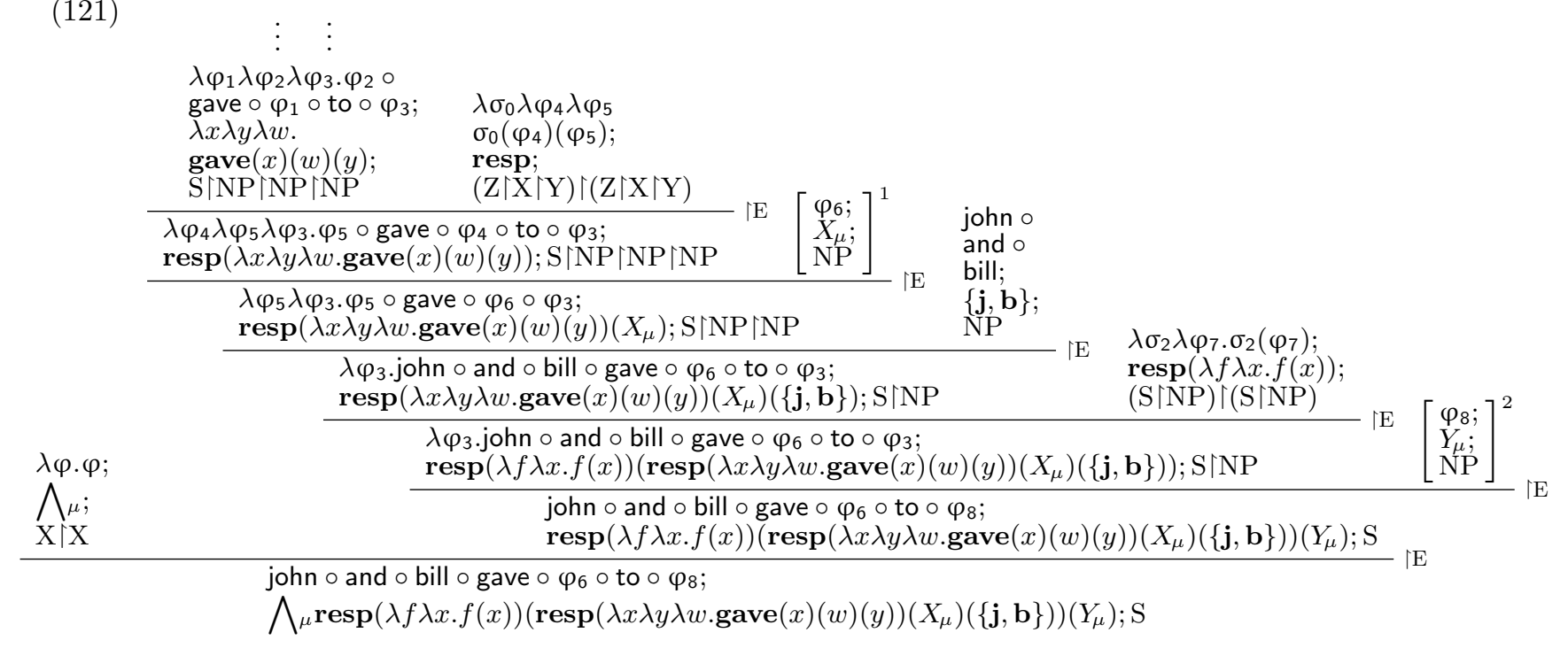
john $\circ$ and $\circ$ bill $\circ$ gave $\circ \varphi_{6} \circ$ to $\circ \varphi_{8}$

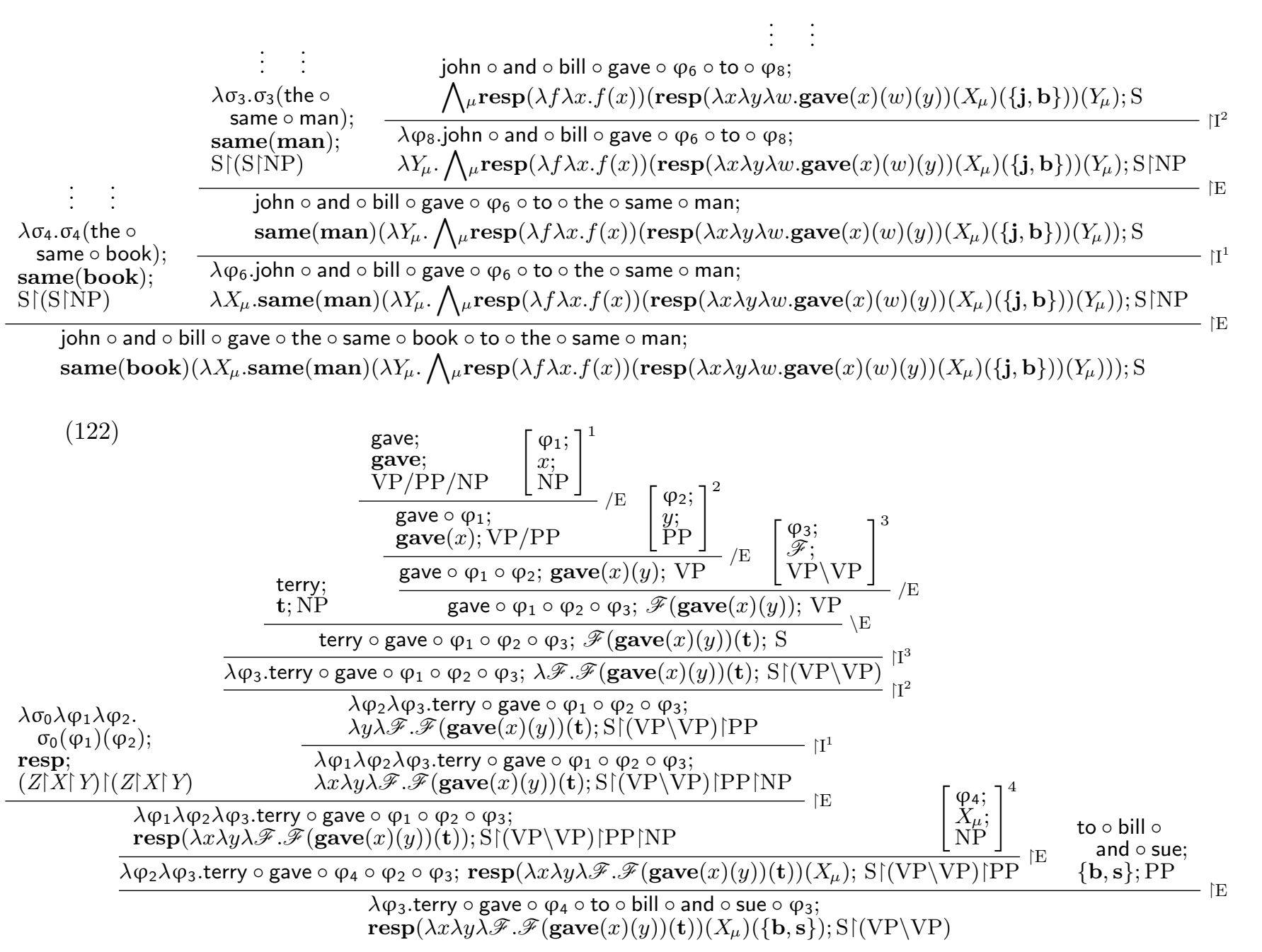




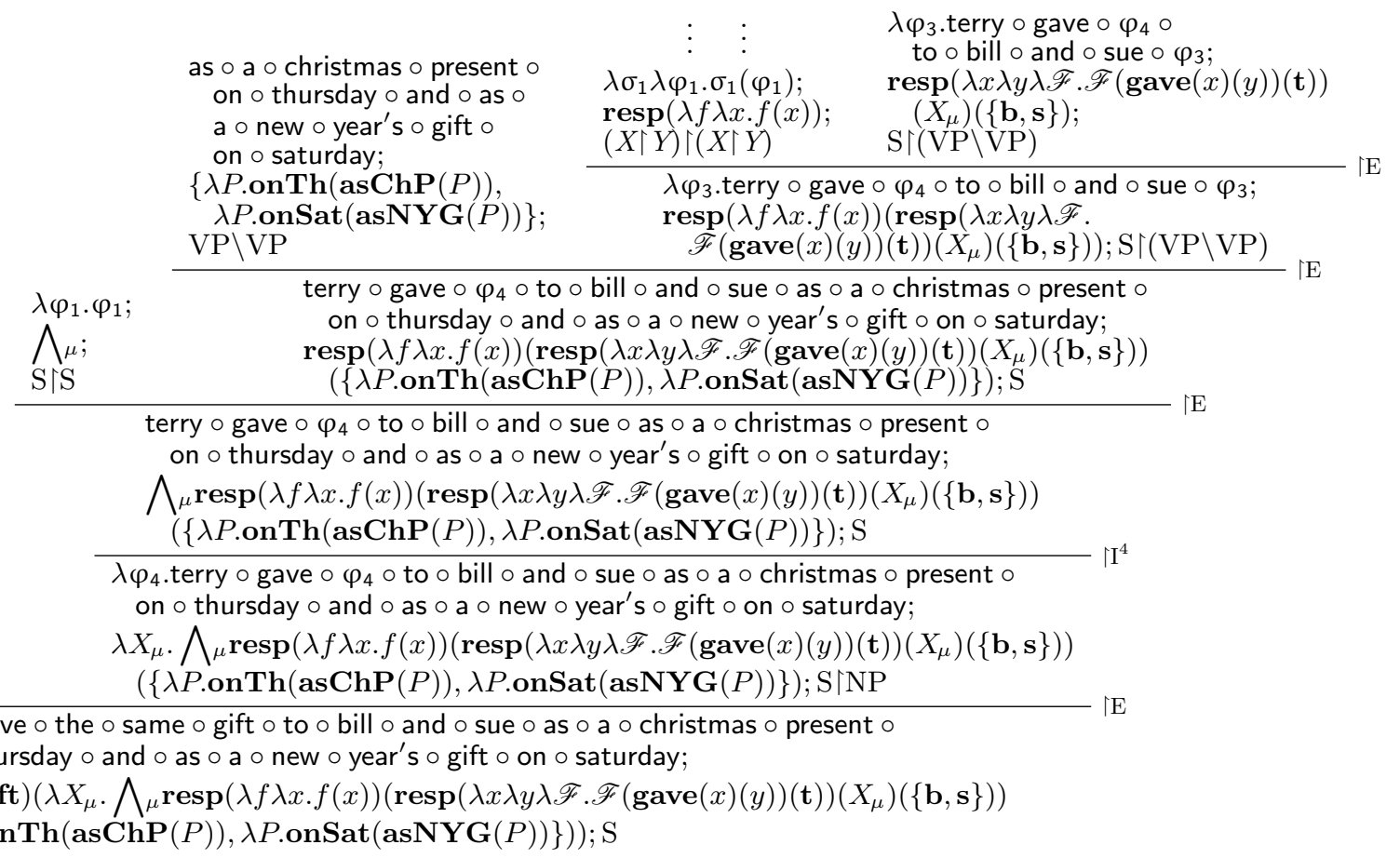

$\begin{array}{ll}\text { the } \circ \text { same } \circ & \text { on } \circ \text { thursday } \circ \text { and } \circ \text { as } \circ \text { a } \circ \text { new } \circ \text { year's } \circ \text { gift } \circ \text { on } \circ \text { saturday; } \\ \text { gift; } & \lambda X_{\mu} . \bigwedge_{\mu} \operatorname{resp}(\lambda f \lambda x . f(x))\left(\operatorname{resp}(\lambda x \lambda y \lambda \mathscr{F} . \mathscr{F}(\operatorname{gave}(x)(y))(\mathbf{t}))\left(X_{\mu}\right)(\{\mathbf{b}, \mathbf{s}\})\right) \\ \text { same }(\text { gift }) ; & (\{\lambda P \text { onTh }(\operatorname{asChP}(P)), \lambda P \text { onSat }(\operatorname{asNYG}(P))\}) ; \mathrm{SPP}\end{array}$

terry $\circ$ gave $\circ$ the $\circ$ same $\circ$ gift $\circ$ to $\circ$ bill $\circ$ and $\circ$ sue $\circ$ as $\circ$ a $\circ$ christmas $\circ$ present $\circ$

on $\circ$ thursday $\circ$ and $\circ$ as $\circ$ a $\circ$ new $\circ$ year's $\circ$ gift $\circ$ on $\circ$ saturday;

$\operatorname{same}($ gift $)\left(\lambda X_{\mu} . \bigwedge \mu \operatorname{resp}(\lambda f \lambda x . f(x))\left(\operatorname{resp}(\lambda x \lambda y \lambda \mathscr{F} . \mathscr{F}(\operatorname{gave}(x)(y))(\mathbf{t}))\left(X_{\mu}\right)(\{\mathbf{b}, \mathbf{s}\})\right)\right.$

$(\{\lambda P . \operatorname{onTh}(\operatorname{asChP}(P)), \lambda P$.onSat $(\operatorname{asNYG}(P))\})) ; \mathrm{S}$

\begin{tabular}{|c|c|}
\hline \multirow{4}{*}{$\begin{array}{l}\lambda \sigma \lambda \varphi_{1} \lambda \varphi_{2} \cdot \sigma\left(\varphi_{1}\right)\left(\varphi_{2}\right) \\
\text { resp; } \\
(Z \uparrow X \uparrow Y) \uparrow(Z\lceil X \uparrow Y)\end{array}$} & {$\left[\varphi_{1} ; f ; \mathrm{S} / \mathrm{NP}\right]^{1} \quad\left[\varphi_{2} ; x ; \mathrm{NP}\right]^{2}$} \\
\hline & 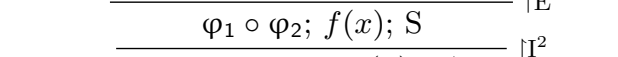 \\
\hline & ${\overline{\lambda \varphi_{2} . \varphi_{1} \circ \varphi_{2} ; \lambda x . f(x) ; \mathrm{S} \backslash \mathrm{NP}}}^{11}$ \\
\hline & $\overline{\lambda \varphi_{1} \lambda \varphi_{2} . \varphi_{1} \circ \varphi_{2} ; \lambda f \lambda x . f(x) ; \mathrm{S}\lceil\mathrm{NP} \uparrow(\mathrm{S} / \mathrm{NP})}$ \\
\hline
\end{tabular}

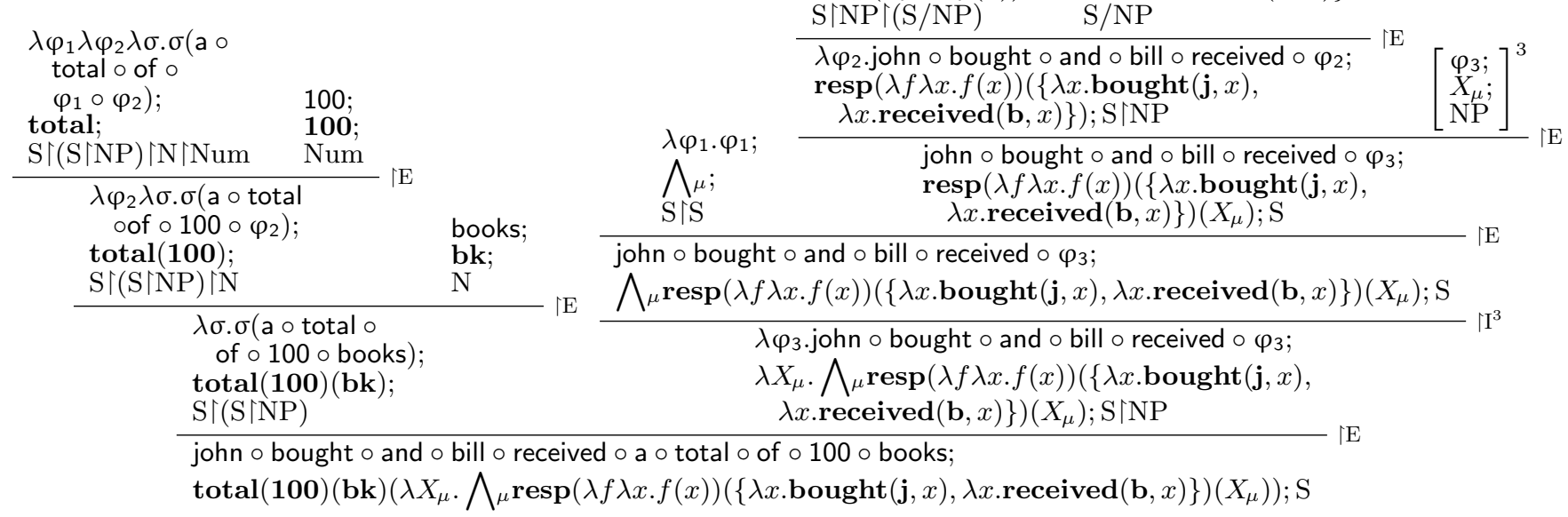




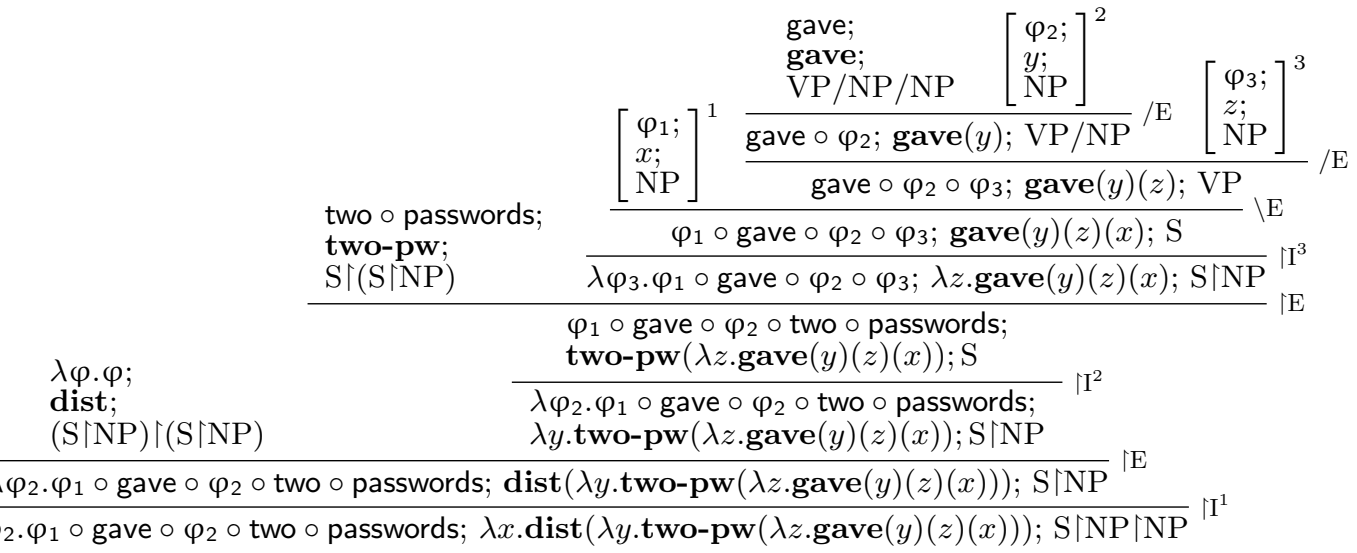

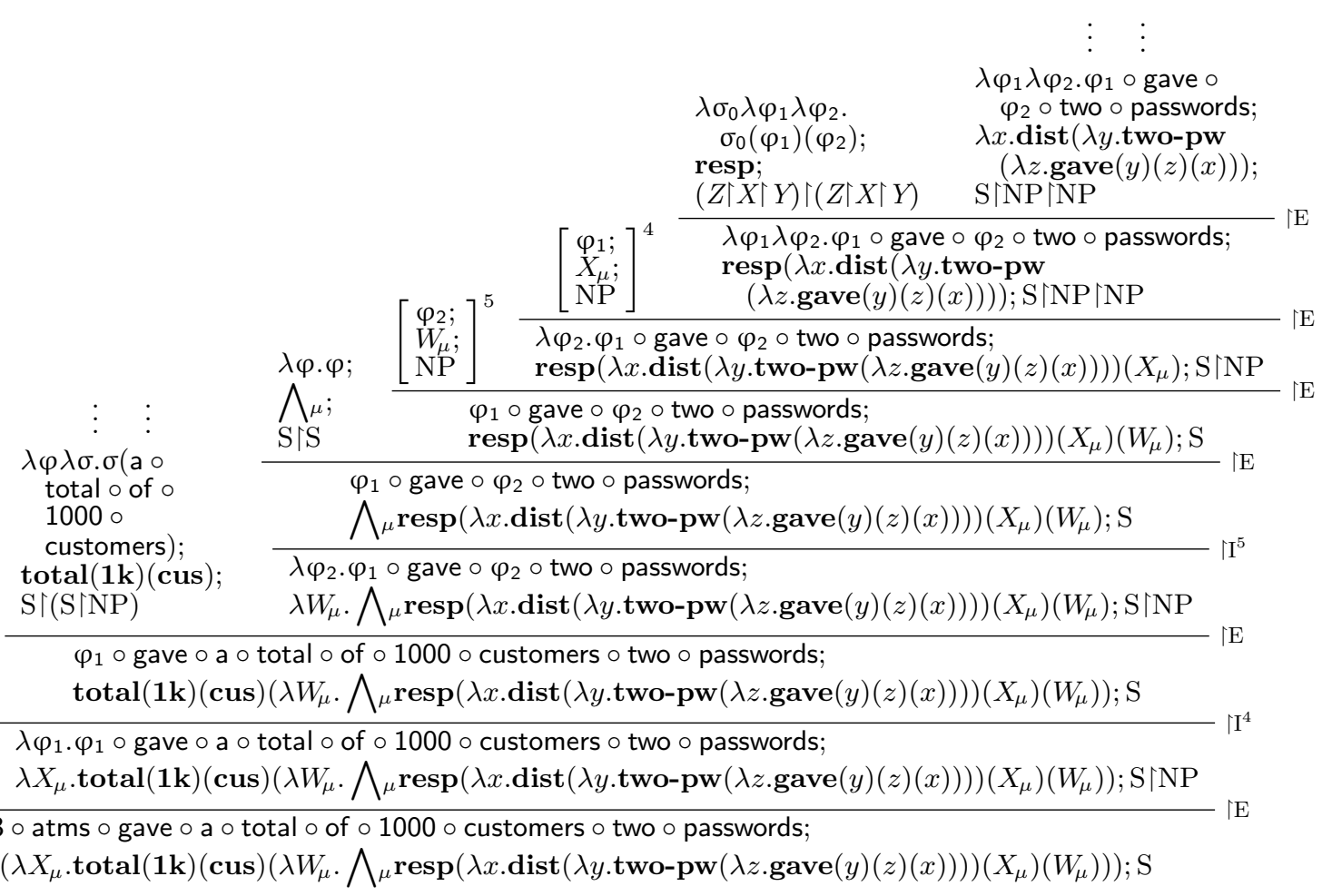

\section{References}

Abbott, Barbara. 1976. Right node raising as a test for constituenthood. Linguistic Inquiry 7:639-642.

Ades, Anthony F. and Mark J. Steedman. 1982. On the order of words. Linguistics and Philosophy 4(4):517-558.

Bach, Emmon. 1986. The algebra of events. Linguistics and Philosophy 9(1):5-16.

Bachrach, Asaf and Roni Katzir. 2007. Spelling out QR. In E. Puig-Waldmueller, ed., Proceedings of Sinn und Bedeutung 11, 63-75. Barcelona: Universitat Pompeu Fabra.

Bachrach, Asaf and Roni Katzir. 2008. Right-node raising and delayed spellout. In K. K. Grohmann, ed., InterPhases: Phase-Theoretic Investigations of Linguistic Interfaces, 249-259. Oxford: OUP.

Barker, Chris. 2007. Parasitic scope. Linguistics and Philosophy 30:407-444.

Barker, Chris. 2012. The same people ordered the same dishes. In T. Graf, D. Paperno, A. Szabolcsi, 
and J. Tellings, eds., UCLA Working Papers in Linguistics: Theories of Everything, vol. 17, 7-14. Department of Linguistics, UCLA.

Barker, Chris. 2013. Scopability and sluicing. Linguistics and Philosophy 36:187-223.

Barker, Chris and Chung-chieh Shan. 2015. Continuations and Natural Language. Oxford: OUP.

Beavers, John and Ivan A. Sag. 2004. Coordinate ellipsis and apparent non-constituent coordination. In S. Müller, ed., The Proceedings of the 11th International Conference on Head-Driven Phrase Structure Grammar, 48-69. Stanford: CSLI.

Beck, Sigrid. 2000. The semantics of 'different': Comparison operator and relational adjective. Linguistics and Philosophy 23(2):101-139.

Bekki, Daisuke. 2006. Heikooteki-kaishaku-niokeru yoosokan-junjo-to bunmyaku-izon-sei (The order of elements and context dependence in the 'respective' interpretation). In Nihon Gengo-Gakkai Dai 132-kai Taikai Yokooshuu (Proceedings from the 132nd Meeting of the Linguistic Society of Japan), $47-52$.

Bernardi, Raffaella. 2002. Reasoning with Polarity in Categorial Type Logic. Ph.D. thesis, University of Utrecht.

Blaheta, Don. 2003. Binominal each: Evidence for a modified type system. Master's thesis, Brown University.

Brasoveanu, Adrian. 2011. Sentence-internal different as quantifier-internal anaphora. Linguistics and Philosophy 34(2):93-168.

Bumford, Dylan and Chris Barker. 2013. Association with distributivity and the problem of multiple antecedents for singular different. Linguistics and Philosophy 36:355-369.

Carlson, Greg N. 1987. Same and different: Some consequences for syntax and semantics. Linguistics and Philosophy 10(4):531-565.

Champollion, Lucas. 2010. Cumulative readings of every do not provide evidence for events and thematic roles. In M. Aloni, H. Bastiaanse, T. de Jager, and K. Schulz, eds., Logic, Language and Meaning, 213-222. Heidelberg: Springer.

Chaves, Rui Pedro. 2008. Linearization-based word-part ellipsis. Linguistics and Philosophy 31(3):261307.

Chaves, Rui Pedro. 2012. Conjunction, cumulation and respectively readings. Journal of Linguistics 48(2):297-344.

Dalrymple, Mary and Andrew Kehler. 1995. On the constraints imposed by respectively. Linguistic Inquiry 26(3):531-536.

Deane, Paul. 1991. Limits to attention: A cognitive theory of island phenomena. Cognitive Linguistics $2(1): 1-63$.

Dotlačil, Jakub. 2010. Anaphora and Distributivity. Ph.D. thesis, Utrecht University.

Dotlačil, Jakub. 2012. Binominal each as an anaphoric determiner: compositional analysis. In A. A. Guevara, A. Chernilovskaya, and R. Nouwen, eds., Proceedings of Sinn und Bedeutung, vol. 16, 211-225. Cambridge, Mass.: MIT Press.

Dowty, David. 1985. A unified indexical analysis of same and different: A response to Stump and Carlson. MS., Ohio State University.

Dowty, David R. 1996. Toward a minimalist theory of syntactic structure. In H. Bunt and A. van Horck, eds., Discontinuous Constituency, vol. 6 of Natural Language Processing, 11-62. Berlin, New York: Mouton de Gruyter.

Gawron, Jean Mark and Andrew Kehler. 2002. The semantics of the adjective respective. In Proceedings of WCCFL 21, 85-98.

Gawron, Jean Mark and Andrew Kehler. 2004. The semantics of respective readings, conjunction, and filler-gap dependencies. Linguistics and Philosophy 27(2):169-207.

Heim, Irene. 1985. Notes on comparatives and related matters. MS, University of Texas, Austin.

Hendriks, Petra. 1995. Comparatives and Categorial Grammar. Ph.D. thesis, University of Groningen. 
Hofmeister, Philip. 2010. A linearization account of either ... or constructions. Natural Language and Linguistic Theory 28:275-314.

Hofmeister, Philip and Ivan A. Sag. 2010. Cognitive constraints and island effects. Language 86(2):366415.

Hudson, Richard A. 1976. Conjunction reduction, gapping and right-node raising. Language $52(3): 535-562$.

Jackendoff, Ray. 1977. X-bar Syntax: A Study of Phrase Structure. Cambridge, Mass.: MIT Press.

Kay, Paul. 1989. Contextual operators: respective, respectively, and vice versa. In Proceedings of BLS 15, 181-192. Berkeley, CA.

Keenan, Edward L. 1992. Beyond the Frege boundary. Linguistics and Philosophy 15(2):199-221.

Kehler, Andrew. 2002. Coherence, Reference and the Theory of Grammar. Stanford, California: CSLI Publications.

Kennedy, Christopher and Jason Stanley. 2008. What an average semantics needs. In T. Friedman and S. Ito, eds., Proceedings of SALT 18, 465-482. Ithaca, New York: Cornell University.

Kluender, Robert. 1992. Deriving island constraints from principles of predication. In H. Goodluck and M. Rochemont, eds., Island Constraints: Theory, Acquisition, and Processing, 223-258. Dordrecht: Kluwer.

Kluender, Robert. 1998. On the distinction between strong and weak islands: A processing perspective. In P. Culicover and L. McNally, eds., The Limits of Syntax, vol. 29 of Syntax and Semantics, 241279. San Diego: Academic Press.

Kratzer, Angelika. 2007. On the plurality of verbs. In J. Dölling, T. Heyde-Zybatow, and M. Schäfer, eds., Event Structures in Linguistic Form and Interpretation, 269-300. Mouton de Gruyter.

Krifka, Manfred. 1989. Nominal reference, temporal constitution, and quantification in event semantics. In R. Bartsch, J. van Benthem, and P. van Emde Boas, eds., Semantics and Contextual Expression, 75-115. Dordrecht: Foris Publications.

Krifka, Manfred. 1990. Boolean and non-boolean 'and'. In L. Kálman and L. Polos, eds., Papers from the Second Symposium on Logic and Language, 161-188. Budapest: Akadémiai Kiadó.

Krifka, Manfred. 1992. Thematic relations as links between nominal reference and temporal constitution. In I. Sag and A. Szabolski, eds., Lexical Matters, 30-52. University of Chicago Press.

Kubota, Yusuke. 2010. (In)flexibility of Constituency in Japanese in Multi-Modal Categorial Grammar with Structured Phonology. Ph.D. thesis, Ohio State University.

Kubota, Yusuke. 2014. The logic of complex predicates: A deductive synthesis of 'argument sharing' and 'verb raising'. Natural Language and Linguistic Theory 32(4):1145-1204.

Kubota, Yusuke. 2015. Nonconstituent coordination in Japanese as constituent coordination: An analysis in Hybrid Type-Logical Categorial Grammar. Linguistic Inquiry 46(1):1-42.

Kubota, Yusuke and Robert Levine. 2013a. Coordination in Hybrid Type-Logical Categorial Grammar. In OSU Working Papers in Linguistics, vol. 60, 21-50. Department of Linguistics, Ohio State University.

Kubota, Yusuke and Robert Levine. 2013b. Determiner gapping as higher-order discontinuous constituency. In G. Morrill and M.-J. Nederhof, eds., Proceedings of Formal Grammar 2012 and 2013, 225-241. Heidelberg: Springer.

Kubota, Yusuke and Robert Levine. 2014a. Gapping as hypothetical reasoning. To appear in NLLT, available at http://ling. auf .net/lingbuzz/002123.

Kubota, Yusuke and Robert Levine. 2014b. Hybrid Type-Logical Categorial Grammar. MS., University of Tsukuba and Ohio State University, available at http://ling. auf .net/lingbuzz/002313.

Kubota, Yusuke and Robert Levine. 2014c. Pseudogapping as pseudo-VP ellipsis. In N. Asher and S. Soloviev, eds., Logical Aspects of Computational Linguistics 2014, 122-137. Heidelberg: Springer.

Kubota, Yusuke and Robert Levine. 2014d. Unifying local and nonlocal modelling of respective and symmetrical predicates. In G. Morrill, R. Muskens, R. Osswald, and F. Richter, eds., Proceedings of Formal Grammar 2014, 104-120. Heidelberg: Springer. 
Kubota, Yusuke and Robert Levine. 2015. Against ellipsis: Arguments for the direct licensing of 'noncanonical' coordinations. To appear in Linguistics and Philosophy, MS., University of Tsukuba and Ohio State University, available at http://ling. auf .net/lingbuzz/002214.

Lambek, Joachim. 1958. The mathematics of sentence structure. American Mathematical Monthly 65:154-170.

Landman, Fred. 2000. Events and Plurality. Dordrecht: Kluwer.

Lasersohn, Peter. 1988. A Semantics for Groups and Events. Ph.D. thesis, Ohio State University.

Lasersohn, Peter. 1992. Generalized conjunction and temporal modification. Linguistics and Philosophy 15(4):381-410.

Matthewson, Lisa. 2001. Quantification and the nature of crosslinguistic variation. Natural Language Semantics 9(2):145-189.

McCawley, James D. 1998. The Syntactic Phenomena of English. Chicago: University of Chicago Press, 2nd edn.

Mihaliček, Vedrana and Carl Pollard. 2012. Distinguishing phenogrammar from tectogrammar simplifies the analysis of interrogatives. In P. de Groote and M.-J. Nederhof, eds., Formal Grammar 2010/2011, 130-145. Heidelberg: Springer.

Moltmann, Frederike. 1992. Reciprocals and same/different: Towards a semantic analysis. Linguistics and Philosophy 15(4):411-462.

Montague, Richard. 1973. The proper treatment of quantification in ordinary English. In J. Hintikka, J. M. Moravcsik, and P. Suppes, eds., Approaches to Natural Language: Proceedings of the 1970 Stanford Workshop on Grammar and Semantics, 221-242. Dordrecht, Holland: D. Reidel.

Moortgat, Michael. 1997. Categorial Type Logics. In J. van Benthem and A. ter Meulen, eds., Handbook of Logic and Language, 93-177. Amsterdam: Elsevier.

Moortgat, Michael and Richard T. Oehrle. 1994. Adjacency, dependence, and order. In P. Dekker and M. Stokhof, eds., Proceedings of the Ninth Amsterdam Colloquium, 447-466. Universiteit van Amsterdam: Instituut voor Taal, Logica, en Informatica.

Moot, Richard. 2014. Hybrid type-logical grammars, first-order linear logic and the descriptive inadequacy of Lambda grammars. MS., Laboratoire Bordelais de Recherche en Informatique.

Morrill, Glyn. 1994. Type Logical Grammar: Categorial Logic of Signs. Dordrecht: Kluwer.

Morrill, Glyn. 2010. Categorial Grammar: Logical Syntax, Semantics, and Processing. Oxford: OUP.

Morrill, Glyn and Teresa Solias. 1993. Tuples, discontinuity, and gapping in categorial grammar. In Proceedings of the Sixth Conference on European Chapter of the Association for Computational Linguistics, 287-296. Morristown, NJ, USA: Association for Computational Linguistics.

Morrill, Glyn, Oriol Valentín, and Mario Fadda. 2011. The displacement calculus. Journal of Logic, Language and Information 20:1-48.

Mouret, Francois. 2006. A phrase structure approach to argument cluster coordination. In S. Müller, ed., The Proceedings of the 13th International Conference on Head-Driven Phrase Structure Grammar, 247-267. Stanford: CSLI Publications.

Muskens, Reinhard. 2003. Language, lambdas, and logic. In G.-J. Kruijff and R. Oehrle, eds., Resource Sensitivity in Binding and Anaphora, 23-54. Dordrecht: Kluwer.

Muskens, Reinhard. 2007. Separating syntax and combinatorics in categorial grammar. Research on Language and Computation 5(3):267-285.

Oehrle, Richard. 1996. Austinian pluralities. In J. Seligman and D. Westerståhl, eds., Language, logic, and computation, 433-441. Stanford: CSLI Publications.

Oehrle, Richard T. 1994. Term-labeled categorial type systems. Linguistics and Philosophy 17(6):633678.

Okada, Sadayuki. 1999. On the function and distribution of the modifiers respective and respectively. Linguistics 37(5):871-903.

Partee, Barbara and Mats Rooth. 1983. Generalized conjunction and type ambiguity. In R. Bäuerle, C. Schwarze, and A. von Stechow, eds., Meaning, Use, and Interpretation of Language, 361-383. 
Berlin: Walter de Gruyter.

Phillips, Colin. 1996. Order and structure. Ph.D. thesis, MIT.

Pogodalla, Sylvain and Florent Pompigne. 2012. Controlling extraction in Abstract Categorial Grammars. In P. de Groote and M.-J. Nederhof, eds., Formal Grammar 2010/2011, 162-177. Heidelberg: Springer.

Pollard, Carl. 2014. What numerical determiners mean: A non-ambiguity analysis. Talk presented at the Workshop on Semantics of Cardinals, Ohio State University, March 6, 2014.

Pollard, Carl and E. Allyn Smith. 2012. A unified analysis of the same, phrasal comparatives and superlatives. In Proceedings of SALT 2012, 307-325.

Postal, Paul M. 1998. Three Investigations of Extraction. Cambridge, Mass.: MIT Press.

Pullum, Geoffrey and Gerald Gazdar. 1982. Natural languages and context-free languages. Linguistics and Philosophy 4(4):471-504.

Ruys, Eddy and Yoad Winter. 2010. Quantifier scope in formal linguistics. In D. Gabbay and F. Guenthner, eds., Handbook of Philosophical Logic, vol. 16, 159-225. Amsterdam: John Benjamins.

Scha, Remko. 1981. Distributive, collective and cumultative quantification. In J. Groenendijk, T. Janssen, and M. Stokhof, eds., Formal Methods in the Study of Language, 483-512. Amsterdam: Universiteit Amsterdam, Mathematical Center.

Schein, Barry. 1993. Plurals and events. Cambridge, Mass.: MIT Press.

Schmitt, Viola. 2013. More Pluralities. Ph.D. thesis, Universität Wien.

Schwarzschild, Roger. 1996. Pluralities. Dordrecht: Kluwer.

Steedman, Mark. 1985. Dependency and coordination in the grammar of Dutch and English. Language 61(3):523-568.

Steedman, Mark. 1996. Surface Structure and Interpretation. Cambridge, Mass.: MIT Press.

Steedman, Mark. 2000. The Syntactic Process. Cambridge, Mass.: MIT Press.

Steedman, Mark. 2012. Taking Scope. Cambridge, Mass.: MIT Press.

Szabolcsi, Anna. 1997. Strategies for scope taking. In A. Szabolcsi, ed., Ways of Scope Taking, 109-154. Dordrecht: Kluwer.

Vermaat, Willemijn. 2005. The Logic of Variation. A Cross-Linguistic Account of Wh-Question Formation. Ph.D. thesis, Utrecht University.

Winter, Yoad. 1995. Syncategorematic conjunction and structured meanings. In M. Simons and T. Galloway, eds., Proceedings of SALT 5, 387-404. Ithaca, NY: CLC Publications. 